\title{
AN ECONOMETRIC PERSPECTIVE ON ALGORITHMIC SUBSAMPLING
}

\author{
Sokbae LeE* SEREnA $\mathrm{NG}^{\dagger}$
}

May 1, 2020

\begin{abstract}
Datasets that are terabytes in size are increasingly common, but computer bottlenecks often frustrate a complete analysis of the data. While more data are better than less, diminishing returns suggest that we may not need terabytes of data to estimate a parameter or test a hypothesis. But which rows of data should we analyze, and might an arbitrary subset of rows preserve the features of the original data? This paper reviews a line of work that is grounded in theoretical computer science and numerical linear algebra, and which finds that an algorithmically desirable sketch, which is a randomly chosen subset of the data, must preserve the eigenstructure of the data, a property known as a subspace embedding. Building on this work, we study how prediction and inference can be affected by data sketching within a linear regression setup. We show that the sketching error is small compared to the sample size effect which a researcher can control. As a sketch size that is algorithmically optimal may not be suitable for prediction and inference, we use statistical arguments to provide 'inference conscious' guides to the sketch size. When appropriately implemented, an estimator that pools over different sketches can be nearly as efficient as the infeasible one using the full sample.
\end{abstract}

Keywords: sketching, coresets, subspace embedding, countsketch, uniform sampling. JEL Classification: C2, C3.

\footnotetext{
${ }^{*}$ Department of Economics, Columbia University and Institute for Fiscal Studies

${ }^{\dagger}$ Department of Economics, Columbia University and NBER

The authors would like to thank David Woodruff and Shusen Wang for helpful discussions. The first author would like to thank the European Research Council for financial support (ERC-2014-CoG- 646917-ROMIA) and the UK Economic and Social Research Council for research grant (ES/P008909/1) to the CeMMAP. The second author would like to thank the National Science Foundation for financial support (SES: 1558623).
} 


\section{Introduction}

The availability of terabytes of data for economic analysis is increasingly common. But analyzing large datasets is time consuming and sometimes beyond the limits of our computers. The need to work around the data bottlenecks was no smaller decades ago when the data were in megabytes than it is today when data are in terabytes and petabytes. One way to alleviate the bottleneck is to work with a sketch of the data 1 These are data sets of smaller dimensions and yet representative of the original data. We study how the design of linear sketches affects estimation and inference in the context of the linear regression model. Our formal statistical analysis complements those in the theoretical computer science and numerical linear algebra derived using different notions of accuracy and whose focus is computation efficiency.

There are several motivations for forming sketches of the data from the full sample. If the data are too expensive to store and/or too large to fit into computer memory, the data would be of limited practical use. It might be cost effective in some cases to get a sense from a smaller sample whether an expensive test based on the full sample is worth proceeding. Debugging is certainly faster with fewer observations. A smaller dataset can be adequate while a researcher is learning how to specify the regression model, as loading a gigabyte of data is much faster than a terabyte even we have enough computer memory to do so. With confidentiality reasons, one might only want to circulate a subset rather than the full set of data.

For a sketch of the data to be useful, the sketch must preserve the characteristics of the original data. Early work in the statistics literature used a sketching method known as 'data squashing'. The idea is to approximate the likelihood function by merging data points with similar likelihood profiles. There are two different ways to squash the data. One approach is to construct subsamples randomly. While these methods work well for the application under investigation, its general properties are not well understood. An alternative is to take the data structure into account. Du Mouchel et al. (1999) also forms multivariate bins of the data, but they match low order moments within the bin by non-linear optimization. Owen (1990) reweighs a random sample of $X$ to fit the moments using empirical likelihood estimation. Madigan et al. (1999) uses likelihood-based clustering to select data points that match the target distribution. While theoretically appealing, modeling the likelihood profiles can itself be time consuming and not easily scalable.

Data sketching is of also interest to computer scientists because they are frequently required to provide summaries (such as frequency, mean, and maximum) of data as they stream by continuously ${ }_{2}^{2}$ Instead of an exact answer which would be costly to compute, pass-efficient randomized

\footnotetext{
${ }^{1}$ The term 'synopsis' and 'coresets' have also been used. See Comrode et al. (2011), and Agarwal and Varadarajan (2004). We generically refer to these as sketches.

${ }^{2}$ The seminal paper on frequency moments is Alon et al. (1999). For a review of the literature, he reader is referred
} 
algorithms are designed to run fast, requires little storage, and guarantee the correct answer with a certain probability. But this is precisely the underlying premise of data sketching in statistical analysis ${ }^{3}$

Though randomized algorithms are increasingly used for sketching in a wide range of applications, the concept remains largely unknown to economists except for a brief exposition in $\mathrm{Ng}$ (2017). This paper provides a gentle introduction to these algorithms in Sections 2 to 4 . To our knowledge, this is the first review on sketching in the econometrics literature. We will use the term algorithmic subsampling to refer to randomized algorithms designed for the purpose of sketching, to distinguish them from bootstrap and subsampling methods developed for frequentist inference. In repeated sampling, we only observe one sample drawn from the population. Here, the complete data can be thought of as the population which we observe, but we can only use a subsample. Algorithmic subsampling does not make distributional assumptions, and balancing between fast computation and favorable worst case approximation error often leads to algorithms that are oblivious to the properties the data. In contrast, exploiting the probabilistic structure is often an important aspect of econometric modeling.

Perhaps the most important tension between the algorithmic and the statistical view is that while fast and efficient computation tend to favor sketches with few rows, efficient estimation and inference inevitably favor using as many rows as possible. Sampling schemes that are optimal from an algorithmic perspective may not be desirable from an econometric perspective, but it is entirely possible for schemes that are not algorithmically optimal to be statistically desirable. As there are many open questions about the usefulness of these sampling schemes for statistical modeling, there is an increased interest in these methods within the statistics community. Recent surveys on sketching with a regression focus include Ahfock et al. (2017) and Geppert, Ickstadt, Munteanu, Quedenfeld and Sohler (2017), among others. Each paper offers distinct insights, and the present paper is no exception.

Our focus is on efficiency of the estimates for prediction and inference within the context of the linear regression model. Analytical and practical considerations confine our focus eventually back to uniform sampling, and to a smaller extent, an algorithm known as the countsketch. The results in Sections 5 and 6 are new. It will be shown that data sketching has two effects on estimation, one due to sample size, and one due to approximation error, with the former dominating the later in all quantities of empirical interest. Though the sample size effect has direct implications for the power of any statistical test, it is at the discretion of a researcher. We show that moment restrictions to Comrode et al. (2011).

'Pass-efficient algorithms read in data at most a constant number of times. A computational method is referred to as a streaming model if only one pass is needed. 
can be used to guide the sketch size, with fewer rows being needed when more moments exist. By targeting the power of a test at a prespecified alternative, the size of the sketch can also be tuned so as not to incur excessive power loss in hypothesis testing. We refer to this as the 'inference conscious' sketch size.

There is an inevitable trade-off between computation cost and statistical efficiency, but the statistical loss from using fewer rows of data can be alleviated by combining estimates from different sketches. By the principle of 'divide and conquer', running several estimators in parallel can be statistically efficient and still computationally inexpensive. Both uniform sampling and the countsketch are amenable to parallel processing which facilitates averaging of quantities computed from different sketches. We assess two ways of combining estimators: one that averages the parameter estimates, and one that averages test statistics. Regardless of how information from the different sketches are combined, pooling over subsamples always provides more efficient estimates and more powerful tests. It is in fact possible to bring the power of a test arbitrarily close to the one using the full sample, as will be illustrated in Section 6 .

\subsection{Motivating Examples}

The sketching problem can be summarized as follows. Given an original matrix $A \in \mathbb{R}^{n \times d}$, we are interested in $\widetilde{A} \in \mathbb{R}^{m \times d}$ constructed as

$$
\widetilde{A}=\Pi A
$$

where $\Pi \in \mathbb{R}^{m \times n}, m<n$. In a linear regression setting, $A=[y \quad X]$ where $y$ is the dependent variable, and $X$ indicates the regressors. Computation of the least squares estimator takes $O\left(n d^{2}\right)$ time which becomes costly when the number of rows, $n$ is large. Non-parametric regressions fit into this setup if $X$ is a matrix of sieve basis. Interest therefore arises to use fewer rows of $A$ without sacrificing too much information.

To motivate why the choice of the sampling scheme (ie. П) matters, consider as an example a $5 \times 2$ matrix

$$
A=\left(\begin{array}{ccccc}
1 & 0 & -.25 & .25 & 0 \\
0 & 1 & .5 & -.5 & 0
\end{array}\right)^{T}
$$

The rows have different information content as the row norm is $(1,1,0.559,0.559,0)^{T}$. Consider 
now three $2 \times 2 \widetilde{A}$ matrices constructed as follows:

$$
\begin{aligned}
\Pi_{1}=\left(\begin{array}{lllll}
1 & 0 & 0 & 0 & 0 \\
0 & 1 & 0 & 0 & 0
\end{array}\right), & \widetilde{A}_{1}=\Pi_{1} A=\left(\begin{array}{ll}
1 & 0 \\
0 & 1
\end{array}\right) \\
\Pi_{2}=\left(\begin{array}{lllll}
1 & 0 & 0 & 0 & 0 \\
0 & 0 & 0 & 0 & 1
\end{array}\right), & \widetilde{A}_{2}=\Pi_{2} A=\left(\begin{array}{ll}
1 & 0 \\
0 & 0
\end{array}\right) \\
\Pi_{3}=\left(\begin{array}{ccccc}
0 & 0 & 1 & 1 & 0 \\
0 & 1 & -1 & 1 & 1
\end{array}\right), & \widetilde{A}_{3}=\Pi_{3} A=\left(\begin{array}{cc}
0 & 0 \\
.5 & 0
\end{array}\right) .
\end{aligned}
$$

Of the three sketches, only $\Pi_{1}$ preserves the rank of $A$. The sketch defined by $\Pi_{2}$ fails because it chooses row 5 which has no information. The third sketch is obtained by taking a linear combination of rows that do not have independent information. The point is that unless $\Pi$ is chosen appropriately, $\widetilde{A}$ may not have the same rank as $A$.

Of course, when $m$ is large, changing rank is much less likely and one may also wonder if this pen and pencil problem can ever arise in practice. Consider now estimation of a Mincer equation which has the logarithm of WAGE as the dependent variable, estimated using the IPUMS (2020) dataset which provides a preliminary but complete count data for the 1940 U.S. Census. This data is of interest because it was the first census with information on wages and salary income. For illustration, we use a sample of $n=24$ million white men between the age of 16 and 64 as the 'full sample'. The predictors that can be considered are years of education, denoted (EDU), and potential experience, denoted (EXP).

Figure 1: Distribution of Potential Experience

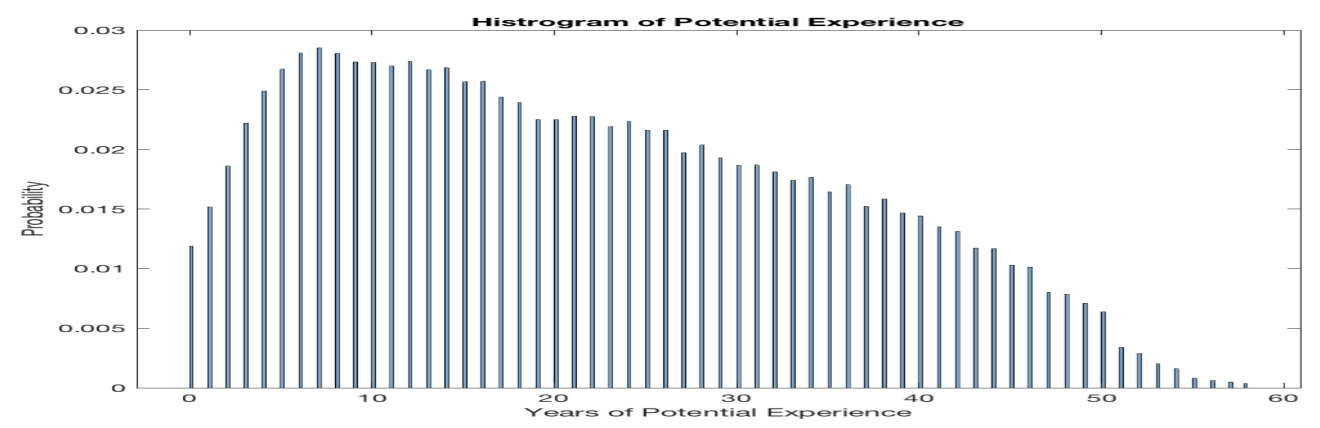

Two Mincer equations with different covariates are considered:

$$
\begin{aligned}
& \log \text { wage }=\beta_{0}+\beta_{1} \mathrm{edu}+\beta_{2} \exp +\beta_{3} \exp ^{2}+\text { error } \\
& \text { log wage }=\beta_{0}+\beta_{1} \mathrm{edu}+\sum_{j=0}^{11} \beta_{2+j} 1\{5 j \leq \exp <5(j+1)\}+\text { error. }
\end{aligned}
$$


Model (1a) uses ExP and $\operatorname{ExP}^{2}$ as control variables. Model $1 \mathrm{~b}$ replaces potential experience with indicators of experience in five year intervals. Even though there are three predictors including the intercept, the number of covariates $K$ is 4 in the first model and 14 in the second. In both cases, the parameter of interest is the coefficient for years of education $\left(\beta_{1}\right)$. The full sample estimate of $\beta_{1}$ is 0.12145 in specification $1 \mathrm{a}$ and 0.12401 in specification $1 \mathrm{~b}$.

Figure 1 shows the histogram of ExP. The values of EXP range from 0 to 58 . The problem in this example arises because there are few observations with over 50 years of experience. Hence there is no guarantee that an arbitrary subsample will include observations with EXP $>50$. Without such observations, the subsampled covariate matrix may not have full rank. Specification $(1 \mathrm{~b}$ ) is more vulnerable to this problem especially when $m$ is small.

We verify that rank failure is empirically plausible in a small experiment with sketches of size $m=100$ extracted using two sampling schemes. The first method is uniform sampling without replacement which is commonly used in economic applications. The second is the countsketch which will be further explained below. Figure 2 and Figure 3 show the histograms of subsample estimates for uniform sampling and the countsketch, respectively. The left panel is for specification (1a) and the right panel is for specification $(1 \mathrm{~b})$. In our experiments, singular matrices never occurred with specification (1a); the OLS estimates can be computed using both sampling algorithms and both performed pretty well. However, uniform sampling without replacement produced singular matrices for specification (1b) $77 \%$ of the time. The estimates seem quite different from the full sample estimates, suggesting not only bias in the estimates, but also that the bias might not be random. In contrast, the countsketch failed only once out of 100 replications. The estimates are shown in the right panel of Figure 3 excluding the singular case.

This phenomenon can be replicated in a Monte Carlo experiment with $K=3$ normally distributed predictors. Instead of $X_{3}$, it is assumed that we only observe a value of one if $X_{3}$ is three standard deviation from the mean. Together with an intercept, there are four regressors. As in the Mincer equation, the regressor matrix has a reduced rank of 3 with probability of $0.58,0.25,0.076$ when $m=200,500,1000$ rows are sampled uniformly; it is always full rank only when $m=2000$. In contrast, the countsketch never encounters this problem even with $m=100$. The simple example underscores the point that the choice of sampling scheme matters. As will be seen below, the issue remains in a more elaborate regression with several hundred covariates. This motivates the need to better understand how to form sketches for estimation and inference. 
Figure 2: Distributions of Estimates from Uniform Sampling without Replacement
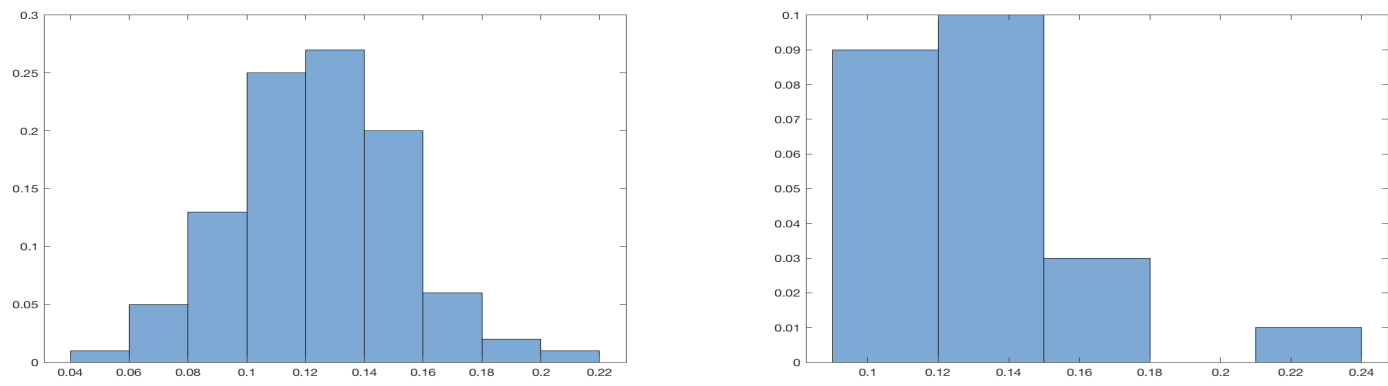

Figure 3: Distributions of Estimates from CountSketch Sampling
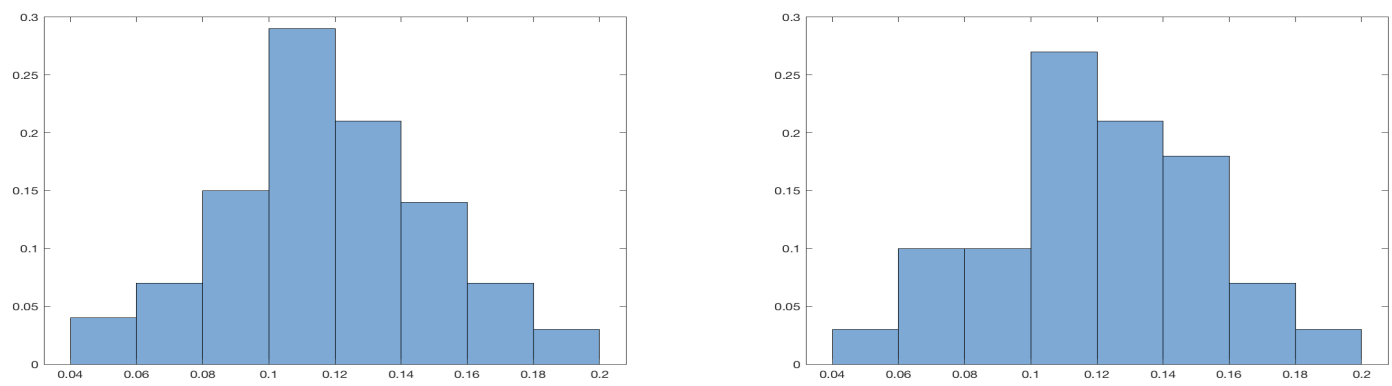

\section{$2 \quad$ Matrix Sketching}

This section presents the key concepts in algorithmic sampling. The material is based heavily on the monographs by Mahoney (2011) and Woodruff (2014), as well as the seminal work of Drineas, Mahoney and Muthukrishnan (2006), and subsequent refinements developed in Drineas et al. (2011), Nelson and Nguyen (2013a), Nelson and Nguyen (2014), Cohen, Nelson and Woodruff (2015), Wang, Gittens and Mahoney (2018), among many others.

We begin by setting up the notation. Consider an $n \times d$ matrix positive-definite $A$. Let $A^{(j)}$ denote its $j$-th column of $A$ and $A_{(i)}$ be its $i$-th row. Then

$$
A=\left(\begin{array}{c}
A_{(1)} \\
\vdots \\
A_{(n)}
\end{array}\right)=\left(\begin{array}{lll}
A^{(1)} & \ldots & A^{(d)}
\end{array}\right)
$$

and $A^{T} A=\sum_{i=1}^{n} A_{(i)}^{T} A_{(i)}$. The singular value decomposition of $A$ is $A=U \Sigma V^{T}$ where $U$ and $V$ are the left and right eigenvectors of dimensions $(n \times d)$ and $(d \times d)$ respectively. The matrix $\Sigma$ is $d \times d$ diagonal with entries containing the singular values of $A$ denoted $\sigma_{1}, \ldots, \sigma_{d}$, which are ordered such that $\sigma_{1}$ is the largest. Since $A^{T} A$ is positive definite, its $k$-th eigenvalue $\omega_{k}\left(A^{T} A\right)$ 
equals $\sigma_{k}\left(A^{T} A\right)=\sigma_{k}^{2}(A)$, for $k=1, \ldots d$. The best rank $k$ approximation of $A$ is given by

$$
A_{k}=U_{k} U_{k}^{T} A \equiv P_{U_{k}} A
$$

where $U_{k}$ is an $n \times k$ orthonormal matrix of left singular vectors corresponding to the $k$ largest singular values of $A$, and $P_{U_{k}}=U_{k} U_{k}^{T}$ is the projection matrix.

The Frobenius norm (an average type criterion) is $\|A\|_{F}=\sqrt{\sum_{i=1}^{n} \sum_{j=1}^{d}\left|A_{i j}\right|^{2}}=\sqrt{\sum_{i=1}^{k} \sigma_{i}^{2}}$. The spectral norm (a worse-case type criterion) is $\|A\|_{2}=\sup _{\|x\|_{2}=1}\|A x\|_{2}=\sqrt{\sigma_{1}^{2}}$, where $\|x\|_{2}$ is the Euclidean norm of a vector $x$. The spectral norm is bounded above by the Frobenius norm since $\|A\|_{2}^{2}=\left|\sigma_{1}\right|^{2} \leq \sum_{i=1}^{n} \sum_{j=1}^{d}\left|A_{i j}\right|^{2}=\|A\|_{F}^{2}=\sum_{i=1}^{d} \sigma_{i}^{2}$.

Let $f$ and $g$ be real valued functions defined on some unbounded subset of real positive numbers $n$. We say that $g(n)=O(f(n))$ if $|g(n)| \leq k|f(n)|$ for some constant $k$ for all $n \geq n_{0}$. This means that $g(n)$ is at most a constant multiple of $f(n)$ for sufficiently large values of $n$. We say that $g(n)=\Omega(f(n))$ if $g(n) \geq k f(n)$ for all $n \geq n_{0}$. This means that $g(n)$ is at least $k f(n)$ for some constant $k$. We say that $g(n)=\Theta(f(n))$ if $k_{1} f(n) \leq g(n) \leq k_{2} f(n)$ for all $n \geq n_{0}$. This means that $g(n)$ is at least $k_{1} f(n)$ and at most $k_{2} f(n)$.

\subsection{Approximate Matrix Multiplication}

Suppose we are given two matrices, $A \in \mathbb{R}^{n \times d}$ and $B \in \mathbb{R}^{n \times p}$ and are interested in the $d \times p$ matrix $C=A^{T} B$. The textbook approach is to compute each element of $C$ by summing over dot products:

$$
C_{i j}=\left[A^{T} B\right]_{i j}=\sum_{k=1}^{n} A_{i k}^{T} B_{k j} .
$$

Equivalently, each element is the inner product of two vectors $A_{(i)}$ and $B^{(j)}$. Computing the entire $C$ entails three loops through $i \in[1, d], j \in[1, p]$, and $k \in[1, n]$. An algorithmically more efficient approach is to form $C$ from outer products:

$$
C=\underbrace{A^{T} B}_{d \times p}=\sum_{i=1}^{n} \underbrace{A_{(i)}^{T} B_{(i)}}_{(d \times 1) \times(1 \times p)},
$$

making $C$ a sum of $n$ matrices each of rank-1. Viewing $C$ as a sum of $n$ terms suggests to approximate it by summing $m<n$ terms only. But which $m$ amongst the $\frac{n !}{m !(n-m) !}$ possible terms to sum? Consider the following Approximate Matrix Multiplication algorithm (AMM). Let $p_{j}$ be the probability that row $j$ will be sampled. 
Algorithm AMM:

Input: $A \in \mathbb{R}^{n \times d}, B \in \mathbb{R}^{n \times p}, m>0, p=\left(p_{1}, \ldots, p_{n}\right)$.

1 for $s=1: m$ do

$2 \quad$ sample $k_{s} \in[1, \ldots n]$ with probability $p_{k_{s}}$ independently with replacement;

$3 \quad$ set $\widetilde{A}_{(s)}=\frac{1}{\sqrt{m p_{k_{s}}}} A_{\left(k_{s}\right)}$ and $\widetilde{B}_{(s)}=\frac{1}{\sqrt{m p_{k_{s}}}} B_{\left(k_{s}\right)}$

Output: $\widetilde{C}=\widetilde{A}^{T} \widetilde{B}$.

The algorithm essentially produces

$$
\widetilde{C}=(\Pi A)^{T} \Pi B=\frac{1}{m} \sum_{s=1}^{m} \frac{1}{p_{k_{s}}} A_{\left(k_{s}\right)}^{T} B_{\left(k_{s}\right)}
$$

where $k_{s}$ denotes the index for the non-zero entry in the $s$ row of the matrix

$$
\Pi=\frac{1}{\sqrt{m}}\left(\begin{array}{ccccc}
0 & \frac{1}{\sqrt{p_{k_{1}}}} & 0 & \ldots & 0 \\
\ldots & \cdots & \ldots & \ldots & \ldots \\
0 & 0 & \cdots & \frac{1}{\sqrt{p_{k_{m}}}} & 0
\end{array}\right)
$$

The $\Pi$ matrix only has only one non-zero element per row, and the $(i, j)$-th entry $\Pi_{i j}=\frac{1}{\sqrt{m p_{j}}}$ with probability $p_{j}$. In the case of uniform sampling with $p_{k}=\frac{1}{n}$ for all $i$, $\Pi$ reduces to a sampling matrix scaled by $\frac{\sqrt{n}}{\sqrt{m}}$.

While $\widetilde{C}$ defined by (2) is recognized in econometrics as the estimator of Horvitz and Thompson (1952) which uses inverse probability weighting to account for different proportions of observations in stratified sampling, $\widetilde{C}$ is a sketch of $C$ produced by the Monte Carlo algorithm AMM in the theoretical computer science literature $4^{4}$ The Monte-Carlo aspect is easily understood if we take $A$ and $B$ to be $n \times 1$ vectors. Then $A^{T} B=\sum_{i=1}^{n} A_{(i)}^{T} B_{(i)}=\sum_{i=1}^{n} f(i) \approx \int_{0}^{n} f(x) d x=f(a) n$ where the last step follows from mean-value theorem for $0<a<n$. Approximating $f(a)$ by $\frac{1}{m} \sum_{s=1}^{m} f\left(k_{s}\right)$ gives $\frac{n}{m} \sum_{s=1}^{m} f\left(k_{s}\right)$ as the Monte Carlo estimate of $\int_{0}^{n} f(x) d x$.

Two properties of $\widetilde{C}$ produced by AMM are noteworthy. Under independent sampling,

$$
\mathbb{E}\left[\frac{1}{m} \sum_{s=1}^{m} \frac{A_{i k_{s}} B_{k_{s}} j}{p_{k_{s}}}\right]=\frac{1}{m} \sum_{s=1}^{m} \sum_{k=1}^{n} p_{k} \frac{A_{i k} B_{k j}}{p_{k}}=\left[A^{T} B\right]_{i j} .
$$

Hence regardless of the sampling distribution, $\widetilde{C}$ is unbiased. The variance of $\widetilde{C}$ defined in terms of the Frobenius norm is

$$
\mathbb{E}\left[\|\widetilde{C}-C\|_{F}^{2}\right]=\frac{1}{m} \sum_{k=1}^{n} \frac{1}{p_{k}}\left\|A_{(k)}^{T}\right\|_{2}^{2}\left\|B_{(k)}\right\|_{2}^{2}-\frac{1}{m}\|C\|_{F}^{2}
$$

\footnotetext{
${ }^{4}$ In Mitzenmacher and Upfal (2006), a Monte Carlo algorithm is a randomized algorithm that may fail or return an incorrect answer but whose time complexity is deterministic and does not depend on the particular sampling. This contrasts with a Las Vegas algorithm which always returns the correct answer but whose time complexity is random. See also Eriksson-Bique et al. (2011).
} 
which depends on the sampling distribution $p$. Drineas, Kannan and Mahoney (2006, Theorem 1) shows that minimizing $\sum_{k=1}^{n} \frac{1}{p_{k}}\left\|A_{(k)}^{T}\right\|_{2}^{2}\left\|B_{(k)}\right\|_{2}^{2}$ with respect to $p$ subject to the constraint $\sum_{k=1}^{n} p_{k}=1$ gives 5

$$
p_{k}=\frac{\left\|A_{(k)}\right\|_{2}\left\|B_{(k)}\right\|_{2}}{\sum_{s=1}^{n}\left\|A_{(s)}\right\|_{2}\left\|B_{(s)}\right\|_{2}} .
$$

This optimal $p$ yields a variance of

$$
\mathbb{E}\left[\|\widetilde{C}-C\|_{F}^{2}\right] \leq \frac{1}{m}\left[\sum_{k=1}^{n}\left\|A_{(k)}\right\|_{2}\left\|B_{(k)}\right\|_{2}\right]^{2} \leq \frac{1}{m}\|A\|_{F}^{2}\|B\|_{F}^{2} .
$$

It follows from Markov's inequality that for given error of size $\varepsilon$ and failure probability $\delta>0$,

$$
P\left(\|\widetilde{C}-C\|_{F}^{2}>\varepsilon^{2}\|A\|_{F}^{2}\|B\|_{F}^{2}\right)<\frac{\mathbb{E}\left[\|\widetilde{C}-C\|_{F}^{2}\right]}{\varepsilon^{2}\|A\|_{F}^{2}\|B\|_{F}^{2}}<\frac{1}{m \varepsilon^{2}},
$$

implying that to have an approximation error no larger than $\varepsilon$ with probability $1-\delta$, the number of rows used in the approximation must satisfy $m=\Omega\left(\frac{1}{\delta \varepsilon^{2}}\right)$.

The approximate matrix multiplication result $\left\|A^{T} B-\widetilde{A}^{T} \widetilde{B}\right\|_{F} \leq \varepsilon\|A\|_{F}\|B\|_{F}$ is the building block of many of the theoretical results to follow. The result also holds under the spectral norm since it is upper bounded by the Frobenius norm. Since $A_{(i)}^{T} B_{(i)}$ is a rank one matrix, $\left\|A_{(i)}^{T} B_{(i)}\right\|_{2}=$ $\left\|A_{(i)}^{T}\right\|_{2}\left\|B_{(i)}\right\|_{2}$. Many of the results to follow are in spectral norm because it is simpler to work with a product of two Euclidean vector norms. Furthermore, putting $A=B$, we have

$$
\left.P\left(\|(\Pi A)^{T}(\Pi A)-A^{T} A\right)\left\|_{2} \geq \epsilon\right\| A \|_{2}^{2}\right)<\delta .
$$

One may think of the goal of AMM as preserving the second moment properties of $A$. The challenge in practice is to understand the conditions that validate the approximation. For example, even though uniform sampling is the simplest of sampling schemes, it cannot be used blindly. Intuitively, uniform sampling treats all data points equally, and when information in the rows are not uniformly dispersed, the influential rows will likely be omitted. From the above derivations, we see that $\operatorname{var}(\widetilde{C})=O\left(\frac{n}{m}\right)$ when $p_{k}=\frac{1}{n}$, which can be prohibitively large. The Mincer equation in the Introduction illustrates the pitfall with uniform sampling when $m$ is too small, but that the problem can by and large be alleviated when $m>2000$. Hence, care must be taken in using the algorithmic sampling schemes. We will provide some guides below.

\footnotetext{
${ }^{5}$ The first order condition satifies $0=-\frac{1}{p_{k}^{2}}\left\|A_{(k)}^{T}\right\|_{2}^{2}\left\|B_{(k)}\right\|_{2}^{2}+\lambda$. Solving for $\sqrt{\lambda}$ and imposing the constraint gives the result stated. Eriksson-Bique et al. (2011) derives probabilities that minimize expected variance for given distribution of the matrix elements.
} 


\subsection{Subspace Embedding}

To study the properties of the least squares estimates using sketched data, we first need to make clear what features of $A$ need to be preserved in $\widetilde{A}$. Formally, the requirement is that $\Pi$ has a 'subspace embedding' property. An embedding is a linear transformation of the data that has the Johnson-Lindenstrauss (JL) property, and a subspace embedding is a matrix generalization of an embedding. Hence it is useful to start with the celebrated JL Lemma.

The JL Lemma, due to Johnson and Lindenstauss (1994), is usually written for linear maps that reduce the number of columns from an $n \times d$ matrix (i.e., $d$ ) to $k$. Given that our interest is ultimately in reducing the number of rows from $n$ to $m$ while keeping $d$ fixed, we state the JL Lemma as follows:

Lemma 1 (JL Lemma) Let $0<\epsilon<1$ and $\left\{a_{1}, \ldots, a_{d}\right\}$ be a set of $d$ points in $\mathbb{R}^{n}$ with $n>d$. Let $m \geq 8 \log d / \epsilon^{2}$. There exists a linear map $\Pi: \mathbb{R}^{n} \rightarrow \mathbb{R}^{m}$ such that $\forall a_{i}, a_{j}$

$$
(1-\epsilon)\left\|a_{i}-a_{j}\right\|_{2}^{2} \leq\left\|\Pi a_{i}-\Pi a_{j}\right\|_{2}^{2} \leq(1+\epsilon)\left\|a_{i}-a_{j}\right\|_{2}^{2}
$$

In words, the Lemma states that every set of $d$ points in Euclidean space of dimension $n$ can be represented by a Euclidean space of dimension $m=\Omega\left(\log d / \epsilon^{2}\right)$ with all pairwise distances preserved up to a $1 \pm \epsilon$ factor. Notice that $m$ is logarithmic in $d$ and does not depend on $n$. A sketch of the proof is given in the Appendix.

The JL Lemma establishes that $d$ vectors in $\mathbb{R}^{n}$ can be embedded into $m=\Omega\left(\log d / \epsilon^{2}\right)$ dimensions. But there are situations when we need to preserve the information in the $d$ columns jointly. This leads to the notion of 'subspace embedding' which requires that the norm of vectors in the column space of $A$ be approximately preserved by $\Pi$ with high probability.

Definition 1 (Subspace-Embedding) Let $A$ be an $n \times d$ matrix. An $L_{2}$ subspace embedding for the column space of $A$ is an $m(\epsilon, \delta, d) \times n$ matrix $\Pi$ such that $\forall x \in \mathbb{R}^{d}$,

$$
(1-\epsilon)\|A x\|_{2}^{2} \leq\|\Pi A x\|_{2}^{2} \leq(1+\epsilon)\|A x\|_{2}^{2}
$$

Subspace embedding is an important concept and it is useful to understand it from different perspectives. Given that $\|A x\|_{2}^{2}=x^{T} A^{T} A x$, preserving the column space of $A$ means preserving the information in $A^{T} A$. The result can analogously be written as

$$
\|\Pi A x\|_{2}^{2} \in\left[(1-\epsilon)\|A x\|_{2}^{2},(1+\epsilon)\|A x\|_{2}^{2}\right] .
$$


Since $A x=U \Sigma V^{T} x=U z$ where $z=\Sigma V^{T} x \in \mathbb{R}^{d}$ and $U$ is orthonormal, a change of basis gives:

$$
\begin{aligned}
\|\Pi U z\|_{2}^{2} & \in\left[(1-\epsilon)\|U z\|_{2}^{2},(1+\epsilon)\|U z\|_{2}^{2}\right] \\
& =\left[(1-\epsilon)\|z\|_{2}^{2},(1+\epsilon)\|z\|_{2}^{2}\right] \\
& \Leftrightarrow\left\|(\Pi U)^{T}(\Pi U)-U^{T} U\right\|_{2} \leq \epsilon \\
& \Leftrightarrow z^{T}\left((\Pi U)^{T}(\Pi U)-I_{d}\right) z \leq \epsilon .
\end{aligned}
$$

The following Lemma defines subspace embedding in terms of singular value distortions.

Lemma 2 Let $U \in \mathbb{R}^{n \times d}$ be a unitary matrix and $\Pi$ be a subspace embedding for the column space of $A$. Let $\sigma_{k}$ is the $k$-th singular value of $A$. Then (3) is equivalent to

$$
\sigma_{k}^{2}(\Pi U) \in[1-\epsilon, 1+\epsilon] \quad \forall k \in[1, d]
$$

To understand Lemma 2, consider the Rayleigh quotient form of $\Pi U{ }^{6}$

$$
\omega_{k}\left((\Pi U)^{T}(\Pi U)\right)=\frac{v_{k}^{T}(\Pi U)^{T}(\Pi U) v_{k}}{v_{k}^{T} v_{k}}
$$

for some vector $v_{k} \neq 0$. As $\omega_{k}\left(A^{T} A\right)=\sigma_{k}^{2}(A)$,

$$
\begin{aligned}
\omega_{k}\left((\Pi U)^{T}(\Pi U)\right) & =\frac{v_{k}^{T} v_{k}-v_{k}^{T}\left(I_{d}-(\Pi U)^{T}(\Pi U)\right) v_{k}}{v_{k}^{T} v_{k}} \\
& =1-\omega_{k}\left(I_{d}-(\Pi U)^{T}(\Pi U)\right) .
\end{aligned}
$$

This implies that $\left|1-\sigma_{k}^{2}(\Pi U)\right|=\left|\omega_{k}\left(I_{d}-(\Pi U)^{T}(\Pi U)\right)\right|=\sigma_{k}\left(I_{d}-(\Pi U)^{T}(\Pi U)\right)$. It follows that

$$
\begin{aligned}
\left|1-\sigma_{k}^{2}(\Pi U)\right| & =\left|\sigma_{k}\left(U^{T} U-(\Pi U)^{T}(\Pi U)\right)\right| \\
& \leq \sigma_{\max }\left(U^{T} U-(\Pi U)^{T}\left(\Pi^{T} U\right)\right) \\
& =\left\|U^{T} U-(\Pi U)^{T}(\Pi U)\right\|_{2} \leq \epsilon \\
\Leftrightarrow \sigma_{k}^{2}(\Pi U) & \in[1-\epsilon, 1+\epsilon] \quad \forall k \in[1, d] .
\end{aligned}
$$

Hence the condition $\left\|(\Pi U)^{T} \Pi U-I_{d}\right\|_{2} \leq \epsilon$ is equivalent to $\Pi$ generating small singular value distortions. Nelson and Nguyen (2013a) relates this condition to similar results in random matrix theory. ${ }^{7}$

\footnotetext{
${ }^{6}$ For a Hermitian matrix $M$, the Rayleigh quotient is $\frac{c^{T} M c}{c^{T} c}$ for a nonzero vector $c$. By Rayleigh-Ritz Theorem, $\min (\sigma(M)) \leq \frac{c^{T} M c}{c^{T} c} \leq \max (\sigma(M))$ with equalities when $c$ is the eigenvector corresponding to the smallest and largest eigenvalues of $M$, respectively. See, e.g. Hogben (2007, Section 8.2).

${ }^{7}$ Consider a $T \times N$ matrix of random variables with mean zero and unit variance with $c=\lim _{N, T \rightarrow \infty} \frac{N}{T}$. In random matrix theory, the largest and smallest eigenvalues of the sample covariance matrix have been shown to converge to $(1+\sqrt{c})^{2},(1-\sqrt{c})^{2}$, respectively. See, e.g., Yin et al. (1988) and Bai and Yin (1993).
} 
But where to find these embedding matrices? We can look for data dependent or data oblivious ones. We say that $\Pi$ is a data oblivious embedding if it can be designed without knowledge of the input matrix. The idea of oblivious subspace-embedding first appeared in Sarlos (2006) in which it is suggested that $\Pi$ can be drawn from a distribution with the JL properties.

Definition 2 A random matrix $\Pi \in \mathbb{R}^{m \times n}$ drawn from a distribution $F$ forms a JL transform with parameters $\epsilon, \delta, d$ if there exists a function $f$ such that for any $0 \leq \epsilon, \delta \leq 1$ and $m=\Omega\left(\log \left(\frac{d}{\epsilon^{2}} f(\delta)\right)\right)$, $(1-\epsilon)\|x\|_{2}^{2} \leq\|\Pi x\|_{2}^{2} \leq(1+\epsilon)\|x\|_{2}^{2}$ holds with probability at least $1-\delta$ for all d-vector $x \subset \mathbb{R}^{n}$.

A JL transform is often written $\operatorname{JLT}(\epsilon, \delta, d)$ for short. Embedding matrices $\Pi$ that are JLT guarantee good approximation to matrix products in terms of Frobenius norm. This means that for such Пs, an $m$ can be chosen such that for conformable matrices $A, B$ having $n$ rows:

$$
P\left(\left\|(\Pi A)^{T}(\Pi B)-A^{T} B\right\|_{F} \leq \epsilon\|A\|_{F}\|B\|_{F}\right) \geq 1-\delta .
$$

The Frobenius norm bound has many uses. If $A=B$, then $\|\Pi A\|_{F}^{2}=(1 \pm \epsilon)\|A\|_{F}^{2}$ with high probability. The result also holds in the spectral norm, Sarlos (2006, Corollary 11).

\section{Random Sampling, Random Projections, and the Countsketch}

There are two classes of $\Pi s$ with the JL property: random sampling which reduces the row dimension by randomly picking rows of $A$, and random projections which form linear combinations from the rows of $A$. A scheme known as a countsketch that is not a JL transform can also achieve subspace embedding efficiently. We will use a pen and pencil example with $m=3$ and $n=9$ to provide a better understanding of the three types of $\Pi$ s. In this example, $A$ has 9 rows given by $A_{1}, \ldots, A_{9}$.

\subsection{Random Sampling (RS)}

Let $D$ be a diagonal rescaling matrix with $\frac{1}{\sqrt{m p_{i}}}$ in the $i$-th diagonal and $p_{i}$ is the probability that row $i$ is chosen. Under random sampling,

$$
\Pi=D S,
$$

where $S_{j k}=1$ if row $k$ is selected in the $j$-th draw and zero otherwise so that the $j$-th row of the selection matrix $S$ is the $j$ th-row of an $n$ dimensional indentity matrix. Examples of sampling schemes are:

RS1. Uniform sampling without replacement: $\Pi \in \mathbb{R}^{m \times n}, D \in \mathbb{R}^{m \times m}, p_{i}=\frac{1}{n}$ for all $i$. Each row is sampled at most once. 
RS2. Uniform sampling with replacement: $\Pi \in \mathbb{R}^{m \times n}, D \in \mathbb{R}^{m \times m}, p_{i}=\frac{1}{n}$ for all $i$. Each row can be sampled more than once.

RS3. Bernoulli sampling uses an $n \times n$ matrix $\Pi=D S$, where $D=\sqrt{\frac{n}{m}} I_{n}, S$ is initialized to be $0_{n \times n}$ and the $j$-th diagonal entry is updated by

$$
S_{j j}=\left\{\begin{array}{l}
1 \quad \text { with probability } \frac{m}{n} \\
0 \quad \text { with probability } 1-\frac{m}{n}
\end{array}\right.
$$

Each row is sampled at most once, and $m$ is the expected number of sampled rows.

RS4. Leverage score sampling: the sampling probabilities are taken from importance sampling distribution

$$
p_{i}=\frac{\ell_{i}}{\sum_{i=1}^{n} \ell_{i}}=\frac{\ell_{i}}{d}
$$

where for $A$ with $\operatorname{SvD}(A)=U D V^{T}$,

$$
\ell_{i}=\left\|U_{(i)}\right\|_{2}^{2}=\left\|e_{i}^{T} U\right\|_{2}^{2}
$$

is the leverage score for row $i, \sum_{i} \ell_{i}=\|U\|_{F}^{2}=d$, and $e_{i}$ is a standard basis vector.

Notably, the rows of the sketch produced by random sampling are the rows of the original matrix $A$. For example, If rows 9,5,1 are randomly chosen by uniform sampling, RS1 would give

$$
\widetilde{A}=D\left(\begin{array}{ccccccccc}
0 & 0 & 0 & 0 & 0 & 0 & 0 & 0 & 1 \\
0 & 0 & 0 & 0 & 1 & 0 & 0 & 0 & 0 \\
1 & 0 & 0 & 0 & 0 & 0 & 0 & 0 & 0
\end{array}\right) A=\frac{\sqrt{9}}{\sqrt{3}}\left(\begin{array}{c}
A_{9} \\
A_{5} \\
A_{1}
\end{array}\right)
$$

Ipsen and Wentworth 2014, Section 3.3) shows that sampling schemes RS1-RS3 are similar in terms of the condition number and rank deficiency in the matrices that are being subsampled. Unlike these three sampling schemes, leverage score sampling is not data oblivious and warrants further explanation.

As noted above, uniform sampling may not be efficient. More precisely, uniform sampling does not work well when the data have high coherence, where coherence refers to the maximum of the row leverage scores $\ell_{i}$ defined above. Early work suggests to use sampling weights that depend on the Euclidean norm, $p_{i}=\frac{\left\|A_{i}\right\|_{2}^{2}}{\|A\|_{F}^{2}}$. See, e.g., Drineas, Kannan and Mahoney (2006) and Drineas and Mahoney (2005). Subsequent work finds that a better approach is to sample according to the leverage scores which measure the correlation between the left singular vectors of $A$ with the standard basis, and thus indicates whether or not information is spread out. The idea of leverage-sampling, first used in Jolliffe (1972), is to sampling a row more frequently if it has more information.8 Of

\footnotetext{
${ }^{8}$ There are other variations of leverage score sampling. McWilliams et al. (2014) considers subsampling in linear regression models when the observations of the covariances may be corrupted by an additive noise. The influence of observation $i$ is defined by $d_{i}=\frac{e_{i}^{2} \ell_{i}}{\left(1-\ell_{i}\right)^{2}}$, where $e_{i}$ is the OLS residual and $\ell_{i}$ is the leverage score. Unlike leverage scores, $d_{i}$ takes into account the relation between the predictor variables and the $y$.
} 
course, $\ell_{i}$ is simply the $i$-th diagonal element of the hat matrix $A\left(A^{T} A\right)^{-1} A^{T}$, known to contain information about influential observations. In practice, computation of the leverage scores requires an eigen decomposition which is itself expensive. Drineas et al. (2012) and Cohen, Lee, Musco, Musco, Peng and Sidford (2015) suggest fast approximation of leverage scores.

\subsection{Random Projections (RP)}

Some examples of random projections are:

RP1. Gaussian: $\Pi \in \mathbb{R}^{m \times n}$ where $\Pi_{i j}=\frac{1}{\sqrt{m}} N(0,1)$.

RP2. Rademacher random variables: with entries of $\{+1,-1\}$, Sarlos (2006), Achiloptas (2003).

RP3. Randomized Orthogonal Systems: $\Pi=\sqrt{\frac{n}{m}} P H D$ where $D$ is an $n \times n$ is diagonal Rademacher matrix with entries of $\pm 1, P$ is a sparse matrix, and $H$ is an orthonormal matrix.

RP4. Sparse Random Projections (SRP)

$$
\Pi=D S
$$

where $D \in \mathbb{R}^{m \times m}$ is a diagonal matrix of $\sqrt{\frac{s}{m}}$ and $S \in \mathbb{R}^{m \times n}$

$$
S_{i j}= \begin{cases}-1 & \text { with probability } \frac{1}{2 s} \\ 0 & \text { with probability } 1-\frac{1}{s} \\ 1 & \text { with probability } \frac{1}{2 s}\end{cases}
$$

RP1 and RP2 form sub-Gaussian random projections 99 The rows of the sketch produced by random projections are linear combinations of the rows of the original matrix. For example, RP4 with $s=3$ could give

$$
\widetilde{A}=D\left(\begin{array}{rrrrrrrrr}
0 & 0 & 1 & 1 & 0 & -1 & 0 & 0 & 0 \\
1 & 0 & -1 & 0 & -1 & 0 & 0 & 0 & 1 \\
0 & 1 & 0 & 0 & 0 & 0 & 1 & 0 & -1
\end{array}\right) A=\frac{\sqrt{3}}{\sqrt{9}}\left(\begin{array}{c}
A_{3}+A_{4}-A_{6} \\
A_{1}-A_{3}-A_{5}+A_{9} \\
A_{2}+A_{7}-A_{9}
\end{array}\right)
$$

Early work on random projections such as Dasgupta et al. (2010) uses ПIs that are dense, an example being RP1. Subsequent work favors sparser Пs, an example being RP4. Achiloptas (2003) initially considers $s=3$. Li et al. (2006) suggests to increase $s$ to $\sqrt{n}$. Given that uniform sampling is algorithmically inefficient when information is concentrated, the idea of randomized orthogonal systems is to first randomize the data by the matrix $H$ to destroy uniformity, so that sampling in a data oblivious manner using $P$ and rescaling by $D$ remains appropriate. The randomization step is

\footnotetext{
${ }^{9} \mathrm{~A}$ mean-zero vector $s \in \mathbb{R}^{n}$ is sub-Gaussian if for any $u \in \mathbb{R}^{n}$ and for all $\epsilon>0, P\left\{\left|u^{T} s\right| \geq \epsilon\|u\|_{2}\right\} \leq 2 e^{-\epsilon^{2} / K^{2}}$ for some absolute constant $K>0$.
} 
sometimes referred to as 'preconditioning'. Common choices of $H$ are the Hadamard matrix as in the SRHT of Ailon and Chazelle $(2009){ }^{10}$ and the discrete Fourier transform as in FJLT of Woolfe et al. (2008).

\subsection{Countsketch}

While sparse $\Pi$ s reduce computation cost, Kane and Nelson (2014, Theorem 2.3) shows that each column of $\Pi$ must have $\Theta(d / \epsilon)$ non-zero entries to create an $L_{2}$ subspace embedding. This would seem to suggest that $\Pi$ cannot be too sparse. However, Clarkson and Woodruff (2013) argues that if the non-zero entries of $\Pi$ are carefully chosen, $\Pi$ need not be a JLT and a very sparse subspace embedding is actually possible. Their insight is that $\Pi$ need not preserve the norms of an arbitrary subset of vectors in $\mathbb{R}^{n}$, but only those that sit in the $d$-dimensional subspace of $\mathbb{R}^{n}$. The sparse embedding matrix considered in Clarkson and Woodruff (2013) is the countsketch 11

A countsketch of sketching dimension $m$ is a random linear map $\Pi=P D: \mathbb{R}^{n} \rightarrow \mathbb{R}^{m}$ where $D$ is an $n \times n$ random diagonal matrix with entries chosen independently to be +1 or -1 with equal probability. Furthermore, $P \in\{0,1\}$ is an $m \times n$ binary matrix such that $P_{h(i), i}=1$ and $P_{j, i}=0$ for all $j \neq h(i)$, and $h:[n] \rightarrow[m]$ is a random map such that for each $i \in[n], h(i)=m^{\prime}$ for $m^{\prime} \in[m]$ with probability $\frac{1}{m}$. As an example, a countsketch might be

$$
\widetilde{A}=\left(\begin{array}{rrrrrrrrr}
0 & 0 & 1 & 0 & 1 & -1 & 0 & 0 & 1 \\
-1 & 0 & 0 & -1 & 0 & 0 & 0 & -1 & 0 \\
0 & -1 & 0 & 0 & 0 & 0 & 1 & 0 & 0
\end{array}\right) A=\left(\begin{array}{c}
A_{3}+A_{5}-A_{6}+A_{9} \\
-A_{1}-A_{4}-A_{8} \\
-A_{2}+A_{7}
\end{array}\right)
$$

Like random projections, the rows of a countsketch are also a linear combinations of the rows of $A$.

Though the countsketch is not a JLT, Nelson and Nguyen (2013b) and Meng and Mahoney (2013) show that the following spectral norm bound holds for the countsketch with appropriate choice of $m$ :

$$
P\left(\left\|(\Pi U)^{T}(\Pi U)-I_{d}\right\|_{2}>3 \epsilon\right) \leq \delta
$$

which implies that countsketch provides a $1+\varepsilon$ subspace embedding for the column space of $A$ in spite of not being a JLT, see Woodruff (2014, Theorem 2.6).

The main appeal of the countsketch is that the run time needed to compute $\Pi A$ can be reduced to $O(\operatorname{NNZ}(A))$, where $\operatorname{Nnz}(\mathrm{A})$ denotes the number of non-zero entries of $A$. The efficiency gain is due to extreme sparsity of a countsketch $\Pi$ which only has one non-zero element per column. Still,

\footnotetext{
${ }^{10}$ The Hadamard matrix is defined recursively by $H_{n}=\left(\begin{array}{cc}H_{n / 2} & H_{n / 2} \\ H_{n / 2} & -H_{n / 2}\end{array}\right), \quad H_{2}=\left(\begin{array}{cc}1 & 1 \\ 1 & -1\end{array}\right)$. A constraint is that $n$ must be in powers of two.

${ }^{11}$ The definition is taken from Dahiya et al. (2018). Given input $j$, a count-sketch matrix can also be characterized by a hash function $h(j)$ such that $\forall j, j^{\prime}, j \neq j^{\prime} \rightarrow h(j) \neq h\left(j^{\prime}\right)$. Then $\Pi_{h(j), j}= \pm 1$ with equal probability $1 / 2$.
} 
the $\Pi$ matrix can be costly to store when $n$ is large. Fortunately, it is possible to compute the sketch without constructing $\Pi$.

The streaming version of the countsketch is a variant of the frequent-items algorithm where we recall that having to compute summaries such as the most frequent item in the data that stream by was instrumental to the development of sketching algorithms. The streaming algorithm proceeds by initializing $\widetilde{A}$ to an $m \times n$ matrix of zeros. Each row $A_{(i)}$ of $A$ is updated as

$$
\widetilde{A}_{h(i)}=\widetilde{A}_{h(i)}+g(i) A_{(i)}
$$

where $h(i)$ sampled uniformly at random from $[1,2, \ldots m]$ and $g_{i}$ sampled from $\{+1,-1\}$ are independent. Computation can be done one row at a time 12 The Appendix provides the streaming implementation of the example above.

\subsection{Properties of the $\Pi s$}

To assess the actual performance of the different Пs, we conduct a small Monte Carlo experiment with 1000 replications. For each replication $b$, we simulate an $n \times d$ matrix $A$ and construct the seven JL embeddings considered above. For each embedding, we count the number of times that $\left\|\mid \Pi\left(a_{i}-a_{j}\right)\right\|_{2}^{2}$ is within $(1 \pm \epsilon)$ of $\left\|a_{i}-a_{j}\right\|_{2}^{2}$ for all $d(d+1) / 2$ pairs of distinct $(i, j)$. The success rate for the replication is the total count divided by $d\left((d+1) / 2\right.$. We also record $\left\|\frac{\sigma(\Pi A)}{\sigma(A)}-1\right\|_{2}$ where $\sigma(\Pi A)$ is a vector of $d$ singular values of $\Pi A$. According to theory, the pairwise distortion of the vectors should be small if $m \geq C \log d / \epsilon^{2}$. We set $(n, d)=(20,000)$ and $\epsilon=0.1$. Four values of $C=\{1,2,3,4,5,6,8,16\}$ are considered. We draw $A$ from the (i) normal distribution, and (ii) the exponential distribution. In MATLAB, these are generated as $\mathrm{X}=\mathrm{RANDN}(\mathrm{N}, \mathrm{D})$ and $\mathrm{X}=\operatorname{EXPRnd}(\mathrm{D},[\mathrm{N} \mathrm{D}])$. Results for the Pearson distribution using $\mathrm{X}=\operatorname{PEARSRND}(0,1,1,5, \mathrm{~N}, \mathrm{D})$ are similar and not reported.

Table 1 reports the results averaged over 1000 simulations. With probability around 0.975 , the pairwise distance between columns with 1000 rows is close to the pairwise distance between columns with 20000 rows. The average singular value distortion also levels off with about 1000 rows of data. Hence, information in $n$ rows can be well summarized by a smaller matrix with $m$ rows. However, note that more rows are generally needed for uniform sampling, while fewer rows are needed for leverage score sampling, to have the same error as the remaining methods. The takeaway from Table 1 is that the performance of the different $\Pi$ s are quite similar, making computation cost and analytical tractability two important factor in deciding which ones to use.

\footnotetext{
${ }^{12}$ See Ghashami et al. (2016). Similar schemes have been proposed in Charika et al. (2002); Conmode and Muthukrishnan (2005).
} 
Choosing a $\Pi$ is akin to choosing a kernel function in nonparametric regression and many will work well, but there are analytical differences. Any $\Pi^{T} \Pi$ can be written as $I_{n}+R_{11}+R_{12}$ where $R_{11}$ is a generic diagonal and $R_{12}$ is a generic $n \times n$ matrix with zeros in each diagonal entry. Two features will be particularly useful.

$$
\begin{aligned}
\Pi^{T} \Pi & =I_{n}+R_{11} \\
\Pi \Pi^{T} & =\frac{n}{m} I_{m}
\end{aligned}
$$

Property $7 \mathrm{a}$ imposes that $\Pi^{T} \Pi$ is a diagonal matrix, allowing $R_{11} \neq 0$ but restricting $R_{12}=0$. Each $\Pi \Pi^{T}$ can also be written as $\Pi \Pi^{T}=\frac{n}{m} I_{m}+R_{21}+R_{22}$ where $R_{21}$ is a generic diagonal and $R_{22}$ is a generic $m \times m$ matrix with zeros in each diagonal entry. Property $7 \mathrm{~b}$ requires that $\Pi^{T}$ is proportional to an identity matrix, and hence that $R_{21}=R_{22}=0_{m \times m}$.

For the Пis previously considered, we summarize their properties as follows:

\begin{tabular}{l|ll}
\hline & $(7 \mathrm{a})$ & $\overline{7 b})$ \\
\hline RS1 (Uniform,w/o) & yes & yes \\
RS2 (Uniform,w) & yes & no \\
RS3 (Bernoulli) & yes & no \\
RS4 (Leverage) & yes & no \\
RP1 (Gaussian) & no & no \\
RP2 (Rademacher) & no & no \\
RP3 (SRHT) & yes & yes \\
RP4 (SRP) & no & no \\
CS (Countsketch) & no & no \\
\hline
\end{tabular}

Property (7a) holds for all three random sampling methods but of all the random projection methods considered, the property only holds for SRHT. This is because SRHT effectively performs uniform sampling of the randomized data. For property $(7 \mathrm{~b})$, it is easy to see that $R_{21}=0$ and $R_{22}=$ $0_{m \times m}$ when uniform sampling is done without replacement and $\Pi \Pi^{T}=\frac{n}{m} I_{m}$. By implication, the condition also holds for SRHT if sampling is done without replacement since $H$ and $D$ are orthonormal matrices. But uniform sampling is computationally much cheaper than SRHT and has the distinct advantage over the SRHT that the rows of the sketch are those of the original matrix and hence interpretable. For this reason, we will subsequently focus on uniform sampling and use its special structure to obtain precise statistical results. Though neither condition holds for the countsketch, the computation advantage due to its extreme sparse structure makes it worthy of further investigation. 


\section{Algorithmic Results for the Linear Regression Model}

The linear regression model with $K$ regressors is $y=X \beta+e$. The least squares estimator minimizes $\|y-X b\|_{2}$ with respect to $b$ and is defined by

$$
\hat{\beta}=\left(X^{T} X\right)^{-1} X^{T} y=V \Sigma^{-1} U^{T} y
$$

We are familiar with the statistical properties of $\hat{\beta}$ under assumptions about $e$ and $X$. But even without specifying a probabilistic structure, $\|y-X b\|_{2}$ with $n>K$ is an over-determined system of equations and can be solved by algebraically. The SVD solution gives $\beta^{*}=X^{-} y$ where $\operatorname{SvD}(X)=$ $U \Sigma V^{T}$, the pseudoinverse is $X^{-}=V \Sigma^{-1} U^{T}$. The 'Choleski' solution starts with the normal equations $X^{T} X \beta=X^{T} y$ and factorizes $X^{T} X$. The algebraic properties of these solutions are well studied in the matrix computations literature when all data are used.

Given an embedding matrix $\Pi$ and sketched data $(\Pi y, \Pi X)$, minimizing $\|\Pi(y-X b)\|_{2}^{2}$ with respect to $b$ gives the sketched estimator

$$
\widetilde{\beta}=\left((\Pi X)^{T} \Pi X\right)^{-1}(\Pi X)^{T} \Pi y .
$$

Let $\widehat{\mathrm{SSR}}=\|y-X \hat{\beta}\|_{2}^{2}$ be the full sample sum of squared residuals. For an embedding matrix $\Pi \in \mathbb{R}^{m \times n}$, let $\widetilde{\mathrm{SSR}}=\|\widetilde{y}-\widetilde{X} \widetilde{\beta}\|_{2}^{2}$ be the sum of squared residuals from using the sketched data. Assume that the following two conditions hold with probability $1-\delta$ for $0<\epsilon<1$ :

$$
\begin{aligned}
&\left|1-\sigma_{k}^{2}(\Pi U)\right| \leq \frac{1}{\sqrt{2}} \forall k=1, \ldots, K ; \\
&\left\|(\Pi U)^{T} \Pi(y-X \hat{\beta})\right\|_{2}^{2} \leq \epsilon \widehat{\mathrm{SSR}}^{2} / 2 .
\end{aligned}
$$

Condition $8 \mathrm{a}$ is is equivalent to $\left\|(\Pi U)^{T}(\Pi U)-U^{T} U\right\|_{2} \leq \frac{1}{\sqrt{2}}$ as discussed above. Since $\sigma_{i}(U)=1$ for all $k \in[1, K]$, the condition requires the smallest singular value, $\sigma_{K}(\Pi U)$, to be positive so that $\Pi X$ has the same rank as $X$. A property of the least squares estimator is for the least squares residuals to be orthogonal to $X$, ie. $U^{T}(y-X \hat{\beta})=0$. Condition $8 \mathrm{~b}$ requires near orthogonality when both quantities are multiplied by $\Pi$. The two algorithmic features of sketched least squares estimation are summarized below.

Lemma 3 Let the sketched data be $(\Pi y, \Pi X)=(\widetilde{y}, \widetilde{X})$ where $\Pi \in \mathbb{R}^{m \times n}$ is a subspace embedding matrix. Let $\sigma_{\min }(X)$ be the smallest singular value of $X$. Suppose that conditions 8a) and 8b hold. Then with probability at least $(1-\delta)$ and for suitable choice of $m$,

(i). $\widetilde{\mathrm{SSR}} \leq(1+\epsilon) \widehat{\mathrm{SSR}}$;

(ii). $\|\widetilde{\beta}-\hat{\beta}\|_{2} \leq \epsilon \cdot \widehat{\mathrm{SSR}} / \sigma_{\min }$. 
Sarlos (2006) provides the proof for random projections, while Drineas, Mahoney and Muthukrishnan (2006) analyzes the case of leverage score sampling. The desired $m$ depends on the result and the sampling scheme.

Part (i) is based on subspace embedding arguments. By optimality of $\widetilde{\beta}$ and JL Lemma,

$$
\begin{aligned}
\widetilde{\mathrm{SSR}} & =\|\left.\Pi(y-X \widetilde{\beta})\right|_{2} \\
& \leq\|\Pi(y-X \hat{\beta})\|_{2} \quad \text { by optimality of } \widetilde{\beta} \\
& \leq(1+\epsilon)\|y-X \hat{\beta}\|_{2} \quad \text { by subspace embedding } \\
& =(1+\epsilon) \widehat{\mathrm{SSR}} .
\end{aligned}
$$

Part (ii) shows that the sketching error is data dependent. Consider a reparameterization of $X \hat{\beta}=U \Sigma V^{T} \hat{\beta}=U \hat{\theta}$ and $X \widetilde{\beta}=U \Sigma V^{T} \widetilde{\beta}=U \widetilde{\theta}$. As shown in the Appendix, $\|\widetilde{\theta}-\hat{\theta}\|_{2} \leq \sqrt{\epsilon} \widehat{\operatorname{SSR}}$. Taking norms on both sides of $X(\widetilde{\beta}-\hat{\beta})=U(\widetilde{\theta}-\hat{\theta})$ and since $U$ is orthonormal,

$$
\|\widetilde{\beta}-\hat{\beta}\|_{2} \leq \frac{\|U(\widetilde{\theta}-\hat{\theta})\|_{2}}{\sigma_{\min }} .
$$

Notably, difference between $\hat{\beta}$ and $\widetilde{\beta}$ depends on the minimum singular value of $X$. Recall that for consistent estimation, we also require that the minimum eigenvalue to diverge.

The non-asymptotic worse case error bounds in Lemma 3 are valid for any subspace embedding matrix $\Pi$, though more precise statements are available for certain $\Pi$ s. For leverage score sampling, see Drineas, Mahoney and Muthukrishnan (2006), for uniform sampling and SRHT, see Drineas et al. (2011); and for the countsketch, Woodruff (2014, Theorem 2.16), Meng and Mahoney (2013, Theorem 1), Nelson and Nguyen (2013a). These algorithmic results are derived without reference to the probabilistic structure of the data. Hence the results do not convey information such as bias and sampling uncertainty. An interesting question is whether optimality from an algorithmic perspective implies optimality from a statistical perspective. Using Taylor series expansion, Ma et al. (2014) shows that leverage-based sampling does not dominate uniform sampling in terms of bias and variance, while Raskutti and Mahoney (2016) finds that prediction efficiency requires $m$ to be quite large. Pilanci and Wainwright (2015) shows that the solutions from sketched least squares regressions have larger variance than the oracle solution that uses the full sample. Pilanci and Wainwright (2015) provides a result that relates $m$ to the rank of the matrix. Wang, Gittens and Mahoney (2018) studies four sketching methods in the context of ridge regressions that nests least squares as a special case. It is reported that sketching schemes with near optimal algorithmic properties may have features that not statistically optimal. Chi and Ipsen (2018) decomposes the variance of $\widetilde{\beta}$ into a model induced component and an algorithm induced component. 


\section{$5 \quad$ Statistical Properties of $\widetilde{\beta}$}

We consider the linear regression model with $K$ regressors:

$$
y=X^{T} \beta+e, \quad e_{i} \sim\left(0, \Omega_{e}\right)
$$

where $y$ is the dependent variable, $X$ is the $n \times K$ matrix of regressors, $\beta$ is the $K \times 1$ vector of regression coefficients whose true value is $\beta_{0}$. It should be noted that $K$ is the number of predictors which is generally larger than $d$, which is the number of covariates available since the predictors may include transformation of the $d$ covariates. In the Mincer example, we have data for EDU, EXP collected into $A$ with $d=2$ columns. From these two covariates, $K=4$ regressors are constructed for regression (1a), while $K=14$ regressors are constructed for regression (1b).

The full sample estimator using data $(y, X)$ is $\hat{\beta}=\left(X^{T} X\right)^{-1} X^{T} y$. For a given $\Pi$, the estimator using sketched data $(\widetilde{y}, \widetilde{X})=(\Pi y, \Pi X)$ is

$$
\widetilde{\beta}=\left(\widetilde{X}^{T} \widetilde{X}\right)^{-1} \widetilde{X}^{T} \widetilde{y}
$$

\section{Assumption OLS:}

(i) the regressors $X$ are non-random, has SVD $X=U \Sigma V^{T}$, and $X^{T} X$ is non-singular;

(ii) $\mathbb{E}\left[e_{i}\right]=0$ and $\mathbb{E}\left[e e^{T}\right]=\Omega_{e}$ is a diagonal positive definite matrix.

\section{Assumption PI:}

(i) $\Pi$ is independent of $e$;

(ii) for given singular value distortion parameter $\varepsilon_{\sigma} \in(0,1)$, there exists failure parameter $\delta_{\sigma} \in$ $(0,1)$ such that $P\left(\left|1-\sigma_{k}^{2}(\Pi U)\right| \leq \varepsilon_{\sigma}\right.$ for all $\left.k=1, \ldots K\right) \geq 1-\delta_{\sigma}$.

(iii) $\Pi^{T} \Pi$ is an $n \times n$ diagonal matrix and $\Pi \Pi^{T}=\frac{n}{m} I_{m}$.

Assumption OLS is standard in regression analyses. The errors are allowed to be possibly heteroskedastic but not cross-correlated. Under Assumption OLS, $\hat{\beta}$ is unbiased, i.e. $\mathbb{E}[\hat{\beta}]=\beta_{0}$ with a sandwich variance

$$
\mathbb{V}(\hat{\beta})=\left(X^{T} X\right)^{-1}\left(X^{T} \Omega_{e} X\right)\left(X^{T} X\right)^{-1}
$$

Assumption PI.(i) is needed for $\widetilde{\beta}$ to be unbiased. Assumption PI.(ii) restricts attention to $\Pi$ matrices that have subspace embedding property. As previously noted, the condition is equivalent 
to $\left\|I_{d}-(\Pi U)^{T}(\Pi U)\right\|_{2} \leq \varepsilon_{\sigma}$ holding with probability $1-\delta_{\sigma}$. We use PI2 to refer Assumptions PI (i) and (ii) holding jointly. Results under PI2 are not specific to any $\Pi$.

Assumption PI.(iii) simplifies the expression for $\mathbb{V}(\widetilde{\beta} \mid \Pi)$, the variance of $\widetilde{\beta}$ conditional on $\Pi$, and we will use PI3 to denote Assumptions PI (i)-(iii) holding jointly. PI3 effectively narrows the analysis to uniform sampling and SRHT without replacement. We will focus on uniform sampling without replacement for a number of reasons. It is simple to implement, and unlike the SRHT, the rows have meaningful interpretation. In a regression context, uniform sampling has an added advantage that there is no need to reconstruct $(\widetilde{y}, \widetilde{X})$ each time we add or drop a variable in the $X$ matrix. In contrast, most other $\Pi$ s require $(\widetilde{y}, \widetilde{X})$ to be reconstructed. This can be cumbersome when variable selection is part of the empirical exercise. Uniform sampling without replacement is an exception since the columns are unaffected once the rows are randomly chosen.

For regressions, we need to know not only the error in approximating $X^{T} X$, but also the error in approximating $\left(X^{T} X\right)^{-1}$. This is made precise in the next Lemma.

Lemma 4 Suppose that PI2 is satisfied. For given non-random matrix $X \in \mathbb{R}^{n \times K}$ of full rank with $\operatorname{SvD}(X)=U \Sigma V^{T}$, consider any non-zero $K \times 1$ vector $c$. It holds with probability at least $1-\delta_{\sigma}$ that

$$
\left|\frac{c^{T}\left[\left(X^{T} X\right)^{-1}-\left(\widetilde{X}^{T} \widetilde{X}\right)^{-1}\right] c}{c^{T}\left(X^{T} X\right)^{-1} c}\right| \leq \frac{\varepsilon_{\sigma}}{1-\varepsilon_{\sigma}} .
$$

The Lemma follows from the fact that

$$
\begin{aligned}
\left(X^{T} X\right)^{-1} & =V \Sigma^{-2} V^{T} \equiv P P^{T} \\
\left((\Pi X)^{T}(\Pi X)\right)^{-1} & =P Q P^{T}
\end{aligned}
$$

where $P=V \Sigma^{-1}$ and $Q^{-1}=(\Pi U)^{T}(\Pi U)$. By the property of Rayleigh quotient, the smallest eigenvalue of $\left(U^{T} \Pi^{T} \Pi U\right)$ is bounded below by $\left(1-\varepsilon_{\sigma}\right)$. Hence

$$
\left|\frac{c^{T}\left(P Q P^{T}-P P^{T}\right) c}{c^{T} P P^{T} c}\right|=\left|\frac{c^{T} P\left(I_{d}-Q^{-1}\right) Q P^{T} c}{c^{T} P P^{T} c}\right| \leq\|Q\|_{2}\left\|I_{d}-Q^{-1}\right\|_{2} \leq \frac{\varepsilon_{\sigma}}{\left(1-\varepsilon_{\sigma}\right)} .
$$

The approximation error $\left(X^{T} X\right)^{-1}$ is thus larger than that for $X^{T} X$, which equals $\varepsilon_{\sigma}$.

Under Assumptions OLS and PI3, $\widetilde{\beta}$ is unbiased and has sandwich variance

$$
\mathbb{V}(\widetilde{\beta} \mid \Pi)=\frac{n}{m}\left(\widetilde{X}^{T} \widetilde{X}\right)^{-1}\left(\widetilde{X}^{T} \Omega_{e} \widetilde{X}\right)^{-1}\left(\widetilde{X}^{T} \widetilde{X}\right)^{-1}
$$

since $\Pi \Pi^{T}=\frac{n}{m} I_{m}$. The variance of $\widetilde{\beta}$ is inflated over that of $\hat{\beta}$ through the sketching error on the 'bread' $\left(\widetilde{X}^{T} \widetilde{X}\right)^{-1}$, as well as on the 'meat' because $X^{T} \Omega_{e} X$ is now approximated by $\widetilde{X}^{T} \Pi \Omega_{e} \Pi^{T} \widetilde{X}$. If $e \sim\left(0, \sigma_{e}^{2} I_{n}\right)$ is homoskedastic, then $\widetilde{\beta}$ has variance

$$
\mathbb{V}(\widetilde{\beta} \mid \Pi)=\sigma_{e}^{2} \frac{n}{m}\left(\widetilde{X}^{T} \widetilde{X}\right)^{-1} .
$$


Though $\hat{\beta}$ is the best linear unbiased estimator under homoskedasticity, $\widetilde{\beta}$ may not be best in the class of linear estimators using sketched data.

\subsection{Efficiency of $\widetilde{\beta}$ Under Uniform Sampling}

Suppose we are interested in predicting $y$ at some $x^{0}$. According to the model, $\mathbb{E}\left[y \mid x=x^{0}\right]=$ $\beta^{T} x^{0}$. Feasible predictions are obtained upon replacing $\beta$ with $\hat{\beta}$ and $\widetilde{\beta}$. Since both estimators are unbiased, their respective variance is also the mean-squared prediction error.

Theorem 1 Suppose that $e_{i} \sim\left(0, \sigma_{e}^{2}\right)$ and Assumptions OLS and PI3 hold. Let $\operatorname{MSE}\left(x_{0}^{T} \hat{\beta}\right)$ and $\operatorname{MSE}\left(x_{0}^{T} \widetilde{\beta} \mid \Pi\right)$ be the mean-squared prediction error of $y$ at $x_{0}$ using $\hat{\beta}$ and $\widetilde{\beta}$ conditional on $\Pi$, respectively. Then with probability at least $1-\delta_{\sigma}$, it holds that

$$
\frac{\operatorname{MSE}\left(x_{0}^{T} \widetilde{\beta} \mid \Pi\right)}{\operatorname{MSE}\left(x_{0}^{T} \hat{\beta}\right)} \leq \underbrace{\frac{n}{m}}_{\text {sample size }} \underbrace{\left(\frac{1}{1-\varepsilon_{\sigma}}\right)}_{\text {sketching error }} .
$$

The prediction error has has two components: a sample size effect given by $\frac{n}{m}>1$, and a sketching effect given by $\frac{1}{1-\varepsilon_{\sigma}}>1$. The result arises because under homoskedasticity,

$x_{0}^{T}\left(\widetilde{X}^{T} \widetilde{X}\right)^{-1} x_{0}-x_{0}^{T}\left(X^{T} X\right)^{-1} x_{0}=\frac{n}{m} x_{0}^{T}\left[\left(X^{T} \Pi^{T} \Pi X\right)^{-1}-\left(X^{T} X\right)^{-1}\right] x_{0}+\frac{n-m}{m} x_{0}^{T}\left(X^{T} X\right)^{-1} x_{0}$.

It follows that

$$
\begin{aligned}
\left|\frac{x_{0}^{T} \mathbb{V}(\widetilde{\beta} \mid \Pi) x_{0}-x_{0}^{T} \mathbb{V}(\hat{\beta}) x_{0}}{x_{0}^{T} \mathbb{V}(\hat{\beta}) x_{0}}\right| & =\left|\frac{n}{m} \frac{x_{0}^{T}\left[\left((\Pi X)^{T} \Pi X\right)^{-1}-\left(X^{T} X\right)^{-1}\right] x_{0}}{x_{0}^{T}\left(X^{T} X\right)^{-1} x_{0}}+\frac{n-m}{m}\right| \\
& \leq \frac{n}{m} \frac{\varepsilon_{\sigma}}{1-\varepsilon_{\sigma}}+\frac{n-m}{m} .
\end{aligned}
$$

We will subsequently be interested in the effect of sketching for testing linear restrictions as given by a $K \times 1$ vector $c$. The estimated linear combination $c^{T} \widetilde{\beta}$ has variance $\mathbb{V}\left(c^{T} \widetilde{\beta} \mid \Pi\right)=c^{T} \mathbb{V}(\widetilde{\beta} \mid \Pi) c$. When $c$ is a vector of zeros except in the $k$-th entry, $\operatorname{var}\left(c^{T} \widetilde{\beta} \mid \Pi\right)$ is the variance of $\widetilde{\beta}_{k}$. When $c$ is a vector of ones, $\operatorname{var}\left(c^{T} \widetilde{\beta} \mid \Pi\right)$ is the variance of the sum of estimates. A straightforward generalization of Theorem 1 leads to the following.

Corollary 1 Let c be a known $K \times 1$ vector. Under the Assumptions of Theorem 1, it holds with probability $1-\delta_{\sigma}$ that

$$
\frac{c^{T} \mathbb{V}(\widetilde{\beta} \mid \Pi) c}{c^{T} \mathbb{V}(\hat{\beta}) c} \leq \frac{n}{m}\left(\frac{1}{1-\varepsilon_{\sigma}}\right) .
$$

The relative error is thus primarily determined by the relative sample size. As $m$ is expected to be much smaller than $n$, the efficiency loss is undeniable. 
A lower bound in estimation error can be obtained for embedding matrices $\Phi \in \mathbb{R}^{m \times n}$ satisfying $\|\Phi U\|_{2}^{2} \leq 1+\varepsilon_{\sigma}$. For a sketch size of $m$ rows, define a class of OLS estimators as follows:

$$
\mathcal{B}\left(m, n, \varepsilon_{\sigma}\right):=\left\{\breve{\beta}:=\left((X \Phi)^{T} \Phi X\right)^{-1}(X \Phi)^{T} \Phi y\right\} .
$$

For such $\breve{\beta}$, let $\mathbb{V}(\breve{\beta} \mid \Phi)$ denote its mse for given $\Phi$. Assuming that $e_{i} \sim\left(0, \sigma_{e}^{2}\right)$,

$$
\begin{aligned}
\frac{c^{T} \mathbb{V}(\breve{\beta} \mid \Phi) c}{c^{T} \mathbb{V}(\hat{\beta}) c} & =\frac{m^{-1} \sigma_{e}^{2} c^{T}\left((\Phi X)^{T} \Phi X\right)^{-1} c}{n^{-1} \sigma_{e}^{2} c^{T}\left(X^{T} X\right)^{-1} c}=\frac{n}{m} \frac{c^{T}\left((\Phi X)^{T} \Phi X\right)^{-1} c}{c^{T}\left(X^{T} X\right)^{-1} c} \\
& =\frac{n}{m} \frac{c^{T}\left(V \Sigma U^{T} \Phi^{T} \Phi U \Sigma V^{T}\right)^{-1} c}{c^{T}\left(V \Sigma^{2} V^{T}\right)^{-1} c}=\frac{n}{m} \frac{c^{T} V \Sigma^{-1}\left(U^{T} \Phi^{T} \Phi U\right)^{-1} \Sigma^{-1} V^{T} c}{c^{T} V \Sigma^{-2} V^{T} c} \\
& \geq \frac{n}{m} \sigma_{\min }\left[\left(U^{T} \Phi^{T} \Phi U\right)^{-1}\right] .
\end{aligned}
$$

But by the definition of spectral norm, $\|\Phi U\|_{2}^{2}=\sigma_{\max }^{2}(\Phi U)$ for any $\Phi$. Thus the subspace embedding condition $\|\Phi U\|_{2}^{2} \leq 1+\varepsilon_{\sigma}$ implies $\sigma_{\max }^{2}(\Phi U)=\sigma_{\max }\left((\Phi U)^{T} \Phi U\right) \leq 1+\varepsilon_{\sigma}$, and hence

$$
\sigma_{\min }\left(\left(U^{T} \Phi^{T} \Phi U\right)^{-1}\right) \geq \frac{1}{1+\varepsilon_{\sigma}}
$$

This leads to the following lower bound for $\breve{\beta}$ :

$$
\frac{c^{T} \mathbb{V}(\breve{\beta} \mid \Phi) c}{c^{T} \mathbb{V}(\hat{\beta}) c} \geq \frac{n}{m}\left(\frac{1}{1+\varepsilon_{\sigma}}\right) .
$$

Combining the upper and lower bounds leads to the following:

Theorem 2 Under OLS and PI3, the estimator $\widetilde{\beta}$ with $e_{i} \sim\left(0, \sigma_{e}^{2}\right)$ has mean-squared error relative to the full sample estimator $\hat{\beta}$ bounded by

$$
\frac{n}{m}\left(\frac{1}{1+\varepsilon_{\sigma}}\right) \leq \frac{c^{T} \mathbb{V}(\widetilde{\beta} \mid \Pi) c}{c^{T} \mathbb{V}(\hat{\beta}) c} \leq \frac{n}{m} \frac{1}{1-\varepsilon_{\sigma}} .
$$

These are the upper and lower bounds for uniform sampling when implemented by sampling without replacement.

It is also of interest to know how heteroskedasticity affects the sketching error. Let $\Omega_{e, i i}$ denote the $i$ th diagonal element of $\Omega_{e}$. Under OLS and PI3, it holds with probability at least $1-\delta_{\sigma}$ that

$$
\frac{\operatorname{MSE}\left(x_{0}^{T} \widetilde{\beta} \mid \Pi\right)}{\operatorname{MSE}\left(x_{0}^{T} \hat{\beta}\right)} \leq\left(\frac{\max _{i} \Omega_{e, i i}}{\min _{i} \Omega_{e, i i}}\right)\left(\frac{n}{m}\right) \frac{\left(1+\varepsilon_{\sigma}\right)}{\left(1-\varepsilon_{\sigma}\right)^{2}} .
$$

Hence heteroskedasticity independently interacts with the structure of $\Pi$ to inflate the mean-squared prediction error. The upper and lower bound for $\mathbb{V}\left(c^{T} \widetilde{\beta} \mid \Phi\right)$ are larger than under homoskedasticity by a magnitude that depends on the extent of dispersion in $\Omega_{e, i i}$. A formal result is given in the appendix. 


\subsection{Efficiency of $\widetilde{\beta}$ under Countsketch}

Condition PI.(iii) puts restrictions on $\Pi^{T} \Pi$ and holds for uniform sampling. But the condition does not hold for the countsketch. In its place, we assume the following to obtain a different embedding result for the countsketch:

Assumption CS: For given $\varepsilon_{\Pi}>0$ and for all $U \in \mathbb{R}^{n \times K}$ satisfying $U^{T} U=I_{K}$, there exists an $n \times n$ matrix $A\left(\Omega_{e}, m, n\right)$, which may depend on $\left(\Omega_{e}, m, n\right)$, and a constant $\delta_{\Pi} \in(0,1)$ such that

$$
P\left(\left\|U^{T} \Pi^{T} \Pi \Omega_{e} \Pi^{T} \Pi U-U^{T} A\left(\Omega_{e}, m, n\right) U\right\|_{2} \leq \frac{n}{m} \varepsilon_{\Pi}\right) \geq 1-\delta_{\Pi} .
$$

The conditions for Assumption CS are verified in Appendix B. The Assumption is enough to provide a subspace embedding result for $\Pi^{T} \Pi \Omega_{e} \Pi^{T} \Pi$ because as shown in the Appendix, the following holds for Countsketch,

$$
\begin{aligned}
& \left\|U^{T} \Pi^{T} \Pi \Omega_{e} \Pi^{T} \Pi U-\frac{n}{m} U^{T} \Omega_{e} U\right\|_{2} \\
& \leq\left\|U^{T} \Pi^{T} \Pi \Omega_{e} \Pi^{T} \Pi U-U^{T} A\left(\Omega_{e}, m, n\right) U\right\|_{2}+\left\|U^{T} A\left(\Omega_{e}, m, n\right) U-\frac{n}{m} U^{T} \Omega_{e} U\right\|_{2} \\
& \leq \frac{n}{m}\left[\varepsilon_{\Pi}+\left\|\frac{m}{n} A\left(\Omega_{e}, m, n\right)-\Omega_{e}\right\|_{2}\right]
\end{aligned}
$$

where

$$
A\left(\Omega_{e}, m, n\right)=\Omega_{e}+\frac{1}{m}\left(\operatorname{tr}\left(\Omega_{e}\right) I_{n}-\Omega_{e}\right) .
$$

Hence under OLS, PI2, and CS it holds with probability at least $1-\delta_{\Pi}-\delta_{\sigma}$ that

$$
\frac{\operatorname{MSE}\left(x_{0}^{T} \widetilde{\beta} \mid \Pi\right)}{\operatorname{MSE}\left(x_{0}^{T} \hat{\beta}\right)} \leq\left(\frac{\max _{i} \Omega_{e, i i}}{\min _{i} \Omega_{e, i i}}\right)\left(\frac{n}{m}\right)\left(\frac{1}{1-\varepsilon_{\sigma}}\right) \frac{\left[1+\varepsilon_{\Pi}+\left\|\frac{m}{n} A\left(\Omega_{e}, m, n\right)-\Omega_{e}\right\|_{2}\right]}{\left(1-\varepsilon_{\sigma}\right)} .
$$

The prediction error of the countsketch depends on the quantity $A\left(\Omega_{e}, m, n\right)$. But if $\Omega_{e}=\sigma_{e}^{2} I_{n}$, $\left\|\frac{m}{n} A\left(\Omega_{e}, m, n\right)-\Omega_{e}\right\|_{2}=\sigma_{e}^{2}\left(\frac{m-1}{n}\right)$, which will be negligible if $m / n=o(1)$. Hence to a first approximation, Theorem 1 also holds under the countsketch. This result is of interest since the countsketch is computationally inexpensive.

\section{$5.3 \quad$ Hypothesis Testing}

The statistical implications of sketching in a regression setting have primarily focused on properties of the point estimates. The implications for inference are largely unknown. We analyze the problem from view point of hypothesis testing.

Consider the goal of testing $q$ linear restrictions formulated as $H_{0}: R \beta=r$ where $R$ is a $q \times K$ matrix of restrictions with no unknowns. In this subsection, we further assume that $e_{i} \sim N\left(0, \sigma_{e}^{2}\right)$. 
Under normality, the $F$ test is exact and has the property that at the true value of $\beta=\beta^{0}$,

$$
F_{n}=R\left(\hat{\beta}-\beta_{0}\right)^{T}(\hat{\mathbb{V}}(R \hat{\beta}))^{-1} R\left(\hat{\beta}-\beta_{0}\right) \sim \mathbb{F}_{q, n-d} .
$$

Under the null hypothesis that $\beta_{0}$ is the true value of $\beta, F_{n}$ has a Fisher distribution with $q$ and $n-d$ degrees of freedom. The power of a test given a data of size $n$ against a fixed alternative $\beta_{1} \neq \beta_{0}$ depends on $\mathbb{V}(\hat{\beta})$ only through non-centrality parameter $\phi_{n}{ }_{13}^{13}$ defined in Wallace (1972) as

$$
\phi_{n}=\frac{\left(R \beta_{0}-r\right)^{T} \mathbb{V}(R \hat{\beta})^{-1}(R \beta-r)}{2} .
$$

The non-centrality parameter is increasing in $\left|R \beta_{0}-r\right|$ and the sample size through the variance, but decreasing in $\sigma_{e}^{2}$. In the case of one restriction $(q=1)$,

$$
\phi_{n}=\frac{\left(R \beta_{0}-r\right)^{2}}{\mathbb{V}(R \hat{\beta})}>\frac{\left(R \beta_{0}-r\right)^{2}}{\mathbb{V}(R \widetilde{\beta} \mid \Pi)}=\phi_{m}
$$

This leads to the relative non-centrality

$$
\frac{\phi_{n}}{\phi_{m}}=\frac{\mathbb{V}(R \widetilde{\beta} \mid \Pi)}{\mathbb{V}(R \hat{\beta})} \leq \frac{n}{m} \frac{1}{\left(1-\varepsilon_{\phi}\right)} .
$$

which also has a sample size effect and an effect due to sketching error. The effective size of the subsample from the viewpoint of power can be thought of as $m\left(1-\varepsilon_{\phi}\right)$.

A loss in power is to be expected when $\widetilde{\beta}$ is used. But by how much? Insights can be performed from some back of the envelope calculations. Recall that if $U$ and $V$ are independent $\chi^{2}$ variables with $\nu_{1}$ and $\nu_{2}$ degrees of freedom, $V$ is central and $U$ has non-centrality parameter $\phi$,

$$
\mathbb{E}[F]=\mathbb{E}\left[\frac{\left(U / \nu_{1}\right)}{\left(V / \nu_{2}\right)}\right]=\frac{\nu_{2}\left(\nu_{1}+\phi_{n}\right)}{\nu_{1}\left(\nu_{2}-2\right)} .
$$

In the full sample case, $\nu_{1}=q$ and $\nu_{2}=n-d$ and hence

$$
\mathbb{E}\left[F_{n}\right] \approx \frac{(n-d)\left(q+\phi_{n}\right)}{q(n-d-2)} .
$$

For the subsampled estimator, $\nu_{1}=q$ and $\nu_{2}=m-d$, giving

$$
\mathbb{E}\left[F_{m} \mid \Pi\right] \approx \frac{(m-d)\left(q+\phi_{m}\right)}{q(m-d-2)} .
$$

While $q$ and $\phi_{m}$ affect absolute power, the relative power of testing a hypothesis against a fixed alternative is mainly driven by the relative sample size, $\frac{m}{n}$. However, the power loss from using $\widetilde{\beta}$ to test hypothesis can be made negligible because in a big data setting, we have the luxury of allowing $m$ to be as large as we wish, irrespective of $q$. We will return to the choice of $m$.

\footnotetext{
${ }^{13}$ The definition of non-centrality is not universal, sometimes the factor of two is omitted. See, for example, Cramer (1987) and Rudd (2000).
} 


\section{Econometrically Motivated Refinements}

As more and more data are being collected, sketching continues to be an active area of research. For any sketching scheme, a solution of higher accuracy can be obtained by iteration. The idea is to approximate the deviation from an initial estimate $\Delta=\hat{\beta}-\widetilde{\beta}^{(1)}$ by solving, for example, $\hat{\Delta}^{(1)}=\operatorname{argmin}_{\Delta} \| y-\left(X\left(\widetilde{\beta}^{(1)}+\Delta\right) \|_{2}^{2}\right.$ and update $\widetilde{\beta}^{(2)}=\widetilde{\beta}^{(1)}+\hat{\Delta}^{(1)}$. Pilanci and Wainwright (2016) starts with the observation that since the least squares objective function is $\|y-X \beta\|_{2}^{2}=$ $\|y\|_{2}^{2}+\|X \beta\|_{2}^{2}-2 y^{T} X \beta$, it is possible to sketch the quadratic term $\|X \beta\|_{2}^{2}$ but not the linear term $y^{T} X \beta$. The result is a Hessian sketch of $\beta$, defined as $\left((\Pi X)^{T}(\Pi X)\right)^{-1} X^{T} y$. Wang et al. (2017) suggests that this can be seen as a type of Newton updating with the true Hessian replaced by the sketched Hessian, and the iterative Hessian sketch is also a form of iterative random projection.

In the rest of this section, we consider statistically motivated ways to improve upon $\widetilde{\beta}$. Subsection 6.1 considers pooling estimates from multiple sketches. Subsection 6.2 suggests an $m$ that is motivated by hypothesis testing.

\subsection{Combining Sketches}

The main result of the previous section is that the least squares estimates using sketched data has two errors, one due to a smaller sample size, and one due to sketching. The efficiency loss is hardly surprising, and there are different ways to improve upon it. Dhillon et al. (2013) proposes a twostage algorithm that uses $m$ rows of $(y, X)$ to obtain an initial estimate of $\Sigma_{X X}$ and $\Sigma_{X y}$. In the second stage, the remaining rows are used to estimate the bias of the first stage estimator. The final

estimate is a weighted average of the two estimates. An error bound of $O\left(\frac{\sqrt{K}}{\sqrt{n}}\right)$ is obtained. This bound is independent of the amount of subsampling provided $m>O(\sqrt{K / n})$. Chen et al. (2016) suggests to choose sample indices from an importance sampling distribution that is proportional to a sampling score computed from the data. They show that the optimal $p_{i}$ depends on whether minimizing mean-squared error of $\widetilde{\beta}$ or of $X \widetilde{\beta}$ is the goal, though $\mathbb{E}\left[e_{i}^{2}\right]$ plays a role in both objectives.

The sample size effect is to be expected, and is the cost we pay for not being able to use the full data. But if it is computationally simple to create a sample of size $m$, the possibility arises that we can better exploit information in the data without hitting the computation bottleneck by generating many subsamples and subsequently pool estimates constructed from the subsamples. Breiman (1999) explored an idea known as pasting bites that, when applied to regressions, would repeatedly form training samples of size $m$ by random sampling from the original data, then make prediction by fitting the model to the training data. The final prediction is the average of the predictions. Similar ideas are considered in Chawla et al. (2004) and Christmann et al. (2007). Also related is distributed computing which takes advantage of many nodes in the computing cluster. 
Typically, each machine only sees a subsample of the full data and the parameter of interest is updated. Heince et al. (2016) studies a situation when the data are distrubuted across workers according to features of $X$ rather than the sample and show that their DUAL LOCO algorithm has bounded approximation error that depends only weakly on the number of workers.

Consider $\widetilde{\beta}_{1}, \ldots, \widetilde{\beta}_{J}$ computed from $J$ subsamples each of size $K$. As mentioned above, uniform sampling with too few rows is potentially vulnerable to omitting influential observations. Computing multiple sketches also provides the user with an opportunity to check the rank across sketches. Let $\widetilde{t}_{j}=\frac{\widetilde{\beta}_{j}-\beta_{0}}{\operatorname{se}\left(\widetilde{\beta}_{j}\right)}$ be the $t$ test from sketch $j$. For a given $m$, we consider sampling without replacement and $J$ is then at most $n / m$. Define the average quantities

$$
\begin{aligned}
\bar{\beta} & =\frac{1}{J} \sum_{j=1}^{J} \widetilde{\beta}_{j}, & \operatorname{se}(\bar{\beta}) & =\sqrt{\frac{1}{J(J-1)} \sum_{j=1}^{J}\left[\operatorname{se}\left(\widetilde{\beta}_{j}\right)\right]^{2},} \\
\bar{t}_{2} & =\frac{1}{J} \sum_{j=1}^{J} \widetilde{t}_{j}, & \operatorname{se}\left(\bar{t}_{2}\right) & =\sqrt{\frac{1}{J-1} \sum_{j=1}^{J}\left(\widetilde{t}_{j}-\bar{t}_{2}\right)^{2}} .
\end{aligned}
$$

Strictly speaking, pooling requires that subsamples are non-overlapping and observations are independent across different subsamples. Assuming independence across $j$, the pooled estimator $\bar{\beta}$ has $\operatorname{var}(\bar{\beta})=\frac{1}{J^{2}} \sum_{j=1}^{J} \operatorname{var}\left(\widetilde{\beta}_{j}\right)$. Thus, $\operatorname{se}(\bar{\beta}) \approx \sqrt{\frac{1}{J^{2}} \sum_{j=1}^{J}\left[\operatorname{se}\left(\widetilde{\beta}_{j}\right)\right]^{2}} \geq \frac{1}{\sqrt{J}}\left[\frac{1}{J} \sum_{j=1}^{J} \operatorname{se}\left(\widetilde{\beta}_{j}\right)\right]$ because of Jansen's inequality. Our estimator for the standard error $\bar{\beta}$ uses $J-1$ in the denominator to allow for a correction when $J$ is relatively small.

Consider two pooled $t$ statistics:

$$
\begin{aligned}
\bar{T}_{1} & =\frac{\bar{\beta}-\beta_{0}}{s e(\bar{\beta})} \\
\bar{T}_{2} & =\sqrt{J} \frac{\bar{t}_{2}}{s e\left(\bar{t}_{2}\right)} .
\end{aligned}
$$

Critical values from the standard normal distribution can be used for $\bar{T}_{1}$. For example, for the 5\%-level test, we reject $H_{0}$ if $\left|\bar{T}_{1}\right|>1.96$. For $\bar{T}_{2}$, we recommend using critical values from the $t$ distribution with $J-1$ degrees of freedom. For example, for the $5 \%$-level test, we reject $H_{0}$ if $\left|\bar{T}_{2}\right|>2.776$ for $J=5$.

\section{Assumption PI-Avg:}

(i) $\left(\Pi_{1}, \ldots, \Pi_{J}\right)$ is independent of $e$;

(ii) for all $j, k$ such that $j \neq k, \Pi_{j} \Pi_{j}^{T}=\frac{n}{m} I_{m}$ and $\Pi_{j} \Pi_{k}^{T}=O_{m}$ where $O_{m}$ is an $m \times m$ matrix of zeros. 
(iii) for given singular value distortion parameter $\varepsilon_{\sigma} \in(0,1)$, there exists failure parameter $\delta_{\sigma} \in$ $(0,1)$ such that $P\left(\left|1-\sigma_{k}^{2}\left(\Pi_{j} U\right)\right| \leq \varepsilon_{\sigma}\right.$ for all $k=1, \ldots K$ and for all $\left.j=1, \ldots, J\right) \geq 1-\delta_{\sigma}$.

Condition (ii) is crucial; it is satisfied, for example, if $\Pi_{1}, \ldots, \Pi_{J}$ are non-overlapping subsamples and each of them is sampled uniformly without replacement. Assumptions OLS and PI-Avg (i) and (ii) along with the homoskedastic error assumption ensure that

$$
c^{T} \mathbb{V}\left(\bar{\beta} \mid \Pi_{1}, \ldots, \Pi_{J}\right) c=\sigma_{e}^{2} \frac{n}{m J^{2}} \sum_{j=1}^{J} c^{T}\left(X^{T} \Pi_{j}^{T} \Pi_{j} X\right)^{-1} c .
$$

Condition (iii) is equivalent to the statement that $1-\varepsilon_{\sigma} \leq \sigma_{k}\left(U^{T} \Pi_{j}^{T} \Pi_{j} U\right) \leq 1+\varepsilon_{\sigma} \quad \forall k=$ $1, \ldots, K, \forall j=1, \ldots, J$.

Theorem 3 Consider $J$ independent sketches obtained by uniform sampling. Suppose that $e_{i} \sim$ $\left(0, \sigma_{e}^{2}\right)$, Assumptions $O L S$ and PI-Avg hold. Then with probability at leat $1-\delta_{\sigma}$, the mean squared error of $c^{T} \bar{\beta}$ conditional on $\left(\Pi_{1}, \ldots, \Pi_{J}\right)$ satisifies

$$
\frac{c^{T} \mathbb{V}\left(\breve{\beta} \mid \Pi_{1}, \ldots, \Pi_{J}\right) c}{c^{T} \mathbb{V}(\hat{\beta}) c} \leq \frac{n}{m J} \frac{1}{\left(1-\varepsilon_{\sigma}\right)} .
$$

The significance of the Theorem is that by choice of $m$ and $J$, the pooled estimator can be almost as efficient as the full sample estimator. If we set $J=1$, the theorem reduces to Theorem 1 .

We use a small Monte Carlo experiment to assess the effectiveness of combining statistics computed from different sketches. The data are generated as $y=X \beta+e$ where $e$ is normally distributed, and $X$ is drawn from a non-normal distribution. In matlab, we have $\mathrm{X}=\operatorname{PEARSRND}(0,1,1,5, \mathrm{~N}, \mathrm{~K})$. With $n=1 e 6$, we consider different values of $m$ and $J$. Most of our results above were derived for uniform sampling, so it is also of interest to evaluate the properties of the $\widetilde{\beta}$ using data sketched by ПIs that do not satisfy PI.(iii). Four sketching schemes are considered: uniform sampling without replacement labeled as Rs1, SRHT, the countsketch labeled CS, and leverage score sampling, labeled LEV. It should be mentioned that results for SHRT and LEV took significantly longer time to compute than RS1 and CS.

The top panel of Table 2 reports results for $\hat{\beta}_{3}$ when $K=3$. All sampling schemes precisely estimate $\beta_{3}$ whose true value is one. The standard error is larger the smaller is $m$, which is the sample size effect. But averaging $\widetilde{\beta}_{j}$ over $j$ reduces variability. The LEV is slightly more efficient. The size of the $t$ test for $\beta_{3}=1.0$ is accurate, and the power of the test against $\beta_{3}<1$ when $\beta_{3}=0.98$ is increasing in the amount of total information used. Combining $J$ sketches of size $m$ generally gives a more powerful test than a test based on a sketch size of $m J$. The bottom panel of Table 2 reports for $K=9$, focusing on uniform sampling without replacement and the countsketch. 
The results are similar to those for $K=3$. The main point to highlight is that while there is a sample size effect from sketching, it can be alleviated by pooling across sketches.

\subsection{The Choice of $m$}

The JL Lemma shows that $m=O\left(\log d \epsilon^{-2}\right)$ rows are needed for $d$ vectors from $\mathbb{R}^{n}$ to be embedded into an $m$ dimensional subspace. A rough and ready guide for embedding a $d$ dimensional subspace is $m=\Omega\left(d \log d \epsilon^{-2}\right)$. This is indeed the generic condition given in, for example, Sarlos (2006), though more can be said for certain $\Pi s{ }^{14}$ Notably, these desired $m$ for random projections depend only on $d$ but not on $n$.

As shown in Boutidis and Gittens (2013, Lemma 4.3), subspace embedding by uniform sampling without replacement requires that

$$
m \geq 6 \varepsilon_{\sigma}^{-2} n \ell_{\max } \log \left(2 J \cdot K / \delta_{\sigma}\right) .
$$

where $\ell_{\max }=\max _{i} \ell_{i}$ is the maximum leverage score, also known as coherence. When coherence is large, the information in the data is not well spread out, and more rows are required for uniform sampling to provide subspace embedding. Hence unlike random projections, the desired $m$ for uniform sampling is not data oblivious.

But while this choice of $m$ is algorithmically desirable, statistical analysis often cares about the variability of the estimates in repeated sampling, and a larger $m$ is always desirable for $\mathbb{V}(\widetilde{\beta})$. The question arises as to whether $m$ can be designed to take both algorithmic and statistical considerations into account. We suggest two ways to fine-tune the algorithmic condition. Now

$$
\begin{aligned}
\ell_{i}=\left\|U_{(i)}\right\|^{2} & =X_{(i)}^{T}\left(X^{T} X\right)^{-1} X_{(i)}=\frac{1}{n} X_{(i)}^{T} S_{X}^{-1} X_{(i)} \\
& \leq \sigma_{1}\left(S_{X}^{-1}\right) \frac{1}{n}\left\|X_{(i)}\right\|_{2}^{2}=\sigma_{K}^{-1}\left(S_{X}\right) \frac{1}{n}\left\|X_{(i)}\right\|_{2}^{2} .
\end{aligned}
$$

where $\sigma_{K}\left(S_{X}\right)$ is the minimum eigenvalue of $S_{X}=n^{-1} X^{T} X, X_{(i)}^{T}$ denotes the $i$-th row of $X$, and $X_{(i, j)}$ the $(i, j)$ element of $X$. But

$$
\left\|X_{(i)}\right\|_{2}^{2}=\sum_{j=1}^{K}\left[X_{(i, j)}\right]^{2} \leq K \cdot \max _{j=1, \ldots, K}\left[X_{(i, j)}\right]^{2}=K \cdot X_{\max }^{2}
$$

where $X_{\max }=\max _{i=1, \ldots, n} \max _{j=1, \ldots, d}\left|X_{(i, j)}\right|$. This implies $n \cdot \ell_{\max } \leq \sigma_{K}^{-1}\left(S_{X}\right) \cdot K \cdot X_{\max }^{2} \cdot$ Recalling that $p_{i}=\frac{\ell_{i}}{K}$ defines the importance sampling distribution, we can now restate the algorithmic condition for $m$ when $J=1$ as

$$
m=\Omega\left(n K \log (K) \cdot p_{\max }\right) \quad \text { where } \quad p_{\max } \leq \frac{\sigma_{K}^{-1}\left(S_{X}\right) X_{\max }^{2}}{n} .
$$

\footnotetext{
${ }^{14}$ The result for SRHT is proved in Lemma 4.1 of Boutidis and Gittens (2013). The result for count sketch is from Theorem 2 of Nelson and Nguyen (2013a).
} 
It remains to relate $X_{\max }$ with quantities of statistical interest.

\section{Assumption M:}

a. $\sigma_{K}\left(S_{X}\right)$ is bounded below by $c_{X}$ with probability approaching one as $n \rightarrow \infty$;

b. $\mathbb{E}\left[\left|X_{(i, j)}\right|^{r}\right] \leq C_{X}$ for some $C_{X}$ and some $r \geq 2$.

Condition (a) is a standard identification condition for $S_{X}$ to be positive definite so that it will converge in probability to $\mathbb{E}\left[X_{(i)} X_{(i)}^{T}\right]$. Condition (b) requires the existence of $r$ moments so as to bound extreme values $\sqrt{15}$ If the condition holds,

$$
X_{\max }=o_{p}\left((n K)^{1 / r}\right) .
$$

Proposition 1 Suppose that Assumption M holds. A deterministic rule for sketched linear regressions by uniform sampling is

$$
\begin{cases}m_{1}=\Omega\left((n K)^{1+2 / r} \log K / n\right) & \text { if } \quad r<\infty \\ m_{1}=\Omega(K \log (n K)) & \text { if } \quad \mathbb{E}\left[\exp \left(t X_{(i, j)}\right)\right] \leq C_{X} \quad \text { holds additionally. }\end{cases}
$$

Proposition 1 can be understood as follows. Suppose that $r=6$ moments are known to exist. Proposition 1 suggests a sketch of $m_{1}=\Omega\left(\log K(n K)^{4 / 3} / n\right)$ rows which will generally be larger than $\Omega(K \log K)$, which is the sketch size suggested for data with thin tails. For such data, the moment generating function is uniformly bounded and $\mathbb{E}\left[\exp \left(t X_{(i, j)}\right)\right] \leq C_{X}$ for some constant $C_{X}$ and some $t>0$ so that $X_{\max }=o_{p}(\log (n K))$. In both cases, the desired $m$ increases with the row sample size $n$, the number of regressors $K$, as well as $n \cdot K$, which is the number of data points in the regressor matrix $X$. This contrasts with the algorithmic condition for $m$ which does not depend on $n$.

To use Proposition 1, we can either (i) fix $r$ to determine $m_{1}$ or (ii) target 'observations-perregressor ratio'. As an example, suppose $n=1 e 7$ and $K=10$. If $r=6$, Proposition 1 suggests to sample $m_{1}=10,687$ rows, implying $\frac{m_{1}}{K} \approx 1000$. If instead we fix $\frac{m}{K}$ at 100 and uniformly sample $m_{1}=1000$ rows, we must be ready to defend the existence of $r=\frac{2 \log (n K)}{\log \left(\frac{m}{K}\right)-\log (\log K)} \approx 10$ moments. There is a clear trade-off between $m_{1}$ and $r$.

Though $m_{1}$ depends on $n$, it is still a deterministic rule. To obtain a rule that is data dependent, consider again $c^{T} \widetilde{\beta}$, where $c$ is a $K \times 1$ vector, and assume that $e_{i} \sim N\left(0, \sigma^{2}\right)$ so that $\operatorname{var}(\widetilde{\beta})=$ $\frac{n}{m} \sigma_{e}^{2}\left(\widetilde{X}^{T} \widetilde{X}\right)^{-1}$ where $\beta_{0}$ is the true (unknown) value of $\beta$. Define

$$
\tau_{0}(m)=\frac{c^{T}\left(\widetilde{\beta}-\beta_{0}\right)}{\operatorname{SE}\left(c^{T} \widetilde{\beta}\right)}=\frac{c^{T}\left(\widetilde{\beta}-\beta^{0}\right)+c^{T}\left(\beta^{0}-\beta_{0}\right)}{\operatorname{SE}\left(c^{T} \widetilde{\beta}\right)} .
$$

\footnotetext{
${ }^{15}$ Similar conditions are used to obtain results for the hat matrix. See, for example, Section 6.23 of Hansen (2019)'s online textbook.
} 
It holds that $P_{\beta_{0}}\left(\tau_{0}>z \mid \Pi, X_{1}, \ldots, X_{n}\right)=\Phi(-z)$ for some $z$, where $\Phi(\cdot)$ is the cdf of the standard normal distribution. Now consider a one-sided test $\tau_{1}$ based on $\widetilde{\beta}$ against an alternative, say, $\beta^{0}$. The test $\tau_{1}$ is related to $\tau_{0}$ by

$$
\tau_{1}(m)=\frac{c^{T}\left(\widetilde{\beta}-\beta_{0}\right)}{\operatorname{SE}\left(c^{T} \widetilde{\beta}\right)}-\frac{c^{T}\left(\beta^{0}-\beta_{0}\right)}{\operatorname{SE}\left(c^{T} \widetilde{\beta}\right)}=\tau_{0}(m)+\tau_{2}(m) .
$$

The power of $\tau_{1}$ at nominal size $\alpha$ is then

$$
P_{\beta_{0}}\left(\tau_{0}+\tau_{2}>\Phi_{(1-\alpha)}^{-1} \mid \Pi, X_{1}, \ldots, X_{n}\right)=\Phi\left(-\Phi_{(1-\alpha)}^{-1}+\tau_{2}\right) \equiv \gamma
$$

Define

$$
S(\alpha, \gamma)=\Phi_{\gamma}^{-1}+\Phi_{1-\alpha}^{-1}
$$

Common values of $\alpha$ and $\gamma$ give the following:

\begin{tabular}{l|ccccc}
\multicolumn{7}{c|}{ Selected values of $S(\alpha, \gamma)$} \\
\hline$\alpha$ & 0.500 & 0.600 & 0.700 & 0.800 & 0.900 \\
\hline 0.010 & 2.326 & 2.580 & 2.851 & 3.168 & 3.608 \\
0.050 & 1.645 & 1.898 & 2.169 & 2.486 & 2.926 \\
0.100 & 1.282 & 1.535 & 1.806 & 2.123 & 2.563 \\
\hline
\end{tabular}

Proposition 2 Suppose that $e_{i} \sim N\left(0, \sigma_{e}^{2}\right)$ and the Assumptions of Theorem 1 hold. Let $\bar{\gamma}$ be the target power of a one-sided test $\tau_{1}$ and $\bar{\alpha}$ be the nominal size of the test.

- Let $\widetilde{\beta}$ be obtained from a sketch of size $m_{1}$. For a given effect size of $\beta^{0}-\beta_{0}$, a data dependent 'inference conscious' sketch size is

$$
m_{2}\left(m_{1}\right)=S^{2}(\bar{\alpha}, \bar{\gamma}) \frac{m_{1} \operatorname{var}\left(c^{T} \widetilde{\beta}\right)}{\left[c^{T}\left(\beta^{0}-\beta_{0}\right)\right]^{2}}=m_{1} \frac{S^{2}(\bar{\alpha}, \bar{\gamma})}{\tau_{2}^{2}\left(m_{1}\right)} .
$$

- For a pre-specified $\tau_{2}(\infty)$, a data oblivious 'inference conscious' sketch size is

$$
m_{3}=n \frac{S^{2}(\bar{\alpha}, \bar{\gamma})}{\tau_{2}^{2}(\infty)}
$$

Inference considerations suggests to adjust $m_{1}$ by a factor that depends on $S(\bar{\alpha}, \bar{\gamma})$ and $\tau_{2}$. For instance, when $\bar{\gamma} \geq 0.8$ and/or $\bar{\alpha} \leq 0.01, m_{1}$ will be adjusted upwards when the $\tau_{2}$ is less than two. The precise adjustment depends on the choice of $\tau_{2}$.

The proposed $m_{2}$ in Part (i) requires an estimate of $\operatorname{vaR}\left(c^{T} \widetilde{\beta}\right)$ from a preliminary sketch. Table 3 provides an illustration for one draw of simulated data with $n=1 e 7$ and $K=10$. We consider three values of $\sigma_{e}, \bar{\gamma}$, as well as different effect size $\beta_{1}^{0}-\beta_{10}$. Assuming $r=10$ and $\sigma_{e}=0.5$, an effect 
size of 0.025 gives an $m_{1}$ of roughly 1000. Using a sketch of this size to obtain an estimate of $\tau_{2}$ will almost hit the target power of 0.5 . However, a target power of 0.9 would require $m_{2}=3,759$, almost four times as many rows as a target power of 0.5. The larger is $\sigma_{e}$, the less precise is $\widetilde{\beta}$ for a given $m$, and more rows are needed. It is then up to the user how to trade of computation cost and power of the test.

The proposed $m_{3}$ in Part (ii) is motivatead by the fact that setting $m_{1}$ to $n$ gives $m_{2}(n)=$ $n \frac{S^{2}(\bar{\alpha}, \bar{\gamma})}{\tau_{2}^{2}(n)}$. Though computation of $\tau_{2}(n)$ is infeasible, $\tau_{2}(n)$ is asymptotically normal as $n \rightarrow \infty$. Now if the full sample $t$-statistic cannot reject the null hypothesis, a test based on sketched data will unlikely reject the null hypothesis. But when full sample $t$ statistic is expected to be relatively large (say, 5), the result can be used in conjunction with $S(\bar{\alpha}, \bar{\gamma})$ to give $m_{3}$. Say if $S(\bar{\alpha}, \bar{\gamma})$ is 2 , $m_{3}=(2 / 5)^{2} n$. This allows us to gauge the sample size effect since $\frac{n}{m}=\frac{\tau_{2}^{2}(\infty)}{S^{2}(\bar{\alpha}, \bar{\gamma})}$. Note that $m_{3}$ only requires the choice of $\bar{\alpha}, \bar{\gamma}$, and $\tau_{2}(\infty)$ which, unlike $m_{2}$, can be computed without a preliminary sketch.

Though Propositions 1 and 2 were derived for uniform sampling, they can still be used for other choice of $\Pi$. The one exception in which some caution is warranted is the countsketch. The rule given for the countsketch in Nelson and Nguyen (2013a, Theorem 5) of $m \geq \varepsilon^{-2} K(K+1) \delta^{-1}$. Though such an $m$ is data oblivious, it is generally larger than the rule given by Boutidis and Gittens (2013) for uniform sampling. The larger $m$ required for countsketch can be seen as a cost of specifying a sparse $\Pi$. Thus, one might want to first use a small $r$ to obtain a conservative $m_{1}$ for the countsketch. One can then use Proposition 2 to obtain an 'inference conscious' guide.

To illustrate how to use $m_{1}, m_{2}$ and $m_{3}$, we consider Belenzon et al. (2017) which studies firms' performance from naming the company after its owners, a phenomenon known as eponymy. The parameter of interest is $\alpha_{1}$ in a 'return on assets' regression

$$
\operatorname{roa}_{i t}=\alpha_{0}+\alpha_{1} \text { eponymous }_{i t}+Z_{i t}^{T} \beta+\eta_{i}+\tau_{t}+c_{i}+\epsilon_{i t} .
$$

The coefficient gives the effect of the eponymous dummy after controlling for time varying firm specific variables $Z_{i t}$, SIC dummies $\eta_{i}$, country dummies $c_{i}$, and year dummies $\tau_{t}$. The panel of data includes 1.8 million companies from 2002-2012, but we only use data for one year. An interesting aspect of this regression is that even in the full sample with $n=562160$, some dummies are sparse while others are collinear, giving an effective number of $K=423$ regressors. We will focus on the four covariates: the indicator variable for being eponymous, the log of assets, the $\log$ number of shareholders, and equity dispersion.

Given the values of $(n, K)$ for this data, any assumed value of $r$ less than 8 would give an $m_{1}$ larger than $n$ which is not sensible ${ }^{16}$ This immediately restricts us to $r \geq 6$. As point of reference,

\footnotetext{
${ }^{16}$ This is based on $m_{1}=(n K)^{1+2 / r} \log K / n$. A smaller $r$ is admissible if $m_{1}=c_{1}(n K)^{1+2 / r} \log K / n$ for some
} 
$\left(r, m_{1}\right)=(8,317657)$ and $\left(r, m_{1}\right)=(15,33476)$. The smallest $m_{1}$ is obtained by assuming that the data have thin tails, resulting in $m_{1}=K \log (n K)=8158$.

Table 4 presents the estimation results for several values of $m$. The top panel presents results for uniform sampling. Note that more than 50 covariates for uniform sampling are omitted due to colinearity, even with a relatively large sketch size. The first column shows the full sample estimates for comparison. Column (2) shows that the point estimates given by the smallest sketch with $m_{1}=8158$ are not too different from those in column (1), but the precision estimates are much worse. To solve this problem, we compute $m_{2}\left(m_{1}\right)$ by plugging in the $t$ statistic for equity dispersion (ie. $\hat{\tau}_{0}=0.67$ ) as $\tau_{2}$. This gives an $m_{2}$ of 112358. A similar sketch size can be obtained by assuming $r=10$ for $m_{1}$, or by plugging in $\tau_{2}(\infty)=5$ for $m_{3}$. As seen from Table 4 , the point estimates of all sketches are similar, but the inference conscious sketches are larger in size and give larger test statistics.

The bottom panel of Table 4 presents results for the countsketch. Compared to uniform sampling in the top panel, only one or two covariates are now dropped. Though the estimate of $\alpha_{1}$ is almost almost identical to the one for the full sample and for uniform sampling, the estimated coefficient for equity dispersion is somewhat different. This might be due to the fact that uniform sampling drops much more covariates than countsketch.

\section{Concluding Remarks}

This paper provides an gentle introduction to sketching and studies its implications for prediction and inference using a linear model. Sample codes for constructing the sketches are avaialble in MATLAB, R, and STATA. Our main findings are as follows:

1. Sketches incur an approximation error that is small relative to the sample size effect.

2. For speed and parallelization, it is best to use countsketch.

3. For simple implementation, it is best to use uniform sampling.

4. For improved estimates, it is best to average over multiple sketches.

5. Statistical analysis may require larger sketch size than what is algorithmically desirable. We propose two inference conscious rules for the sketch size.

Sketching has also drawn attention of statisticians in recent years. Ahfock et al. (2017) provides an inferential framework to obtain distributional results for a large class of sketched estimators. Geppert, Ickstadt, Munteanu, Quedenfeld and Sohler (2017) considers random projections in constant $0<c_{1}<1$. We limit our attention to $c_{1}=1$. 
Bayesian regressions and provides sufficient conditions for a Gaussian likelihood based on sketched data to have an error of $1+O(\epsilon)$ in terms of $L_{2}$ Wasserstein distance. In the design of experiments literature, the goal is to reveal as much information as possible given a fixed budget 17 Since sketching is about forming random samples, it is natural to incorporate the principles in design of experiments. Wang, Zhu and Ma (2018) considers the design of subsamples for logistic regressions. $A$-optimality and practical considerations suggest to use $p_{i}=\frac{\left|\hat{e}_{i}\right|\left\|x_{i}\right\|}{\sum_{i}\left|\hat{e}_{i}\right|\left\|x_{i}\right\|}$, which may be understood as score based sampling. Wang et al. (2019) considers the homoskedastic normal linear regression model. The principle of $D$-optimality suggests to recursively selecting data according to extreme values of covariances. The algorithm is suited for distributed storage and parallel computing.

Though analysis of the linear regression is an absolute first step in understanding the use and implications of sketching, the real benefits are expected to be in estimation problems that are complex. For example, Deaton and Ng (1998) uses 'binning methods' and uniform sampling to speed up estimation of non-parametric average derivatives. Portnoy and Koenker (1997) uses a fast interior point method for quantile regression by preprocessing a random subsample of data to reduce the effective sample size. More generally, if the full-sample Hessian matrix is difficult to estimate, one can consider an approximation of it by subsampling. This paper provides a new perspective to evaluating the effectiveness of matrix approximations and aims to better understand their implications for inference.

While using sketches to overcome the computation burden is a step forward, sometimes we need more than a basic sketch. We have been silent about how to deal with data that are dependent over time or across space, such as due to network effects. We may want our sketch to preserve, say, the size distribution of firms in the original data. The sampling algorithms considered in this review must then satisfy additional conditions. When the data have a probabilistic structure, having more data is not always desirable, Boivin and Ng (2006). While discipline-specific problems require discipline-specific input, there is also a lot to learn from what has already been done in other literatures. Cross-disciplinary work is a promising path towards efficient handling of large volumes of data.

\footnotetext{
${ }^{17} \mathrm{~A}$ criterion that uses the trace norm for ordering matrices is $A$-optimality. A criterion that uses the determinant to order matrices is a $D$-optimal design.
} 


\section{Appendices}

\section{A Proof of Lemma 3}

Proof of Lemma 3 By an orthogonal decomposition of the least squares residuals,

$$
\begin{aligned}
\|y-X \widetilde{\beta}\|_{2}^{2} & =\|y-X \hat{\beta}\|_{2}^{2}+\|X \widetilde{\beta}-X \hat{\beta}\|_{2}^{2} \\
& =\|y-U \hat{\theta}\|_{2}^{2}+\|U(\widetilde{\theta}-\hat{\theta})\|_{2}^{2} \\
& =\widehat{\operatorname{SSR}}^{2}+\|U(\widetilde{\theta}-\hat{\theta})\| \\
& =\widehat{\operatorname{SSR}}^{2}+\|\widetilde{\theta}-\hat{\theta}\|_{2},
\end{aligned}
$$

where

$$
\begin{aligned}
\|\widetilde{\theta}-\hat{\theta}\|_{2} & =\left\|(\Pi U)^{T} \Pi U(\widetilde{\theta}-\hat{\theta})+(\widetilde{\theta}-\hat{\theta})+(\Pi U)^{T} \Pi U(\hat{\theta}-\widetilde{\theta})\right\|_{2} \\
& \leq\left\|(\Pi U)^{T} \Pi U(\widetilde{\theta}-\hat{\theta})\right\|_{2}+\left\|(\Pi U)^{T} \Pi U(\hat{\theta}-\widetilde{\theta})-(\hat{\theta}-\widetilde{\theta})\right\|_{2} \\
& \leq\left\|(\Pi U)^{T} \Pi U(\widetilde{\theta}-\hat{\theta})\right\|_{2}+\left\|(\Pi U)^{T} \Pi U-I_{d}\right\|_{2}\|(\widetilde{\theta}-\hat{\theta})\|_{2} \\
& \leq\left\|(\Pi U)^{T}(\Pi U)(\widetilde{\theta}-\hat{\theta})\right\|_{2}+\frac{1}{\sqrt{2}}\|\widetilde{\theta}-\hat{\theta}\|_{2} \\
& \leq \sqrt{2}\left\|(\Pi U)^{T} \Pi U(\widetilde{\theta}-\hat{\theta})\right\|_{2}
\end{aligned}
$$

by triangle inequality, Cauchy-Schwarz inequality, condition (8a), and rearranging terms. Now the normal equations implies $(\Pi U)^{T}(\Pi U) \widetilde{\theta}=(\Pi U)^{T} \Pi(y-X \widetilde{\beta})$. Hence

$$
\begin{aligned}
\|\widetilde{\theta}-\hat{\theta}\|_{2} & \leq \sqrt{2}\left\|(\Pi U)^{T} \Pi(y-U \hat{\theta})\right\|_{2} \\
& \leq \sqrt{\epsilon_{0}} \widehat{\mathrm{SSR}}
\end{aligned}
$$

by condition $(8 \mathrm{~b})$ and for some failure probability $\delta_{0}$. It follows from $(16)$ that $\widetilde{\mathrm{SSR}}^{2} \leq\left(1+\epsilon_{2}\right) \widehat{\mathrm{SSR}}^{2}$ holds with probability $1-\delta_{2}$ where $\epsilon_{2}=\epsilon_{0}^{2}$ and $\delta_{2}<2 \delta_{0}$. This probability can be made higher with suitable choice of $\epsilon_{0}$ and $m$, which can be controlled by the researcher.

Proof of Theorem 3 Note that

$$
c^{T}(\bar{\beta}-\beta)=\frac{1}{J} \sum_{j=1}^{J} c^{T}\left(X^{T} \Pi_{j}^{T} \Pi_{j} X\right)^{-1}\left(X^{T} \Pi_{j}^{T} \Pi_{j} e\right) .
$$

Thus,

$$
E\left[c^{T}(\bar{\beta}-\beta) \mid \Pi_{1}, \ldots, \Pi_{J}\right]=\frac{1}{J} \sum_{j=1}^{J} c^{T}\left(X^{T} \Pi^{T} \Pi X\right)^{-1}\left(X^{T} \Pi^{T} \Pi E\left[e \mid \Pi_{1}, \ldots, \Pi_{J}\right]\right)=0 .
$$


Write

$$
\begin{aligned}
& E\left[\left\{c^{T}(\bar{\beta}-\beta)\right\}^{2} \mid \Pi_{1}, \ldots, \Pi_{J}\right] \\
& =\frac{1}{J^{2}} \sum_{j=1}^{J} \sum_{k=1}^{J} c^{T}\left(X^{T} \Pi_{j}^{T} \Pi_{j} X\right)^{-1}\left(X^{T} \Pi_{j}^{T} \Pi_{j} E\left[e e^{T} \mid \Pi_{1}, \ldots, \Pi_{J}\right] \Pi_{k}^{T} \Pi_{k} X\right)\left(X^{T} \Pi_{k}^{T} \Pi_{k} X\right)^{-1} c \\
& =\sigma_{e}^{2} \frac{1}{J^{2}} \sum_{j=1}^{J} c^{T}\left(X^{T} \Pi_{j}^{T} \Pi_{j} X\right)^{-1}\left(X^{T} \Pi_{j}^{T} \Pi_{j} \Pi_{j}^{T} \Pi_{j} X\right)\left(X^{T} \Pi_{j}^{T} \Pi_{j} X\right)^{-1} c \\
& +\sigma_{e}^{2} \frac{1}{J^{2}} \sum_{j=1}^{J} \sum_{k=1, k \neq J}^{J} c^{T}\left(X^{T} \Pi_{j}^{T} \Pi_{j} X\right)^{-1}\left(X^{T} \Pi_{j}^{T} \Pi_{j} \Pi_{k}^{T} \Pi_{k} X\right)\left(X^{T} \Pi_{k}^{T} \Pi_{k} X\right)^{-1} c \\
& =\sigma_{e}^{2} \frac{n}{m J^{2}} \sum_{j=1}^{J} c^{T}\left(X^{T} \Pi_{j}^{T} \Pi_{j} X\right)^{-1} c .
\end{aligned}
$$

Then, the desired result is obtained by arguments identical to those used in proving Theorem 1 . In particular, we can show that

$$
\frac{n}{m} \frac{c^{T}\left(X^{T} \Pi_{j}^{T} \Pi_{j} X\right)^{-1} c}{c^{T}\left(X^{T} X\right)^{-1} c} \leq \frac{n}{m} \frac{1}{\left(1-\varepsilon_{\sigma}\right)}
$$

jointly for all $j=1, \ldots, J$ with probability at least $1-\delta_{\sigma}$. Q.E.D.

\section{B Verification of Assumption CS for Countsketch}

In this part of the appendix, we verify Assumption CS for the countsketch. Here, we use $d$ to denote the column dimension of $\Pi$.

Lemma 5 Let $\Pi \in \mathbb{R}^{n \times d}$ be a random matrix such that (i) the $(i, j)$ element $\Pi_{i j}$ of $\Pi$ is $\Pi_{i j}=\delta_{i j} \sigma_{i j}$, where $\sigma_{i j}$ 's are i.i.d. \pm 1 random variables and $\delta_{i j}$ is an indicator random variable for the event $\Pi_{i j} \neq 0$; (ii) $\sum_{i=1}^{m} \delta_{i j}=1$ for each $j=1, \ldots, n$; (iii) for any $S \subset[n], \mathbb{E}\left(\Pi_{j \in S} \delta_{i j}\right)=m^{-|S|}$; (iv) the columns of $\Pi$ are i.i.d. Furthermore, there is a universal constant $C_{e}$ such that $\max _{i=1, \ldots, n} \Omega_{e, i i} \leq C_{e}$. Suppose that

$$
\frac{\left(d^{2}+1\right) m}{n}+\frac{\left(d^{2}+d\right)}{m} \leq \frac{\delta_{\Pi} \varepsilon_{\Pi}^{2}}{8 C_{e}^{2}} .
$$

Let

$$
A\left(\Omega_{e}, m, n\right)=\Omega_{e}+\frac{1}{m}\left\{\operatorname{tr}\left(\Omega_{e}\right) I_{n}-\Omega_{e}\right\}
$$

Then, we have that

$$
\mathbb{P}\left(\left\|U^{T} \Pi^{T} \Pi \Omega_{e} \Pi^{T} \Pi U-U^{T} A\left(\Omega_{e}, m, n\right) U\right\|_{2}>\frac{n}{m} \varepsilon_{\Pi}\right) \leq \delta_{\Pi} .
$$


This lemma states that Condition CS is satisfied for countsketch, provided that all the diagonal elements of $\Omega_{e}$ are bounded by a universal constant, $d^{2} m / n=o\left(\delta_{\Pi} \varepsilon_{\Pi}^{2}\right)$ and $d^{2} / m=o\left(\delta_{\Pi} \varepsilon_{\Pi}^{2}\right)$. The rate conditions in (18) are not stringent. When $n$ is very large, $d$ is of a moderate size and $\delta_{\Pi}$ and $\varepsilon_{\Pi}$ are given, there is a range of $m$ that satisfies $(18)$.

Proof of Lemma 5 Since we assume that each diagonal element of $\Omega_{e}$ is bounded by a universal constant $C_{e}$,

$$
\operatorname{tr}\left(\Omega_{e}^{2}\right) \leq C_{e}^{2} n, \operatorname{tr}\left(\Omega_{e}\right)=C_{e} n, \text { and }\left\|\Omega_{e}\right\|_{2}=C_{e}
$$

Then Lemma F.1, which is given in Appendix F, implies that

$$
\begin{aligned}
& \mathbb{P}\left(\left\|U^{T} \Pi^{T} \Pi \Omega_{e} \Pi^{T} \Pi U-U^{T} A\left(\Omega_{e}, m, n\right) U\right\|_{2}>\epsilon\right) \\
& \leq 2 \epsilon^{-2}\left\{\frac{2 d^{2}(m-1)}{m^{2}} \operatorname{tr}\left(\Omega_{e}^{2}\right)+\frac{2 d^{2}}{m^{2}}\left\|\Omega_{e}\right\|_{2} \operatorname{tr}\left(\Omega_{e}\right)+\frac{d^{2}}{m^{3}}\left\{\left[\operatorname{tr}\left(\Omega_{e}\right)\right]^{2}+2\left\|\Omega_{e}\right\|_{2}^{2}\right\}\right. \\
& \left.\quad+\frac{2}{m} \operatorname{tr}\left(\Omega_{e}^{2}\right)+\frac{2}{m^{2}} \operatorname{tr}\left(\Omega_{e}\right)+\frac{1}{m^{3}}\left\{d\left[\operatorname{tr}\left(\Omega_{e}\right)\right]^{2}+2 \operatorname{tr}\left(\Omega_{e}^{2}\right)\right\}\right\} \\
& \leq 2 \epsilon^{-2}\left\{\frac{2 d^{2}(m-1)}{m^{2}} C_{e}^{2} n+\frac{2 d^{2}}{m^{2}} C_{e}^{2} n+\frac{d^{2}}{m^{3}}\left\{C_{e}^{2} n^{2}+2 C_{e}^{2}\right\}\right. \\
& \left.\quad+\frac{2}{m} C_{e}^{2} n+\frac{2}{m^{2}} C_{e} n+\frac{1}{m^{3}}\left\{d C_{e}^{2} n^{2}+2 C_{e}^{2} n\right\}\right\} \\
& \leq 8 C_{e}^{2} \epsilon^{-2}\left(\frac{\left(d^{2}+1\right) n}{m}+\frac{\left(d^{2}+d\right) n^{2}}{m^{3}}\right) .
\end{aligned}
$$

If we take $\epsilon=\frac{n}{m} \varepsilon_{\Pi}$, then

$$
\mathbb{P}\left(\left\|U^{T} \Pi^{T} \Pi \Omega_{e} \Pi^{T} \Pi U-U^{T} A\left(\Omega_{e}, m, n\right) U\right\|_{2}>\frac{n}{m} \varepsilon_{\Pi}\right) \leq 8 C_{e}^{2} \varepsilon_{\Pi}^{-2}\left(\frac{\left(d^{2}+1\right) m}{n}+\frac{\left(d^{2}+d\right)}{m}\right) .
$$

To satisfy the probability above is bounded by $\delta_{\Pi}$, we need to assume that

$$
8 C_{e}^{2} \varepsilon_{\Pi}^{-2}\left(\frac{\left(d^{2}+1\right) m}{n}+\frac{\left(d^{2}+d\right)}{m}\right) \leq \delta_{\Pi},
$$

which is imposed by (18). Q.E.D.

\section{Proof of JL Lemma}

The original proof assumes that $\Pi$ is Gaussian 18 We highlight arguments for this case using properties of sub-exponential random variables. For arbitrary $(i, j)$, define $u=\left(a_{i}-a_{j}\right) \in \mathbb{R}^{n}$ and

\footnotetext{
${ }^{18}$ Subsequent proofs by Dasgupta and Gupta (2003), Indyk and Motwani (1998), Matousek (2008) use different proof techniques to obtain tighter bounds. Fedoruk et al. (2018) summaries the evolution of the Lemma. Others consider non-Gaussian $\Pi$ matrices that are less costly to store.
} 
$v=\Pi a_{i}-\Pi a_{j}=\frac{1}{\sqrt{m}} \Pi u \in \mathbb{R}^{m}$. Note that $v_{i}=\frac{1}{\sqrt{m}} \sum_{j} \Pi_{i j} u_{j} \sim N\left(0, \frac{\|u\|_{2}^{2}}{m}\right)$. The Lemma then says $\left|\frac{\|v\|_{2}^{2}}{\|u\|_{2}^{2}}-1\right| \leq \epsilon$. The proof of this statement proceeds in three steps.

(i) show that the expected value of the Euclidean distance of the random projection is equal to the Euclidean distance of the original subspace:

$$
\begin{aligned}
\mathbb{E}\left[\|v\|_{2}^{2}\right] & =\mathbb{E}\left[\sum_{i=1}^{m} v_{i}^{2}\right]=\sum_{i=1}^{m} E\left[v_{i}^{2}\right] \\
& =\sum_{i=1}^{m} \frac{1}{m} \mathbb{E}\left[\left(\sum_{j} \Pi_{i j} u_{i}\right)^{2}\right]=\sum_{i=1}^{m} \frac{1}{m} \sum_{1 \leq j, k \leq n} u_{j} u_{k} \Pi_{i j} \Pi_{i k} \\
& =\sum_{i=1}^{m} \frac{1}{m} \sum_{1 \leq j, k \leq n} u_{j} u_{k} \delta_{j k}=\sum_{i=1}^{m} \frac{1}{m} \sum_{1 \leq j \leq n} u_{j}^{2} \\
& =\sum_{j \leq n} u_{j}^{2}=\|u\|_{2}^{2} .
\end{aligned}
$$

(ii) show that (i) holds with high probability. For this, let $Z_{i}=\frac{\Pi_{i} u}{\|u\|}$. Then $\sum_{i=1}^{m} Z_{i}^{2}=\frac{m\|v\|_{2}^{2}}{\|u\|_{2}^{2}}$ is $\chi_{m}^{2}$, hence sub-exponential. ${ }^{19}$; A two-sided tail bound yields

$$
P\left(\frac{1}{m}\left|\sum_{k=1}^{m} Z_{k}^{2}-1\right| \geq \epsilon\right)=P\left(\left|\frac{\|v\|_{2}^{2}}{\|u\|_{2}}-1\right|>\epsilon\right) \leq 2 e^{-m \epsilon^{2} / 8} .
$$

(iii) apply union bound: step (ii) holds for all $d^{2}$ pairs of $(i, j)$, so the overall failure probability is at most $2\left(\begin{array}{l}d \\ 2\end{array}\right) e^{-m \epsilon^{2} / 8}$. Given a error rate $\epsilon$, this failure probability can be driven below $\delta$ by making $m \geq \frac{C}{\epsilon^{2}}(\log d / \delta)$ for large enough $C$.

\footnotetext{
${ }^{19} \mathrm{~A}$ random variable $X$ with mean $\mu$ is sub-exponential with parameters $(\nu, b)$ if $\mathbb{E}\left[e^{\lambda(X-\mu)}\right] \leq e^{\nu^{2} \lambda^{2} / 2}$. If $Z_{k} \sim$ $N(0,1), Z_{k}^{2} \sim \chi_{m}^{2}$ is sub-exponential with $(\nu, b)=(2,4)$ and has two-sided tail bound $P\left(\left|\frac{1}{\sqrt{m}} \sum_{k=1}^{m} Z_{k}^{2}-1\right| \geq \epsilon\right) \leq$ $2 e^{-m \epsilon^{2} / 8}, \quad \epsilon \in(0,1)$. See, for example, Wainwright 2019. Chapter 2) or Vershynin (2017, lecture 1).
} 


\section{Streaming Implementation of Countsketch}

\section{CountSketch, Streaming:}

Input: Given $A \in \mathbb{R}^{n \times d}$ and $m<n$

Output: $\widetilde{A} \in \mathbb{R}^{m \times d}$, initialized to zero

1 for $s=1: n$ do

\begin{tabular}{l|l}
$\mathbf{2}$ & sampled $h($ from $[1, \ldots m])$ \\
$\mathbf{3}$ & sample $g$ from $[+1,-1]$ \\
$\mathbf{4}$ & $\widetilde{A}_{(h(s))}:=\widetilde{A}_{(h(s))}+g \times A_{(h(s))}$
\end{tabular}

The example considered in the main text is the same as $C=\Pi A$, where $n=9$,

$$
\Pi=\left(\begin{array}{ccccccccc}
0 & 0 & 1 & 0 & 1 & -1 & 0 & 0 & 1 \\
-1 & 0 & 0 & -1 & 0 & 0 & 0 & -1 & 0 \\
0 & -1 & 0 & 0 & 0 & 0 & 1 & 0 & 0
\end{array}\right) \text { and } A=\left(\begin{array}{c}
A_{3}+A_{5}-A_{6}+A_{9} \\
A_{1}-A_{4}-A_{8} \\
A_{2}+A_{7}
\end{array}\right)
$$

Hence if $d=4$,

$$
\widetilde{A}=\left(\begin{array}{cccc}
A_{31}+A_{51}-A_{61}+A_{91} & A_{32}+A_{52}-A_{62}+A_{92} & A_{33}+A_{53}-A_{63}+A_{93} & A_{34}+A_{54}-A_{64}+A_{94} \\
-A_{11}-A_{41}-A_{81} & -A_{12}-A_{42}-A_{82} & -A_{13}-A_{43}-A_{83} & -A_{14}-A_{44}-A_{84} \\
-A_{21}+A_{71} & -A_{22}+A_{72} & -A_{23}+A_{73} & -A_{24}+A_{74}
\end{array}\right)
$$

Consider now the streaming approach when the random draws of $h$ and $g$ are

$$
\left(\begin{array}{l}
h \\
g
\end{array}\right)=\left(\begin{array}{ccccccccc}
2 & 3 & 1 & 2 & 1 & 1 & 3 & 2 & 1 \\
-1 & -1 & +1 & -1 & +1 & -1 & +1 & -1 & 1
\end{array}\right)
$$

1. $s=1: A_{(1)}=\left(\begin{array}{llll}A_{11} & A_{12} & A_{13} & A_{14}\end{array}\right), h=2, g=-1$. Updating $A_{h(1)}=A_{2}$ gives

$$
\widetilde{A}=\left(\begin{array}{cccc}
0 & 0 & 0 & 0 \\
A_{11} \times-1 & A_{12} \times-1 & A_{13} \times-1 & A_{14}-1 \\
0 & 0 & 0 & 0
\end{array}\right) .
$$

2. $s=2: A_{(2)}=\left(\begin{array}{llll}A_{21} & A_{22} & A_{23} & A_{24}\end{array}\right), h=3, g=-1$. Updating $A_{h(2)}=A_{3}$ gives

$$
\widetilde{A}=\left(\begin{array}{cccc}
0 & 0 & 0 & 0 \\
-A_{11} & -A_{12} & -A_{13} & -A_{14} \\
A_{21} \times-1 & A_{22} \times-1 & A_{23} \times-1 & A_{24} \times-1
\end{array}\right)
$$

3. $s=3: A_{(3)}=\left(\begin{array}{llll}A_{31} & A_{32} & A_{33} & A_{34}\end{array}\right), h=1, g=1$. Updating $A_{h(3)}=A_{1}$ gives

$$
\widetilde{A}=\left(\begin{array}{cccc}
A_{31} & A_{32} & A_{33} & A_{34} \\
-A_{11} & -A_{12} & -A_{13} & -A_{14} \\
-A_{21} & -A_{22} & -A_{23} & -A_{24}
\end{array}\right)
$$

4. $s=4: A_{(4)}=\left(\begin{array}{llll}A_{41} & A_{42} & A_{43} & A_{44}\end{array}\right), h=2, g=-1$. Updating $A_{h(4)}=A_{2}$ gives

$$
\widetilde{A}=\left(\begin{array}{cccc}
A_{31} \times 1 & A_{32} \times 1 & A_{33} \times 1 & A_{34} \times 1 \\
-A_{11}+\left(A_{41} \times-1\right) & -A_{12}+\left(A_{42} \times-1\right) & -A_{13}+\left(A_{43} \times-1\right) & -A_{14}+\left(A_{44} \times-1\right) \\
-A_{21} & -A_{22} & -A_{23} & -A_{24}
\end{array}\right)
$$


5. $s=5: A_{(5)}=\left(\begin{array}{llll}A_{51} & A_{52} & A_{53} & A_{54}\end{array}\right), h=1, g=1$. Updating $A_{h(5)}=A_{1}$ gives

$$
\widetilde{A}=\left(\begin{array}{cccc}
A_{31}+A_{51} & A_{32}+A_{52} & A_{33}+A_{53} & A_{34}+A_{54} \\
-A_{11}-A_{41} & -A_{12}-A_{42} & -A_{13}-A_{43} & -A_{14}-A_{44} \\
A_{21} \times-1 & A_{22} \times-1 & A_{23} \times-1 & A_{24} \times-1
\end{array}\right)
$$

6. $s=6: A_{(6)}=\left(\begin{array}{llll}A_{61} & A_{62} & A_{63} & A_{64}\end{array}\right), h=1, g=-1$. Updating $A_{h(6)}=A_{1}$ gives

$$
\widetilde{A}=\left(\begin{array}{cccc}
A_{31}+A_{51}-A_{61} & A_{32}+A_{52}-A_{62} & A_{33}+A_{53}-A_{63} & A_{34}+A_{54}-A_{64} \\
-A_{11}-A_{41} & -A_{12}-A_{42} & -A_{13}-A_{43} & -A_{14}-A_{44} \\
A_{21} \times-1 & A_{22} \times-1 & A_{23} \times-1 & A_{24} \times-1
\end{array}\right)
$$

7. $s=7: A_{(7)}=\left(\begin{array}{llll}A_{71} & A_{72} & A_{73} & A_{74}\end{array}\right), h=3, g=1$. Updating $A_{h(7)}=A_{3}$ gives

$$
\widetilde{A}=\left(\begin{array}{cccc}
A_{31}+A_{51}-A_{61} & A_{32}+A_{52}-A_{62} & A_{33}+A_{53}-A_{63} & A_{34}+A_{54}-A_{64} \\
-A_{11}-A_{41} & -A_{12}-A_{42} & -A_{13}-A_{43} & -A_{14}-A_{44} \\
-A_{21}+A_{71} & -A_{22}+A_{72} & -A_{23}+A_{73} & -A_{24}+A_{74}
\end{array}\right)
$$

8. $s=8: A_{(8)}=\left(\begin{array}{llll}A_{81} & A_{82} & A_{83} & A_{84}\end{array}\right), h=2, g=-1$. Updating $A_{h(8)}=A_{2}$ gives

$$
\widetilde{A}=\left(\begin{array}{cccc}
A_{31}+A_{51}-A_{61} & A_{32}+A_{52}-A_{62} & A_{33}+A_{53}-A_{63} & A_{34}+A_{54}-A_{64} \\
-A_{11}-A_{41}-A_{81} & -A_{12}-A_{42}-A_{82} & -A_{13}-A_{43}-A_{83} & -A_{14}-A_{44}-A_{84} \\
-A_{21}+A_{71} & -A_{22}+A_{72} & -A_{23}+A_{73} & -A_{24}+A_{74}
\end{array}\right)
$$

9. $s=9: A_{(9)}=\left(\begin{array}{llll}A_{91} & A_{92} & A_{93} & A_{94}\end{array}\right), h=1, g=1$. Updating $A_{h(9)}=A_{1}$ gives

$$
\widetilde{A}=\left(\begin{array}{cccc}
A_{31}+A_{51}-A_{61}+A_{91} & A_{32}+A_{52}-A_{62}+A_{92} & A_{33}+A_{53}-A_{63}+A_{93} & A_{34}+A_{54}-A_{64}+A_{94} \\
-A_{11}-A_{41}-A_{81} & -A_{12}-A_{42}-A_{82} & -A_{13}-A_{43}-A_{83} & -A_{14}-A_{44}-A_{84} \\
-A_{21}+A_{71} & -A_{22}+A_{72} & -A_{23}+A_{73} & -A_{24}+A_{74}
\end{array}\right) .
$$

This is precisely $\Pi A$. 


\section{E General Version of Theorem 1}

In this section, we give a general version of Theorem 1 under heteroskedasticity.

Theorem 4 Let $c$ be a known $K \times 1$ vector. Assume that $e_{i} \sim\left(0, \Omega_{e, i i}\right)$, where $\Omega_{e, i i}$ denote the ith diagonal element of $\Omega_{e}$. Under OLS and PI3, it holds with probability at least $1-\delta_{\sigma}$ that

$$
\frac{\operatorname{MSE}\left(x_{0}^{T} \widetilde{\beta} \mid \Pi\right)}{\operatorname{MSE}\left(x_{0}^{T} \hat{\beta}\right)} \leq\left(\frac{\max _{i} \Omega_{e, i i}}{\min _{i} \Omega_{e, i i}}\right)\left(\frac{n}{m}\right) \frac{\left(1+\varepsilon_{\sigma}\right)}{\left(1-\varepsilon_{\sigma}\right)^{2}} \text {. }
$$

Proof of Theorem 4 We have $\mathbb{V}(\widetilde{\beta} \mid \Pi)=\left(\widetilde{X}^{T} \widetilde{X}\right)^{-1} \widetilde{X}^{T} \Pi \Omega_{e} \Pi^{T} \widetilde{X}\left(\widetilde{X}^{T} \widetilde{X}\right)^{-1}$. The quantity of interest is

$$
\begin{aligned}
& c^{T} \mathbb{V}(\widetilde{\beta} \mid \Pi) c-\frac{n}{m} c^{T} \mathbb{V}(\hat{\beta}) c \\
& =c^{T} A_{1} B_{1} A_{1} c-c^{T} A_{2} B_{2} A_{2} c \\
& =c^{T}\left(A_{1}-A_{2}\right) B_{1}\left(A_{1}-A_{2}\right) c+2 c^{T} A_{2} B_{1}\left(A_{1}-A_{2}\right) c+c^{T} A_{2}\left(B_{1}-B_{2}\right) A_{2} c,
\end{aligned}
$$

where $A_{1}=\left(X^{T} \Pi^{T} \Pi X\right)^{-1}, B_{1}=X^{T} \Pi^{T} \Pi \Omega_{e} \Pi^{T} \Pi X, A_{2}=\left(X^{T} X\right)^{-1}$, and $B_{2}=\frac{n}{m}\left(X^{T} \Omega_{e} X\right)$. Write

$$
\begin{aligned}
& \left|c^{T} \mathbb{V}(\widetilde{\beta} \mid \Pi) c-\frac{n}{m} c^{T} \mathbb{V}(\hat{\beta}) c\right| \\
& \leq\left|c^{T}\left(A_{1}-A_{2}\right) B_{2}\left(A_{1}-A_{2}\right) c\right|+\left|c^{T}\left(A_{1}-A_{2}\right)\left(B_{1}-B_{2}\right)\left(A_{1}-A_{2}\right) c\right| \\
& +2\left|c^{T} A_{2} B_{2}\left(A_{1}-A_{2}\right) c\right|+2\left|c^{T} A_{2}\left(B_{1}-B_{2}\right)\left(A_{1}-A_{2}\right) c\right|+\left|c^{T} A_{2}\left(B_{1}-B_{2}\right) A_{2} c\right| .
\end{aligned}
$$

Let

$$
C\left(\Omega_{e}\right)=\frac{\max _{i} \Omega_{e, i i}}{\min _{i} \Omega_{e, i i}}
$$

We will bound each of the terms above by establishing the following lemma.

Lemma 6 The following statements hold with probability at least $1-\delta_{\sigma}$ :

$$
\begin{aligned}
\left|\frac{c^{T} A_{2} B_{2}\left(A_{1}-A_{2}\right) c}{c^{T} \mathbb{V}(\hat{\beta}) c}\right| & \leq \frac{n}{m} C\left(\Omega_{e}\right)^{1 / 2} \frac{\varepsilon_{\sigma}}{\left(1-\varepsilon_{\sigma}\right)}, \\
\left|\frac{c^{T}\left(A_{1}-A_{2}\right) B_{2}\left(A_{1}-A_{2}\right) c \mid}{c^{T} \mathbb{V}(\hat{\beta}) c}\right| & \leq \frac{n}{m} C\left(\Omega_{e}\right) \frac{\varepsilon_{\sigma}^{2}}{\left(1-\varepsilon_{\sigma}\right)^{2}}, \\
\left|\frac{c^{T}\left(A_{1}-A_{2}\right)\left(B_{1}-B_{2}\right)\left(A_{1}-A_{2}\right) c}{c^{T} \mathbb{V}(\hat{\beta}) c}\right| & \leq \frac{n}{m} C\left(\Omega_{e}\right) \frac{\varepsilon_{\sigma}^{2}}{\left(1-\varepsilon_{\sigma}\right)^{2}} \varepsilon_{\sigma}, \\
\left|\frac{c^{T} A_{2}\left(B_{1}-B_{2}\right)\left(A_{1}-A_{2}\right) c}{c^{T} \mathbb{V}(\hat{\beta}) c}\right| & \leq \frac{n}{m} C\left(\Omega_{e}\right)^{1 / 2} \frac{\varepsilon_{\sigma}}{\left(1-\varepsilon_{\sigma}\right)} \varepsilon_{\sigma}, \\
\left|\frac{c^{T} A_{2}\left(B_{1}-B_{2}\right) A_{2} c}{c^{T} \mathbb{V}(\hat{\beta}) c}\right| & \leq \frac{n}{m} \varepsilon_{\sigma} .
\end{aligned}
$$


Proof of Lemma 6 To show part 20a), define $W=\left(U^{T} \Omega_{e} U\right)^{1 / 2} \Sigma^{-1} V^{T}$. Note that

$$
\begin{aligned}
\mathbb{V}(\hat{\beta}) & =\left(X^{T} X\right)^{-1}\left(X^{T} \Omega_{e} X\right)\left(X^{T} X\right)^{-1} \\
& =V \Sigma^{-1} U^{T} \Omega_{e} U \Sigma^{-1} V^{T} \\
& =W^{T} W .
\end{aligned}
$$

Write

$$
\begin{aligned}
A_{2} B_{2}\left(A_{1}-A_{2}\right) & =\frac{n}{m}\left(X^{T} X\right)^{-1}\left(X^{T} \Omega_{e} X\right)\left[\left(X^{T} \Pi^{T} \Pi X\right)^{-1}-\left(X^{T} X\right)^{-1}\right] \\
& =\frac{n}{m} V \Sigma^{-1} U^{T} \Omega_{e} U\left[\left(U^{T} \Pi^{T} \Pi U\right)^{-1}-I_{n}\right] \Sigma^{-1} V^{T} \\
& =\frac{n}{m} V \Sigma^{-1} U^{T} \Omega_{e} U\left[\left(U^{T} \Pi^{T} \Pi U\right)^{-1}-I_{n}\right]\left(U^{T} \Omega_{e} U\right)^{-1 / 2}\left(U^{T} \Omega_{e} U\right)^{1 / 2} \Sigma^{-1} V^{T} \\
& =\frac{n}{m} W^{T}\left(U^{T} \Omega_{e} U\right)^{1 / 2}\left[\left(U^{T} \Pi^{T} \Pi U\right)^{-1}-I_{n}\right]\left(U^{T} \Omega_{e} U\right)^{-1 / 2} W .
\end{aligned}
$$

Then

$$
\begin{aligned}
\left|\frac{c^{T} A_{2} B_{2}\left(A_{1}-A_{2}\right) c}{c^{T} \mathbb{V}(\hat{\beta}) c}\right| & =\frac{n}{m}\left|\frac{c^{T} W^{T}\left(U^{T} \Omega_{e} U\right)^{1 / 2}\left[\left(U^{T} \Pi^{T} \Pi U\right)^{-1}-I_{n}\right]\left(U^{T} \Omega_{e} U\right)^{-1 / 2} W c}{c^{T} W^{T} W c}\right| \\
& \leq \frac{n}{m}\left\|\left(U^{T} \Omega_{e} U\right)^{1 / 2}\right\|_{2}\left\|\left(U^{T} \Pi^{T} \Pi U\right)^{-1}-I_{n}\right\|_{2}\left\|\left(U^{T} \Omega_{e} U\right)^{-1 / 2}\right\|_{2} \\
& \leq \frac{n}{m} C\left(\Omega_{e}\right)^{1 / 2} \frac{\varepsilon_{\sigma}}{\left(1-\varepsilon_{\sigma}\right)},
\end{aligned}
$$

which proves part 20a).

For the second part, write

$$
\begin{aligned}
& \left(A_{1}-A_{2}\right) B_{2}\left(A_{1}-A_{2}\right) \\
& =\frac{n}{m}\left[\left(X^{T} \Pi^{T} \Pi X\right)^{-1}-\left(X^{T} X\right)^{-1}\right]\left(X^{T} \Omega_{e} X\right)\left[\left(X^{T} \Pi^{T} \Pi X\right)^{-1}-\left(X^{T} X\right)^{-1}\right] \\
& =\frac{n}{m} V \Sigma^{-1}\left[\left(U^{T} \Pi^{T} \Pi U\right)^{-1}-I_{n}\right] U^{T} \Omega_{e} U\left[\left(U^{T} \Pi^{T} \Pi U\right)^{-1}-I_{n}\right] \Sigma^{-1} V^{T} \\
& =\frac{n}{m} W^{T}\left(U^{T} \Omega_{e} U\right)^{-1 / 2}\left[\left(U^{T} \Pi^{T} \Pi U\right)^{-1}-I_{n}\right] U^{T} \Omega_{e} U\left[\left(U^{T} \Pi^{T} \Pi U\right)^{-1}-I_{n}\right]\left(U^{T} \Omega_{e} U\right)^{-1 / 2} W .
\end{aligned}
$$

Thus,

$$
\left|\frac{c^{T}\left(A_{1}-A_{2}\right) B_{2}\left(A_{1}-A_{2}\right) c}{c^{T} \mathbb{V}(\hat{\beta}) c}\right| \leq \frac{n}{m} C\left(\Omega_{e}\right) \frac{\varepsilon_{\sigma}^{2}}{\left(1-\varepsilon_{\sigma}\right)^{2}},
$$

which proves part 20b. 
For the third part, write

$$
\begin{aligned}
& \left(A_{1}-A_{2}\right)\left(B_{1}-B_{2}\right)\left(A_{1}-A_{2}\right) \\
& =V \Sigma^{-1}\left[\left(U^{T} \Pi^{T} \Pi U\right)^{-1}-I_{n}\right]\left[U^{T} \Omega_{e}^{1 / 2} \Pi^{T} \Pi \Pi^{T} \Pi \Omega_{e}^{1 / 2} U-\frac{n}{m} U^{T} \Omega_{e}^{1 / 2} \Omega_{e}^{1 / 2} U\right]\left[\left(U^{T} \Pi^{T} \Pi U\right)^{-1}-I_{n}\right] \Sigma^{-1} V^{T} \\
& =W^{T}\left(U^{T} \Omega_{e} U\right)^{-1 / 2}\left[\left(U^{T} \Pi^{T} \Pi U\right)^{-1}-I_{n}\right]\left(U^{T} \Omega_{e} U\right)^{1 / 2} \\
& \times\left(U^{T} \Omega_{e} U\right)^{-1 / 2}\left[U^{T} \Omega_{e}^{1 / 2} \Pi^{T} \Pi \Pi^{T} \Pi \Omega_{e}^{1 / 2} U-\frac{n}{m} U^{T} \Omega_{e}^{1 / 2} \Omega_{e}^{1 / 2} U\right]\left(U^{T} \Omega_{e} U\right)^{-1 / 2} \\
& \times\left(U^{T} \Omega_{e} U\right)^{1 / 2}\left[\left(U^{T} \Pi^{T} \Pi U\right)^{-1}-I_{n}\right]\left(U^{T} \Omega_{e} U\right)^{-1 / 2} W .
\end{aligned}
$$

Define $U_{e}=\Omega_{e}^{1 / 2} U\left(U^{T} \Omega_{e} U\right)^{-1 / 2}$, so that

$$
\begin{aligned}
& \left(U^{T} \Omega_{e} U\right)^{-1 / 2}\left[U^{T} \Omega_{e}^{1 / 2} \Pi^{T} \Pi \Pi^{T} \Pi \Omega_{e}^{1 / 2} U-\frac{n}{m} U^{T} \Omega_{e}^{1 / 2} \Omega_{e}^{1 / 2} U\right]\left(U^{T} \Omega_{e} U\right)^{-1 / 2} \\
& =U_{e}^{T} \Pi^{T} \Pi \Pi^{T} \Pi U_{e}-\frac{n}{m} U_{e}^{T} U_{e} .
\end{aligned}
$$

Now observe that

$$
\begin{aligned}
& \left\|U_{e}^{T} \Pi^{T} \Pi \Pi^{T} \Pi U_{e}-\frac{n}{m} U_{e}^{T} U_{e}\right\|_{2} \\
& \leq\left\|U_{e}^{T} \Pi^{T} \Pi \Pi^{T} \Pi U_{e}-\frac{n}{m} U_{e}^{T} \Pi^{T} \Pi U_{e}\right\|_{2}+\frac{n}{m}\left\|U_{e}^{T} \Pi^{T} \Pi U_{e}-U_{e}^{T} U_{e}\right\|_{2} \\
& \leq \frac{n}{m} \varepsilon_{\sigma} .
\end{aligned}
$$

Thus,

$$
\left|\frac{c^{T}\left(A_{1}-A_{2}\right)\left(B_{1}-B_{2}\right)\left(A_{1}-A_{2}\right) c}{c^{T} \mathbb{V}(\hat{\beta}) c}\right| \leq C\left(\Omega_{e}\right) \frac{\varepsilon_{\sigma}^{2}}{\left(1-\varepsilon_{\sigma}\right)^{2}} \frac{n}{m} \varepsilon_{\sigma},
$$

which proves part 20c).

Similarly,

$$
\begin{aligned}
& A_{2}\left(B_{1}-B_{2}\right)\left(A_{1}-A_{2}\right) \\
& =V \Sigma^{-1}\left[U^{T} \Omega_{e}^{1 / 2} \Pi^{T} \Pi \Pi^{T} \Pi \Omega_{e}^{1 / 2} U-\frac{n}{m} U^{T} \Omega_{e}^{1 / 2} \Omega_{e}^{1 / 2} U\right]\left[\left(U^{T} \Pi^{T} \Pi U\right)^{-1}-I_{n}\right] \Sigma^{-1} V^{T} .
\end{aligned}
$$

Then

$$
\left|\frac{c^{T} A_{2}\left(B_{1}-B_{2}\right)\left(A_{1}-A_{2}\right) c}{c^{T} \mathbb{V}(\hat{\beta}) c}\right| \leq C\left(\Omega_{e}\right)^{1 / 2} \frac{\varepsilon_{\sigma}}{\left(1-\varepsilon_{\sigma}\right)} \frac{n}{m} \varepsilon_{\sigma},
$$

which proves part 20d.

Finally,

$$
A_{2}\left(B_{1}-B_{2}\right) A_{2}=V \Sigma^{-1}\left[U^{T} \Omega_{e}^{1 / 2} \Pi^{T} \Pi \Pi^{T} \Pi \Omega_{e}^{1 / 2} U-\frac{n}{m} U^{T} \Omega_{e}^{1 / 2} \Omega_{e}^{1 / 2} U\right] \Sigma^{-1} V^{T} .
$$


Therefore,

$$
\left|\frac{c^{T} A_{2}\left(B_{1}-B_{2}\right) A_{2} c}{c^{T} \mathbb{V}(\hat{\beta}) c}\right| \leq \frac{n}{m} \varepsilon_{\sigma},
$$

which proves part (20). Q.E.D.

We now return to the proof of the theorem. Applying 20a-20e and simplifying,

$$
\begin{aligned}
\left|\frac{c^{T} \mathbb{V}(\widetilde{\beta} \mid \Pi) c-\frac{n}{m} c^{T} \mathbb{V}(\hat{\beta}) c}{c^{T} \mathbb{V}(\hat{\beta}) c}\right| & \leq C\left(\Omega_{e}\right) \frac{n}{m}\left[\frac{\varepsilon_{\sigma}\left(2-\varepsilon_{\sigma}\right)}{\left(1-\varepsilon_{\sigma}\right)^{2}}\left(1+\varepsilon_{\sigma}\right)+\varepsilon_{\sigma}\right] \\
\left|\frac{c^{T} \mathbb{V}(\widetilde{\beta} \mid \Pi) c-c^{T} \mathbb{V}(\hat{\beta}) c}{c^{T} \mathbb{V}(\hat{\beta}) c}\right| & \left.\leq C\left(\Omega_{e}\right) \frac{n}{m}\left[\frac{\varepsilon_{\sigma}\left(2-\varepsilon_{\sigma}\right)}{\left(1-\varepsilon_{\sigma}\right)^{2}}\left(1+\varepsilon_{\sigma}\right)+\varepsilon_{\sigma}\right)\right]+\frac{n-m}{m}
\end{aligned}
$$

with probability at least $1-\delta_{\sigma}-\delta_{\Pi}$. This in turn implies that with the same probability,

$$
\frac{c^{T} \mathbb{V}(\widetilde{\beta} \mid \Pi) c}{c^{T} \mathbb{V}(\hat{\beta}) c} \leq C\left(\Omega_{e}\right) \frac{n}{m} \frac{\left(1+\varepsilon_{\sigma}\right)}{\left(1-\varepsilon_{\sigma}\right)^{2}} .
$$

Q.E.D. 


\section{F $\quad$ Subspace embedding for $\Pi^{T} \Pi \Psi \Pi^{T} \Pi$}

In this appendix, we consider $\Pi^{T} \Pi \Psi \Pi^{T} \Pi$ for a diagonal matrix $\Psi$ in the case of countsketch.

Lemma 7 Let $\Pi \in \mathbb{R}^{n \times d}$ be a random matrix such that (i) the $(i, j)$ element $\Pi_{i j}$ of $\Pi$ is $\Pi_{i j}=\delta_{i j} \sigma_{i j}$, where $\sigma_{i j}$ 's are i.i.d. \pm 1 random variables and $\delta_{i j}$ is an indicator random variable for the event $\Pi_{i j} \neq 0$; (ii) $\sum_{i=1}^{m} \delta_{i j}=1$ for each $j=1, \ldots, n$; (iii) for any $S \subset[n], \mathbb{E}\left(\Pi_{j \in S} \delta_{i j}\right)=m^{-|S|}$; (iv) the columns of $\Pi$ are i.i.d. Choose

$$
A(\Psi, m, n)=\Psi+\frac{1}{m}\left\{\operatorname{tr}(\Psi) I_{n}-\Psi\right\} .
$$

Then

$$
\begin{aligned}
& \mathbb{P}\left(\left\|U^{T} \Pi^{T} \Pi \Psi \Pi^{T} \Pi U-U^{T} A(\Psi, m, n) U\right\|_{2}>\epsilon\right) \\
& \leq 2 \epsilon^{-2}\left\{\frac{2 d^{2}(m-1)}{m^{2}} \operatorname{tr}\left(\Psi^{2}\right)+\frac{2 d^{2}}{m^{2}}\|\Psi\|_{2} \operatorname{tr}(\Psi)+\frac{d^{2}}{m^{3}}\left\{[\operatorname{tr}(\Psi)]^{2}+2\|\Psi\|_{2}^{2}\right\}\right. \\
& \left.\quad+\frac{2}{m} \operatorname{tr}\left(\Psi^{2}\right)+\frac{2}{m^{2}} \operatorname{tr}(\Psi)+\frac{1}{m^{3}}\left\{d[\operatorname{tr}(\Psi)]^{2}+2 \operatorname{tr}\left(\Psi^{2}\right)\right\}\right\} .
\end{aligned}
$$

Proof of Lemma 7 To show the desired result, we follow Nelson and Nguyen (2013). That is, we start with the following moment inequality:

$$
\begin{aligned}
& \mathbb{P}\left(\left\|U^{T} \Pi^{T} \Pi \Psi \Pi^{T} \Pi U-U^{T} A(\Psi, m, n) U\right\|_{2}>\epsilon\right) \\
& \leq \epsilon^{-2} \mathbb{E}\left[\left\|U^{T} \Pi^{T} \Pi \Psi \Pi^{T} \Pi U-U^{T} A(\Psi, m, n) U\right\|_{F}^{2}\right] .
\end{aligned}
$$

For countsketch, we have one non-zero entry for each column. This implies that $\Pi \Psi \Pi^{T}$ is a diagonal matrix. Hence,

$$
\left[\Pi \Psi \Pi^{T}\right]_{i i}=\sum_{k=1}^{n} \Psi_{k k} \Pi_{i k}^{2}=\sum_{k=1}^{n} \Psi_{k k} \delta_{i k}
$$

Now

$$
\left[\Pi^{T} \Pi \Psi \Pi^{T} \Pi\right]_{\ell \ell^{\prime}}=\sum_{i=1}^{m} \sum_{k=1}^{n} \Pi_{i \ell} \Psi_{k k} \delta_{i k} \Pi_{i \ell^{\prime}}=\sum_{i=1}^{m} \sum_{k=1}^{n} \Psi_{k k} \delta_{i k} \delta_{i \ell} \delta_{i \ell^{\prime}} \sigma_{i \ell} \sigma_{i \ell^{\prime}},
$$

where the last equality comes from the fact that $\Pi_{i j}=\delta_{i j} \sigma_{i j}$. Then

$$
\begin{aligned}
{\left[U^{T} \Pi^{T} \Pi \Psi \Pi^{T} \Pi U\right]_{u v} } & =\sum_{\ell=1}^{n} \sum_{\ell^{\prime}=1}^{n}\left[\Pi^{T} \Pi \Psi \Pi^{T} \Pi\right]_{\ell \ell^{\prime}} U_{\ell u} U_{\ell^{\prime} v} \\
& =\sum_{\ell=1}^{n} \sum_{\ell^{\prime}=1}^{n} \sum_{i=1}^{m} \sum_{k=1}^{n} \Psi_{k k} \delta_{i k} \delta_{i \ell} \delta_{i \ell^{\prime}} \sigma_{i \ell} \sigma_{i \ell^{\prime}} U_{\ell u} U_{\ell^{\prime} v}
\end{aligned}
$$


Write

$$
\begin{aligned}
{\left[U^{T} \Pi^{T} \Pi \Psi \Pi^{T} \Pi U\right]_{u v} } & =\sum_{\ell=1}^{n} \sum_{i=1}^{m} \sum_{k=1}^{n} \Psi_{k k} \delta_{i k} \delta_{i \ell} U_{\ell u} U_{\ell v} \\
& +\sum_{\ell=1}^{n} \sum_{\ell^{\prime}=1, \ell^{\prime} \neq \ell}^{n} \sum_{i=1}^{m} \sum_{k=1}^{n} \Psi_{k k} \delta_{i k} \delta_{i \ell} \delta_{i \ell^{\prime}} \sigma_{i \ell} \sigma_{i \ell^{\prime}} U_{\ell u} U_{\ell^{\prime} v} \\
& =\sum_{\ell=1}^{n} \sum_{i=1}^{m} \Psi_{\ell \ell} \delta_{i \ell} U_{\ell u} U_{\ell v} \\
& +\sum_{\ell=1}^{n} \sum_{i=1}^{m} \sum_{k=1, k \neq \ell}^{n} \Psi_{k k} \delta_{i k} \delta_{i \ell} U_{\ell u} U_{\ell v} \\
& +\sum_{\ell=1}^{n} \sum_{\ell^{\prime}=1, \ell^{\prime} \neq \ell}^{n} \sum_{i=1}^{m} \sum_{k=1}^{n} \Psi_{k k} \delta_{i k} \delta_{i \ell} \delta_{i \ell^{\prime}} \sigma_{i \ell} \sigma_{i \ell^{\prime}} U_{\ell u} U_{\ell^{\prime} v} \\
& =\sum_{\ell=1}^{n} \Psi_{\ell \ell} U_{\ell u} U_{\ell v} \\
& +\sum_{\ell=1}^{n} \sum_{i=1}^{m} \sum_{k=1, k \neq \ell}^{n} \Psi_{k k} \delta_{i k} \delta_{i \ell} U_{\ell u} U_{\ell v} \\
& +\sum_{\ell=1}^{n} \sum_{\ell^{\prime}=1, \ell^{\prime} \neq \ell}^{n} \sum_{i=1}^{m} \sum_{k=1}^{n} \Psi_{k k} \delta_{i k} \delta_{i \ell} \delta_{i \ell^{\prime}} \sigma_{i \ell} \sigma_{i \ell^{\prime}} U_{\ell u} U_{\ell^{\prime} v}
\end{aligned}
$$

since $\sum_{i=1}^{m} \delta_{i \ell}=1$ for each $\ell$. Recall that we have assumed that for any $S \subset[n], \mathbb{E}\left(\Pi_{j \in S} \delta_{i j}\right)=$ $m^{-|S|}$. Then, note that

$$
\begin{aligned}
& \mathbb{E}\left(\sum_{\ell=1}^{n} \sum_{i=1}^{m} \sum_{k=1, k \neq \ell}^{n} \Psi_{k k} \delta_{i k} \delta_{i \ell} U_{\ell u} U_{\ell v}\right) \\
& =\sum_{\ell=1}^{n} \sum_{i=1}^{m} \sum_{k=1, k \neq \ell}^{n} \Psi_{k k} \mathbb{E}\left(\delta_{i k} \delta_{i \ell}\right) U_{\ell u} U_{\ell v} \\
& =\frac{1}{m} \sum_{\ell=1}^{n} \sum_{k=1, k \neq \ell}^{n} \Psi_{k k} U_{\ell u} U_{\ell v} \\
& =\frac{1}{m} \sum_{\ell=1}^{n}\left[\sum_{k=1}^{n} \Psi_{k k}-\Psi_{\ell \ell}\right] U_{\ell u} U_{\ell v} \\
& =\frac{\operatorname{tr}(\Psi)}{m} 1(u=v)-m^{-1} \sum_{\ell=1}^{n} \Psi_{\ell \ell} U_{\ell u} U_{\ell v} .
\end{aligned}
$$

Since

$$
\sum_{k=1}^{n} \Psi_{k k} U_{k u} U_{k v}=\left[U^{T} \Psi U\right]_{u v}
$$


$U^{T} \Pi^{T} \Pi \Psi \Pi^{T} \Pi U$ should center on

$$
\frac{\operatorname{tr}(\Psi)}{m} U^{T} U+\left(1-\frac{1}{m}\right) U^{T} \Psi U=U^{T}\left[\Psi+\frac{1}{m}\left\{\operatorname{tr}(\Psi) I_{n}-\Psi\right\}\right] U .
$$

If $\Psi=\sigma_{e}^{2} I_{n}$, then its form is

$$
\sigma_{e}^{2} \frac{n}{m} U^{T} U+\sigma_{e}^{2}\left(1-\frac{1}{m}\right) U^{T} U=\sigma_{e}^{2}\left[\frac{n+m-1}{m}\right] U^{T} U .
$$

Now

$$
\begin{aligned}
& {\left[U^{T} \Pi^{T} \Pi \Psi \Pi^{T} \Pi U-U^{T}\left(\Psi+\frac{1}{m}\left\{\operatorname{tr}(\Psi) I_{n}-\Psi\right\}\right) U\right]_{u v}} \\
& =\sum_{\ell=1}^{n} \sum_{k=1, k \neq \ell}^{n} \Psi_{k k}\left[\sum_{i=1}^{m} \delta_{i k} \delta_{i \ell}-\frac{1}{m}\right] U_{\ell u} U_{\ell v} \\
& +\sum_{\ell=1}^{n} \sum_{\ell^{\prime}=1, \ell^{\prime} \neq \ell}^{n} \sum_{k=1}^{n} \Psi_{k k}\left[\sum_{i=1}^{m} \delta_{i k} \delta_{i \ell} \delta_{i \ell^{\prime}} \sigma_{i \ell} \sigma_{i \ell^{\prime}}\right] U_{\ell u} U_{\ell^{\prime} v} \\
& =: T_{1, u v}+T_{2, u v} .
\end{aligned}
$$

First consider $T_{1, u v}$. Define

$$
k_{m}(k, \ell):=\sum_{i=1}^{m} \delta_{i k} \delta_{i \ell}-\frac{1}{m}
$$

Write

$$
\begin{aligned}
& k_{m}(k, \ell) k_{m}\left(k^{\prime}, \ell^{\prime}\right) \\
& =\sum_{i=1}^{m} \delta_{i k} \delta_{i \ell} \delta_{i k^{\prime}} \delta_{i \ell^{\prime}}+\sum_{i=1}^{m} \sum_{i^{\prime}=1, i^{\prime} \neq i}^{m} \delta_{i k} \delta_{i \ell} \delta_{i^{\prime} k^{\prime}} \delta_{i^{\prime} \ell^{\prime}}-\frac{1}{m} \sum_{i=1}^{m} \delta_{i k} \delta_{i \ell}-\frac{1}{m} \sum_{i^{\prime}=1}^{m} \delta_{i^{\prime} k^{\prime}} \delta_{i^{\prime} \ell^{\prime}}+\frac{1}{m^{2}} .
\end{aligned}
$$

Then for any $\ell \neq k$ and $\ell^{\prime} \neq k^{\prime}$,

$$
\sum_{i=1}^{m} \delta_{i k} \delta_{i \ell} \delta_{i k^{\prime}} \delta_{i \ell^{\prime}}= \begin{cases}\sum_{i=1}^{m} \delta_{i k} \delta_{i \ell} & \text { if } \ell=\ell^{\prime}, k=k^{\prime} \\ \sum_{i=1}^{m} \delta_{i k} \delta_{i \ell} \delta_{i k^{\prime}} & \text { if } \ell=\ell^{\prime}, k \neq k^{\prime} \\ \sum_{i=1}^{m} \delta_{i k} \delta_{i \ell} \delta_{i \ell^{\prime}} & \text { if } \ell \neq \ell^{\prime}, k=k^{\prime} \\ \sum_{i=1}^{m} \delta_{i k} \delta_{i \ell} & \text { if } \ell \neq \ell^{\prime}, k \neq k^{\prime}, k=\ell^{\prime}, \ell=k^{\prime} \\ \sum_{i=1}^{m} \delta_{i k} \delta_{i \ell} \delta_{i k^{\prime}} & \text { if } \ell \neq \ell^{\prime}, k \neq k^{\prime}, k=\ell^{\prime}, \ell \neq k^{\prime} \\ \sum_{i=1}^{m} \delta_{i k} \delta_{i \ell} \delta_{i \ell^{\prime}} & \text { if } \ell \neq \ell^{\prime}, k \neq k^{\prime}, k \neq \ell^{\prime}, \ell=k^{\prime} \\ \sum_{i=1}^{m} \delta_{i k} \delta_{i \ell} \delta_{i k^{\prime}} \delta_{i \ell^{\prime}} & \text { if } \ell \neq \ell^{\prime}, k \neq k^{\prime}, k \neq \ell^{\prime}, \ell \neq k^{\prime} .\end{cases}
$$


Then

$$
\mathbb{E}\left(\sum_{i=1}^{m} \delta_{i k} \delta_{i \ell} \delta_{i k^{\prime}} \delta_{i \ell^{\prime}}\right)= \begin{cases}m^{-1} & \text { if } \ell=\ell^{\prime}, k=k^{\prime} \\ m^{-2} & \text { if } \ell=\ell^{\prime}, k \neq k^{\prime} \\ m^{-2} & \text { if } \ell \neq \ell^{\prime}, k=k^{\prime} \\ m^{-1} & \text { if } \ell \neq \ell^{\prime}, k \neq k^{\prime}, k=\ell^{\prime}, \ell=k^{\prime} \\ m^{-2} & \text { if } \ell \neq \ell^{\prime}, k \neq k^{\prime}, k=\ell^{\prime}, \ell \neq k^{\prime} \\ m^{-2} & \text { if } \ell \neq \ell^{\prime}, k \neq k^{\prime}, k \neq \ell^{\prime}, \ell=k^{\prime} \\ m^{-3} & \text { if } \ell \neq \ell^{\prime}, k \neq k^{\prime}, k \neq \ell^{\prime}, \ell \neq k^{\prime} .\end{cases}
$$

In addition, since there is one non-zero entry for each column in count sketch,

$$
\sum_{i=1}^{m} \sum_{i^{\prime}=1, i^{\prime} \neq i}^{m} \delta_{i k} \delta_{i \ell} \delta_{i^{\prime} k^{\prime}} \delta_{i^{\prime} \ell^{\prime}}= \begin{cases}0 & \text { if } \ell=\ell^{\prime}, k=k^{\prime} \\ 0 & \text { if } \ell=\ell^{\prime}, k \neq k^{\prime} \\ 0 & \text { if } \ell \neq \ell^{\prime}, k=k^{\prime} \\ 0 & \text { if } \ell \neq \ell^{\prime}, k \neq k^{\prime}, k=\ell^{\prime}, \ell=k^{\prime} \\ 0 & \text { if } \ell \neq \ell^{\prime}, k \neq k^{\prime}, k=\ell^{\prime}, \ell \neq k^{\prime} \\ 0 & \text { if } \ell \neq \ell^{\prime}, k \neq k^{\prime}, k \neq \ell^{\prime}, \ell=k^{\prime} \\ \sum_{i=1}^{m} \sum_{i^{\prime}=1, i^{\prime} \neq i}^{m} \delta_{i k} \delta_{i \ell} \delta_{i^{\prime} k^{\prime}} \delta_{i^{\prime} \ell^{\prime}} & \text { if } \ell \neq \ell^{\prime}, k \neq k^{\prime}, k \neq \ell^{\prime}, \ell \neq k^{\prime} .\end{cases}
$$

Hence,

$$
\mathbb{E}\left[\sum_{i=1}^{m} \sum_{i^{\prime}=1, i^{\prime} \neq i}^{m} \delta_{i k} \delta_{i \ell} \delta_{i^{\prime} k^{\prime}} \delta_{i^{\prime} \ell^{\prime}}\right]= \begin{cases}0 & \text { if } \ell=\ell^{\prime}, k=k^{\prime} \\ 0 & \text { if } \ell=\ell^{\prime}, k \neq k^{\prime} \\ 0 & \text { if } \ell \neq \ell^{\prime}, k=k^{\prime} \\ 0 & \text { if } \ell \neq \ell^{\prime}, k \neq k^{\prime}, k=\ell^{\prime}, \ell=k^{\prime} \\ 0 & \text { if } \ell \neq \ell^{\prime}, k \neq k^{\prime}, k=\ell^{\prime}, \ell \neq k^{\prime} \\ 0 & \text { if } \ell \neq \ell^{\prime}, k \neq k^{\prime}, k \neq \ell^{\prime}, \ell=k^{\prime} \\ \frac{m-1}{m^{3}} & \text { if } \ell \neq \ell^{\prime}, k \neq k^{\prime}, k \neq \ell^{\prime}, \ell \neq k^{\prime} .\end{cases}
$$

Combining the results above yields

$$
\mathbb{E}\left[k_{m}(k, \ell) k_{m}\left(k^{\prime}, \ell^{\prime}\right)\right]= \begin{cases}\frac{m-1}{m^{2}} & \text { if } \ell=\ell^{\prime}, k=k^{\prime} \\ 0 & \text { if } \ell=\ell^{\prime}, k \neq k^{\prime} \\ 0 & \text { if } \ell \neq \ell^{\prime}, k=k^{\prime} \\ \frac{m-1}{m^{2}} & \text { if } \ell \neq \ell^{\prime}, k \neq k^{\prime}, k=\ell^{\prime}, \ell=k^{\prime} \\ 0 & \text { if } \ell \neq \ell^{\prime}, k \neq k^{\prime}, k=\ell^{\prime}, \ell \neq k^{\prime} \\ 0 & \text { if } \ell \neq \ell^{\prime}, k \neq k^{\prime}, k \neq \ell^{\prime}, \ell=k^{\prime} \\ 0 & \text { if } \ell \neq \ell^{\prime}, k \neq k^{\prime}, k \neq \ell^{\prime}, \ell \neq k^{\prime} .\end{cases}
$$


Note that

$$
\begin{aligned}
\mathbb{E}\left[T_{1, u v}^{2}\right] & =\sum_{\ell=1}^{n} \sum_{\ell^{\prime}=1}^{n} \sum_{k=1, k \neq \ell}^{n} \sum_{k^{\prime}=1, k^{\prime} \neq \ell^{\prime}}^{n} \Psi_{k k} \Psi_{k^{\prime} k^{\prime}} \mathbb{E}\left[k_{m}(k, \ell) k_{m}\left(k^{\prime}, \ell^{\prime}\right)\right] U_{\ell u} U_{\ell v} U_{\ell^{\prime} u} U_{\ell^{\prime} v} \\
& =\frac{m-1}{m^{2}} \sum_{\ell=1}^{n} \sum_{k=1, k \neq \ell}^{n} \Psi_{k k}^{2} U_{\ell u}^{2} U_{\ell v}^{2}+\frac{m-1}{m^{2}} \sum_{\ell=1}^{n} \Psi_{\ell \ell} U_{\ell u} U_{\ell v} \sum_{\ell^{\prime}=1, \ell^{\prime} \neq \ell}^{n} \Psi_{\ell^{\prime} \ell^{\prime}} U_{\ell^{\prime} u} U_{\ell^{\prime} v} \\
& =\frac{m-1}{m^{2}} \sum_{\ell=1}^{n}\left[\operatorname{tr}\left(\Psi^{2}\right)-\Psi_{\ell \ell}^{2}\right] U_{\ell u}^{2} U_{\ell v}^{2} \\
& +\frac{m-1}{m^{2}} \sum_{\ell=1}^{n} \Psi_{\ell \ell} U_{\ell u} U_{\ell v}\left[\sum_{\ell^{\prime}=1}^{n} \Psi_{\ell^{\prime} \ell^{\prime}} U_{\ell^{\prime} u} U_{\ell^{\prime} v}-\Psi_{\ell \ell} U_{\ell u} U_{\ell v}\right] \\
& \leq \frac{m-1}{m^{2}}\left\{\operatorname{tr}\left(\Psi^{2}\right)+\left[U^{T} \Psi U\right]_{u v}^{2}\right\}
\end{aligned}
$$

Therefore,

$$
\begin{aligned}
& \sum_{u=1}^{d} \sum_{v=1}^{d} \mathbb{E}\left[T_{1, u v}^{2}\right] \\
& \leq \frac{d^{2}(m-1)}{m^{2}} \operatorname{tr}\left(\Psi^{2}\right)+\frac{m-1}{m^{2}} \sum_{u=1}^{d} \sum_{v=1}^{d}\left[U^{T} \Psi U\right]_{u v}^{2} \\
& =\frac{d^{2}(m-1)}{m^{2}} \operatorname{tr}\left(\Psi^{2}\right)+\frac{m-1}{m^{2}}\left\|U^{T} \Psi U\right\|_{F}^{2} \\
& =\frac{2 d^{2}(m-1)}{m^{2}} \operatorname{tr}\left(\Psi^{2}\right) .
\end{aligned}
$$

Now consider $T_{2, u v}$. Recall that

$$
T_{2, u v}=\sum_{\ell=1}^{n} \sum_{\ell^{\prime}=1, \ell^{\prime} \neq \ell}^{n} \sum_{k=1}^{n} \Psi_{k k}\left[\sum_{i=1}^{m} \delta_{i k} \delta_{i \ell} \delta_{i \ell^{\prime}} \sigma_{i \ell} \sigma_{i \ell^{\prime}}\right] U_{\ell u} U_{\ell^{\prime} v}
$$

Define

$$
q_{n, m}\left(\ell, \ell^{\prime}\right):=\sum_{k=1}^{n} \Psi_{k k}\left[\sum_{i=1}^{m} \delta_{i k} \delta_{i \ell} \delta_{i \ell^{\prime}} \sigma_{i \ell} \sigma_{i \ell^{\prime}}\right]
$$

Note that

$$
\mathbb{E}\left[T_{2, u v}^{2}\right]=\sum_{\ell=1}^{n} \sum_{\tilde{\ell}=1}^{n} \sum_{\ell^{\prime}=1, \ell^{\prime} \neq \ell{\widetilde{\ell^{\prime}}}_{1}=1, \widetilde{\ell}^{\prime} \neq \tilde{\ell}}^{n} \mathbb{E}\left[q_{n, m}\left(\ell, \ell^{\prime}\right) q_{n, m}\left(\widetilde{\ell}, \widetilde{\ell}^{\prime}\right)\right] U_{\ell u} U_{\ell^{\prime} v} U_{\widetilde{\ell} u} U_{\widetilde{\ell}^{\prime} v} .
$$

Write

$$
\begin{aligned}
& q_{n, m}\left(\ell, \ell^{\prime}\right) q_{n, m}\left(\widetilde{\ell}, \widetilde{\ell^{\prime}}\right) \\
& =\sum_{k=1}^{n} \sum_{k^{\prime}=1}^{n} \sum_{i=1}^{m} \sum_{i^{\prime}=1}^{m} \Psi_{k k} \Psi_{k^{\prime} k^{\prime}} \delta_{i k} \delta_{i \ell} \delta_{i \ell^{\prime}} \delta_{i^{\prime} k^{\prime}} \delta_{i^{\prime} \ell^{\prime}} \delta_{i^{\prime} \widetilde{\ell}^{\prime}} \sigma_{i \ell} \sigma_{i \ell^{\prime}} \sigma_{i^{\prime} \widetilde{\ell}^{\prime}} \sigma_{i^{\prime} \widetilde{\ell}^{\prime}} .
\end{aligned}
$$


Then for any $\ell \neq \ell^{\prime}$ and $\widetilde{\ell} \neq \widetilde{\ell^{\prime}}$,

$$
\begin{aligned}
& \mathbb{E}\left[q_{n, m}\left(\ell, \ell^{\prime}\right) q_{n, m}\left(\widetilde{\ell}, \widetilde{\ell}^{\prime}\right)\right] \\
& = \begin{cases}\sum_{k=1}^{n} \sum_{k^{\prime}=1}^{n} \sum_{i=1}^{m} \Psi_{k k} \Psi_{k^{\prime} k^{\prime}} \mathbb{E}\left[\delta_{i k} \delta_{i \ell} \delta_{i \ell^{\prime}} \delta_{i k^{\prime}}\right] & \text { if } \ell=\widetilde{\ell}, \ell^{\prime}=\widetilde{\ell^{\prime}} \\
\sum_{k=1}^{n} \sum_{k^{\prime}=1}^{n} \sum_{i=1}^{m} \Psi_{k k} \Psi_{k^{\prime} k^{\prime}} \mathbb{E}\left[\delta_{i k} \delta_{i \ell} \delta_{i \ell^{\prime}} \delta_{i k^{\prime}}\right] & \text { if } \ell=\widetilde{\ell^{\prime}}, \ell^{\prime}=\widetilde{\ell} \\
0 & \text { otherwise. }\end{cases}
\end{aligned}
$$

Thus,

$$
\begin{aligned}
\mathbb{E}\left[T_{2, u v}^{2}\right] & =\sum_{\ell=1}^{n} \sum_{\ell^{\prime}=1, \ell^{\prime} \neq \ell}^{n} \mathbb{E}\left[q_{n, m}^{2}\left(\ell, \ell^{\prime}\right)\right] U_{\ell u}^{2} U_{\ell^{\prime} v}^{2} \\
& +\sum_{\ell=1}^{n} \sum_{\ell^{\prime}=1, \ell^{\prime} \neq \ell}^{n} \mathbb{E}\left[q_{n, m}\left(\ell, \ell^{\prime}\right) q_{n, m}\left(\ell^{\prime}, \ell\right)\right] U_{\ell u} U_{\ell^{\prime} v} U_{\ell^{\prime} u} U_{\ell v}
\end{aligned}
$$

Further, write

$$
\begin{aligned}
& \sum_{k=1}^{n} \sum_{k^{\prime}=1}^{n} \sum_{i=1}^{m} \Psi_{k k} \Psi_{k^{\prime} k^{\prime}} \mathbb{E}\left[\delta_{i k} \delta_{i \ell} \delta_{i \ell^{\prime}} \delta_{i k^{\prime}}\right] \\
& =\sum_{k=1}^{n} \sum_{i=1}^{m} \Psi_{k k}^{2} \mathbb{E}\left[\delta_{i k} \delta_{i \ell} \delta_{i \ell^{\prime}}\right]+\sum_{k=1}^{n} \sum_{k^{\prime}=1, k^{\prime} \neq k}^{n} \sum_{i=1}^{m} \Psi_{k k} \Psi_{k^{\prime} k^{\prime}} \mathbb{E}\left[\delta_{i k} \delta_{i \ell} \delta_{i \ell^{\prime}} \delta_{i k^{\prime}}\right] .
\end{aligned}
$$

Note that for any $\ell \neq \ell^{\prime}$,

$$
\mathbb{E}\left[\delta_{i k} \delta_{i \ell} \delta_{i \ell^{\prime}}\right]= \begin{cases}\frac{1}{m^{2}} & \text { if } k=\ell \text { or } k=\ell^{\prime} \\ \frac{1}{m^{3}} & \text { otherwise }\end{cases}
$$

so that

$$
\begin{aligned}
\sum_{k=1}^{n} \sum_{i=1}^{m} \Psi_{k k}^{2} \mathbb{E}\left[\delta_{i k} \delta_{i \ell} \delta_{i \ell^{\prime}}\right] & =\left[\frac{2}{m^{2}}+\frac{m-2}{m^{3}}\right] \operatorname{tr}\left(\Psi^{2}\right) \\
& =\sum_{k=1}^{n} \Psi_{k k}^{2}\left[1\left(k=\ell \text { or } k=\ell^{\prime}\right) \frac{1}{m}+1\left(k \neq \ell \text { and } k \neq \ell^{\prime}\right) \frac{1}{m^{2}}\right] \\
& \leq \frac{\operatorname{tr}\left(\Psi^{2}\right)}{m} .
\end{aligned}
$$

Similarly, for any $\ell \neq \ell^{\prime}$ and $k \neq k^{\prime}$,

$$
\mathbb{E}\left[\delta_{i k} \delta_{i \ell} \delta_{i \ell^{\prime}} \delta_{i k^{\prime}}\right]= \begin{cases}\frac{1}{m^{2}} & \text { if } k=\ell, k^{\prime}=\ell^{\prime} \\ \frac{1}{m^{2}} & \text { if } k=\ell^{\prime}, k^{\prime}=\ell \\ \frac{1}{m^{3}} & \text { if } k=\ell, k^{\prime} \neq \ell^{\prime} \\ \frac{1}{m^{3}} & \text { if } k \neq \ell, k^{\prime}=\ell^{\prime} \\ \frac{1}{m^{4}} & \text { if } k \neq \ell, k^{\prime} \neq \ell^{\prime}\end{cases}
$$


Thus,

$$
\begin{aligned}
& \sum_{k=1}^{n} \sum_{k^{\prime}=1, k^{\prime} \neq k}^{n} \sum_{i=1}^{m} \Psi_{k k} \Psi_{k^{\prime} k^{\prime}} \mathbb{E}\left[\delta_{i k} \delta_{i \ell} \delta_{i \ell^{\prime}} \delta_{i k^{\prime}}\right] \\
& =\frac{2}{m} \Psi_{\ell \ell} \Psi_{\ell^{\prime} \ell^{\prime}}+\frac{1}{m^{2}} \Psi_{\ell \ell} \sum_{k^{\prime}=1, k^{\prime} \neq \ell, k^{\prime} \neq \ell^{\prime}}^{n} \Psi_{k^{\prime} k^{\prime}}+\frac{1}{m^{2}} \Psi_{\ell^{\prime} \ell^{\prime}} \sum_{k=1, k \neq \ell^{\prime}, k \neq \ell}^{n} \Psi_{k k} \\
& +\frac{1}{m^{3}} \sum_{k=1, k \neq \ell}^{n} \sum_{k^{\prime}=1, k^{\prime} \neq k, k^{\prime} \neq \ell^{\prime}}^{n} \Psi_{k k} \Psi_{k^{\prime} k^{\prime}} \\
& =\frac{2}{m} \Psi_{\ell \ell} \Psi_{\ell^{\prime} \ell^{\prime}}+\frac{1}{m^{2}}\left(\Psi_{\ell \ell}+\Psi_{\ell^{\prime} \ell^{\prime}}\right)\left[\operatorname{tr}(\Psi)-\Psi_{\ell \ell}-\Psi_{\ell^{\prime} \ell^{\prime}}\right] \\
& +\frac{1}{m^{3}}\left\{\left[\operatorname{tr}(\Psi)-\Psi_{\ell \ell}\right]\left[\operatorname{tr}(\Psi)-\Psi_{\ell^{\prime} \ell^{\prime}}\right]-\left[\operatorname{tr}\left(\Psi^{2}\right)-\Psi_{\ell \ell}^{2}\right]\right\} \\
& =\frac{2}{m} \Psi_{\ell \ell} \Psi_{\ell^{\prime} \ell^{\prime}}-\frac{1}{m^{2}}\left(\Psi_{\ell \ell}+\Psi_{\ell^{\prime} \ell^{\prime}}\right)^{2} \\
& +\frac{1}{m^{2}}\left(\Psi_{\ell \ell}+\Psi_{\ell^{\prime} \ell^{\prime}}\right) \operatorname{tr}(\Psi)-\frac{1}{m^{3}}\left(\Psi_{\ell \ell}+\Psi_{\ell^{\prime} \ell^{\prime}}\right) \operatorname{tr}(\Psi)-\frac{1}{m^{3}} \operatorname{tr}\left(\Psi^{2}\right) \\
& +\frac{1}{m^{3}}\left\{[\operatorname{tr}(\Psi)]^{2}+\Psi_{\ell \ell} \Psi_{\ell^{\prime} \ell^{\prime}}+\Psi_{\ell \ell}^{2}\right\} \\
& \leq \frac{2}{m} \Psi_{\ell \ell} \Psi_{\ell^{\prime} \ell^{\prime}}+\frac{1}{m^{2}}\left(\Psi_{\ell \ell}+\Psi_{\ell^{\prime} \ell^{\prime}}\right) \operatorname{tr}(\Psi)+\frac{1}{m^{3}}\left\{[\operatorname{tr}(\Psi)]^{2}+\Psi_{\ell \ell} \Psi_{\ell^{\prime} \ell^{\prime}}+\Psi_{\ell \ell}^{2}\right\} .
\end{aligned}
$$

Note that

$$
\begin{gathered}
\sum_{\ell=1}^{n} \sum_{\ell^{\prime}=1, \ell^{\prime} \neq \ell}^{n} \Psi_{\ell \ell} \Psi_{\ell^{\prime} \ell^{\prime}} U_{\ell u}^{2} U_{\ell^{\prime} v}^{2} \\
\sum_{\ell=1}^{n} \sum_{\ell^{\prime}=1, \ell^{\prime} \neq \ell}^{n} \Psi_{\ell \ell} U_{\ell u}^{2} U_{\ell^{\prime} v}^{2} \leq\|\Psi\|_{2}, \\
\sum_{\ell=1}^{n} \sum_{\ell^{\prime}=1, \ell^{\prime} \neq \ell}^{n} \Psi_{\ell^{\prime} \ell^{\prime}} U_{\ell u}^{2} U_{\ell^{\prime} v}^{2} \leq\|\Psi\|_{2}, \\
\sum_{\ell=1}^{n} \sum_{\ell^{\prime}=1, \ell^{\prime} \neq \ell}^{n} \Psi_{\ell \ell}^{2} U_{\ell u}^{2} U_{\ell^{\prime} v}^{2} \leq\|\Psi\|_{2}^{2}, \\
\sum_{\ell=1}^{n} \sum_{\ell^{\prime}=1, \ell^{\prime} \neq \ell}^{n} U_{\ell u}^{2} U_{\ell^{\prime} v}^{2} \leq 1 .
\end{gathered}
$$

Using these to obtain

$$
\begin{aligned}
& \sum_{\ell=1}^{n} \sum_{\ell^{\prime}=1, \ell^{\prime} \neq \ell}^{n} \mathbb{E}\left[q_{n, m}^{2}\left(\ell, \ell^{\prime}\right)\right] U_{\ell u}^{2} U_{\ell^{\prime} v}^{2} \\
& \leq \frac{2}{m}\|\Psi\|_{2}^{2}+\frac{2}{m^{2}}\|\Psi\|_{2} \operatorname{tr}(\Psi)+\frac{1}{m^{3}}\left\{[\operatorname{tr}(\Psi)]^{2}+2\|\Psi\|_{2}^{2}\right\} .
\end{aligned}
$$


Now

$$
\begin{aligned}
& \sum_{\ell=1}^{n} \sum_{\ell^{\prime}=1, \ell^{\prime} \neq \ell}^{n} \Psi_{\ell \ell} \Psi_{\ell^{\prime} \ell^{\prime}} U_{\ell u} U_{\ell^{\prime} v} U_{\ell^{\prime} u} U_{\ell v} \\
& =\sum_{\ell=1}^{n} \Psi_{\ell \ell} U_{\ell u} U_{\ell v}\left[\sum_{\ell^{\prime}=1, \ell^{\prime} \neq \ell}^{n} \Psi_{\ell^{\prime} \ell^{\prime}} U_{\ell^{\prime} v} U_{\ell^{\prime} u}\right] \\
& =\sum_{\ell=1}^{n} \Psi_{\ell \ell} U_{\ell u} U_{\ell v}\left\{\left[U^{T} \Psi U\right]_{u v}-\Psi_{\ell \ell} U_{\ell v} U_{\ell u}\right\} \\
& \leq\left[U^{T} \Psi U\right]_{u v}^{2} .
\end{aligned}
$$

Similarly,

$$
\begin{aligned}
& \sum_{\ell=1}^{n} \sum_{\ell^{\prime}=1, \ell^{\prime} \neq \ell}^{n} \Psi_{\ell \ell} U_{\ell u} U_{\ell^{\prime} v} U_{\ell^{\prime} u} U_{\ell v} \leq\left[U^{T} \Psi U\right]_{u v}\left[U^{T} U\right]_{u v}, \\
& \sum_{\ell=1}^{n} \sum_{\ell^{\prime}=1, \ell^{\prime} \neq \ell}^{n} \Psi_{\ell^{\prime} \ell^{\prime}} U_{\ell u} U_{\ell^{\prime} v} U_{\ell^{\prime} u} U_{\ell v} \leq\left[U^{T} \Psi U\right]_{u v}\left[U^{T} U\right]_{u v}, \\
& \sum_{\ell=1}^{n} \sum_{\ell^{\prime}=1, \ell^{\prime} \neq \ell}^{n} \Psi_{\ell \ell}^{2} U_{\ell u} U_{\ell^{\prime} v} U_{\ell^{\prime} u} U_{\ell v} \leq\left[U^{T} \Psi^{2} U\right]_{u v}\left[U^{T} U\right]_{u v}, \\
& \sum_{\ell=1}^{n} \sum_{\ell^{\prime}=1, \ell^{\prime} \neq \ell}^{n} U_{\ell u} U_{\ell^{\prime} v} U_{\ell^{\prime} u} U_{\ell v} \leq\left[U^{T} U\right]_{u v} .
\end{aligned}
$$

Then

$$
\begin{aligned}
& \sum_{\ell=1}^{n} \sum_{\ell^{\prime}=1, \ell^{\prime} \neq \ell}^{n} \mathbb{E}\left[q_{n, m}\left(\ell, \ell^{\prime}\right) q_{n, m}\left(\ell^{\prime}, \ell\right)\right] U_{\ell u} U_{\ell^{\prime} v} U_{\ell^{\prime} u} U_{\ell v} \\
& \leq \frac{2}{m}\left[U^{T} \Psi U\right]_{u v}^{2}+\frac{2}{m^{2}}\left[U^{T} \Psi U\right]_{u v}\left[U^{T} U\right]_{u v} \\
& +\frac{1}{m^{3}}\left\{[\operatorname{tr}(\Psi)]^{2}\left[U^{T} U\right]_{u v}+\left[U^{T} \Psi U\right]_{u v}^{2}+\left[U^{T} \Psi^{2} U\right]_{u v}\left[U^{T} U\right]_{u v}\right\} .
\end{aligned}
$$

Combing the results above together gives

$$
\begin{aligned}
& \mathbb{E}\left[T_{2, u v}^{2}\right] \\
& \leq \frac{2}{m}\|\Psi\|_{2}^{2}+\frac{2}{m^{2}}\|\Psi\|_{2} \operatorname{tr}(\Psi)+\frac{1}{m^{3}}\left\{[\operatorname{tr}(\Psi)]^{2}+2\|\Psi\|_{2}^{2}\right\} \\
& +\frac{2}{m}\left[U^{T} \Psi U\right]_{u v}^{2}+\frac{2}{m^{2}}\left[U^{T} \Psi U\right]_{u v}\left[U^{T} U\right]_{u v} \\
& +\frac{1}{m^{3}}\left\{[\operatorname{tr}(\Psi)]^{2}\left[U^{T} U\right]_{u v}+\left[U^{T} \Psi U\right]_{u v}^{2}+\left[U^{T} \Psi^{2} U\right]_{u v}\left[U^{T} U\right]_{u v}\right\} .
\end{aligned}
$$


Thus,

$$
\begin{aligned}
& \sum_{u=1}^{d} \sum_{v=1}^{d} \mathbb{E}\left[T_{2, u v}^{2}\right] \\
& \leq \frac{2 d^{2}}{m}\|\Psi\|_{2}^{2}+\frac{2 d^{2}}{m^{2}}\|\Psi\|_{2} \operatorname{tr}(\Psi)+\frac{d^{2}}{m^{3}}\left\{[\operatorname{tr}(\Psi)]^{2}+2\|\Psi\|_{2}^{2}\right\} \\
& +\frac{2}{m} \operatorname{tr}\left(\Psi^{2}\right)+\frac{2}{m^{2}} \operatorname{tr}(\Psi)+\frac{1}{m^{3}}\left\{d[\operatorname{tr}(\Psi)]^{2}+2 \operatorname{tr}\left(\Psi^{2}\right)\right\} .
\end{aligned}
$$

Choose $A(\Psi, m, n)$ as in $(22)$. Then

$$
\begin{aligned}
& \mathbb{P}\left(\left\|U^{T} \Pi^{T} \Pi \Psi \Pi^{T} \Pi U-U^{T} A(\Psi, m, n) U\right\|_{2}>\epsilon\right) \\
& \leq \epsilon^{-2} \mathbb{E}\left[\left\|U^{T} \Pi^{T} \Pi \Psi \Pi^{T} \Pi U-U^{T} A(\Psi, m, n) U\right\|_{F}^{2}\right] \\
& \leq 2 \epsilon^{-2} \sum_{u=1}^{d} \sum_{v=1}^{d} \mathbb{E}\left[T_{1, u v}^{2}+T_{2, u v}^{2}\right] \\
& =2 \epsilon^{-2}\left\{\frac{2 d^{2}(m-1)}{m^{2}} \operatorname{tr}\left(\Psi^{2}\right)+\frac{2 d^{2}}{m^{2}}\|\Psi\|_{2} \operatorname{tr}(\Psi)+\frac{d^{2}}{m^{3}}\left\{[\operatorname{tr}(\Psi)]^{2}+2\|\Psi\|_{2}^{2}\right\}\right. \\
& \left.\quad+\frac{2}{m} \operatorname{tr}\left(\Psi^{2}\right)+\frac{2}{m^{2}} \operatorname{tr}(\Psi)+\frac{1}{m^{3}}\left\{d[\operatorname{tr}(\Psi)]^{2}+2 \operatorname{tr}\left(\Psi^{2}\right)\right\}\right\} .
\end{aligned}
$$

Q.E.D. 
Table 1: Assessment of JL Lemma: $n=20,000, d=5$.

\begin{tabular}{|c|c|c|c|c|c|c|c|c|c|}
\hline \multirow[t]{2}{*}{$m$} & \multicolumn{3}{|c|}{ Random Sampling } & \multicolumn{6}{|c|}{ Random Projections } \\
\hline & RS1 & $\mathrm{RS} 2$ & RS3 & RP1 & RP2 & RP3 & $\mathrm{RP} 4$ & $\mathrm{CS}$ & LEV \\
\hline Normal & \multicolumn{9}{|c|}{ Norm approximation } \\
\hline 161 & 0.627 & 0.624 & 0.538 & 0.628 & 0.633 & 0.631 & 0.640 & 0.642 & 0.757 \\
\hline 322 & 0.801 & 0.792 & 0.700 & 0.790 & 0.795 & 0.795 & 0.800 & 0.793 & 0.909 \\
\hline 644 & 0.931 & 0.931 & 0.871 & 0.926 & 0.929 & 0.927 & 0.931 & 0.928 & 0.982 \\
\hline 966 & 0.978 & 0.972 & 0.932 & 0.971 & 0.974 & 0.974 & 0.975 & 0.972 & 0.997 \\
\hline 1288 & 0.990 & 0.987 & 0.973 & 0.990 & 0.991 & 0.989 & 0.990 & 0.991 & 1.000 \\
\hline \multirow[t]{2}{*}{2576} & 1.000 & 1.000 & 0.998 & 1.000 & 1.000 & 1.000 & 1.000 & 1.000 & 1.000 \\
\hline & \multicolumn{9}{|c|}{ Eigenvalue distortion } \\
\hline 161 & 0.189 & 0.191 & 0.191 & 0.189 & 0.187 & 0.188 & 0.189 & 0.188 & 0.158 \\
\hline 322 & 0.126 & 0.128 & 0.127 & 0.127 & 0.127 & 0.128 & 0.126 & 0.129 & 0.105 \\
\hline 644 & 0.082 & 0.085 & 0.084 & 0.086 & 0.084 & 0.085 & 0.085 & 0.086 & 0.071 \\
\hline 966 & 0.065 & 0.067 & 0.065 & 0.067 & 0.066 & 0.067 & 0.067 & 0.068 & 0.055 \\
\hline 1288 & 0.055 & 0.056 & 0.055 & 0.056 & 0.056 & 0.056 & 0.055 & 0.055 & 0.045 \\
\hline 2576 & 0.033 & 0.036 & 0.033 & 0.036 & 0.037 & 0.036 & 0.035 & 0.037 & 0.029 \\
\hline Exponential & \multicolumn{9}{|c|}{ "Norm approximation } \\
\hline 161 & 0.432 & 0.429 & 0.402 & 0.627 & 0.624 & 0.636 & 0.628 & 0.637 & 0.717 \\
\hline 322 & 0.580 & 0.578 & 0.548 & 0.796 & 0.795 & 0.794 & 0.800 & 0.791 & 0.875 \\
\hline 644 & 0.747 & 0.738 & 0.717 & 0.925 & 0.930 & 0.929 & 0.930 & 0.928 & 0.972 \\
\hline 966 & 0.851 & 0.840 & 0.812 & 0.971 & 0.968 & 0.973 & 0.969 & 0.972 & 0.992 \\
\hline 1288 & 0.899 & 0.894 & 0.866 & 0.990 & 0.988 & 0.989 & 0.991 & 0.989 & 0.998 \\
\hline \multirow[t]{2}{*}{2576} & 0.986 & 0.974 & 0.975 & 1.000 & 1.000 & 1.000 & 1.000 & 1.000 & 1.000 \\
\hline & \multicolumn{9}{|c|}{ Eigenvalue distortion } \\
\hline 161 & 0.263 & 0.257 & 0.259 & 0.188 & 0.193 & 0.188 & 0.190 & 0.188 & 0.158 \\
\hline 322 & 0.176 & 0.177 & 0.175 & 0.126 & 0.128 & 0.127 & 0.127 & 0.127 & 0.104 \\
\hline 644 & 0.116 & 0.118 & 0.116 & 0.084 & 0.083 & 0.083 & 0.082 & 0.085 & 0.069 \\
\hline 966 & 0.090 & 0.094 & 0.090 & 0.066 & 0.067 & 0.066 & 0.065 & 0.065 & 0.055 \\
\hline 1288 & 0.076 & 0.079 & 0.075 & 0.055 & 0.055 & 0.055 & 0.054 & 0.055 & 0.045 \\
\hline 2576 & 0.048 & 0.052 & 0.048 & 0.036 & 0.036 & 0.037 & 0.035 & 0.036 & 0.030 \\
\hline
\end{tabular}


Table 2: Monte Carlo Experiments: Properties of Combining Sketches, $n=1 e 6$

\begin{tabular}{|c|c|c|c|c|c|c|c|c|c|}
\hline$m$ & $J$ & RS1 & SRHT & CS & $\begin{array}{l}=3 \\
\mathrm{LEV}\end{array}$ & RS1 & SRHT & CS & LEV \\
\hline & & \multicolumn{4}{|c|}{$\hat{\beta}_{3}$ with $\beta_{3}=1.0$} & \multicolumn{4}{|c|}{$\operatorname{se}\left(\hat{\beta}_{3}\right)$} \\
\hline 500 & 1 & 0.999 & 1.002 & 0.999 & 1.000 & 0.046 & 0.044 & 0.045 & 0.039 \\
\hline 500 & 5 & 0.999 & 1.000 & 1.000 & 1.001 & 0.021 & 0.020 & 0.020 & 0.018 \\
\hline 500 & 10 & 1.000 & 1.000 & 1.000 & 1.000 & 0.014 & 0.015 & 0.015 & 0.012 \\
\hline 1000 & 1 & 1.000 & 0.999 & 0.998 & 1.000 & 0.032 & 0.032 & 0.031 & 0.026 \\
\hline 1000 & 5 & 1.000 & 1.000 & 1.000 & 1.000 & 0.014 & 0.015 & 0.015 & 0.012 \\
\hline 1000 & 10 & 1.000 & 0.999 & 1.000 & 1.000 & 0.010 & 0.010 & 0.010 & 0.008 \\
\hline 2000 & 1 & 1.001 & 1.000 & 1.001 & 1.000 & 0.021 & 0.022 & 0.022 & 0.019 \\
\hline 2000 & 5 & 1.000 & 1.000 & 1.000 & 1.000 & 0.010 & 0.010 & 0.010 & 0.008 \\
\hline 2000 & 10 & 1.000 & 1.000 & 1.000 & 1.000 & 0.007 & 0.007 & 0.007 & 0.006 \\
\hline 5000 & 1 & 1.000 & 1.001 & 0.999 & 1.000 & 0.014 & 0.014 & 0.014 & 0.012 \\
\hline 5000 & 5 & 1.000 & 1.000 & 0.999 & 1.000 & 0.006 & 0.006 & 0.006 & 0.005 \\
\hline \multirow[t]{2}{*}{5000} & 10 & 1.000 & 1.000 & 1.000 & 1.000 & 0.005 & 0.005 & 0.004 & 0.004 \\
\hline & & \multicolumn{4}{|c|}{ Size } & \multicolumn{4}{|c|}{ Power, $\beta_{3}=0.98$} \\
\hline 500 & 1 & 0.050 & 0.040 & 0.062 & 0.063 & 0.081 & 0.069 & 0.071 & 0.104 \\
\hline 500 & 5 & 0.035 & 0.029 & 0.021 & 0.045 & 0.114 & 0.115 & 0.123 & 0.185 \\
\hline 500 & 10 & 0.039 & 0.051 & 0.053 & 0.037 & 0.276 & 0.258 & 0.265 & 0.345 \\
\hline 1000 & 1 & 0.048 & 0.044 & 0.050 & 0.052 & 0.101 & 0.101 & 0.085 & 0.113 \\
\hline 1000 & 5 & 0.024 & 0.046 & 0.032 & 0.023 & 0.221 & 0.218 & 0.233 & 0.320 \\
\hline 1000 & 10 & 0.041 & 0.035 & 0.044 & 0.042 & 0.461 & 0.454 & 0.452 & 0.617 \\
\hline 2000 & 1 & 0.045 & 0.052 & 0.058 & 0.055 & 0.136 & 0.142 & 0.147 & 0.189 \\
\hline 2000 & 5 & 0.034 & 0.022 & 0.035 & 0.025 & 0.436 & 0.432 & 0.451 & 0.545 \\
\hline 2000 & 10 & 0.040 & 0.043 & 0.038 & 0.053 & 0.763 & 0.761 & 0.767 & 0.902 \\
\hline 5000 & 1 & 0.053 & 0.046 & 0.040 & 0.047 & 0.298 & 0.322 & 0.275 & 0.399 \\
\hline 5000 & 5 & 0.026 & 0.018 & 0.026 & 0.019 & 0.835 & 0.832 & 0.829 & 0.930 \\
\hline 5000 & 10 & 0.045 & 0.046 & 0.036 & 0.054 & 0.987 & 0.993 & 0.989 & 0.999 \\
\hline
\end{tabular}

\begin{tabular}{|c|c|c|c|c|c|c|c|c|c|}
\hline \multicolumn{10}{|c|}{$K=9$} \\
\hline$m$ & $J$ & RS1 & $\mathrm{CS}$ & RS1 & CS & RS1 & $\mathrm{CS}$ & RS1 & CS \\
\hline & & \multicolumn{2}{|c|}{ Size } & \multicolumn{2}{|c|}{ Power } & \multicolumn{2}{|c|}{$\hat{\beta}_{9}$} & \multicolumn{2}{|c|}{$\operatorname{se}\left(\hat{\beta}_{9}\right)$} \\
\hline 500 & 1 & 0.049 & 0.067 & 0.076 & 0.079 & 0.999 & 0.998 & 0.046 & 0.047 \\
\hline 500 & 5 & 0.038 & 0.029 & 0.129 & 0.120 & 1.000 & 1.000 & 0.021 & 0.020 \\
\hline 500 & 10 & 0.032 & 0.039 & 0.241 & 0.268 & 0.999 & 1.000 & 0.014 & 0.015 \\
\hline 1000 & 1 & 0.052 & 0.041 & 0.099 & 0.087 & 1.000 & 1.000 & 0.032 & 0.031 \\
\hline 1000 & 5 & 0.036 & 0.027 & 0.219 & 0.214 & 1.000 & 1.000 & 0.014 & 0.014 \\
\hline 1000 & 10 & 0.033 & 0.042 & 0.461 & 0.484 & 1.000 & 1.000 & 0.010 & 0.010 \\
\hline 2000 & 1 & 0.043 & 0.050 & 0.143 & 0.128 & 1.000 & 0.999 & 0.022 & 0.022 \\
\hline 2000 & 5 & 0.025 & 0.028 & 0.411 & 0.400 & 1.000 & 1.000 & 0.010 & 0.010 \\
\hline 2000 & 10 & 0.041 & 0.044 & 0.782 & 0.773 & 1.000 & 1.000 & 0.007 & 0.007 \\
\hline 5000 & 1 & 0.051 & 0.057 & 0.260 & 0.292 & 0.999 & 1.000 & 0.014 & 0.015 \\
\hline 5000 & 5 & 0.021 & 0.037 & 0.839 & 0.813 & 1.000 & 1.000 & 0.006 & 0.007 \\
\hline 5000 & 10 & 0.033 & 0.044 & 0.988 & 0.990 & 1.000 & 1.000 & 0.005 & 0.005 \\
\hline
\end{tabular}


Table 3: Inference Conscious Choice of $m$

\begin{tabular}{ll|lllll}
\multicolumn{6}{c}{$n=1 e 7, r=10, K=10, m_{0}=1000, \bar{\alpha}=0.05$} \\
\hline & & \multicolumn{5}{c}{$\left(\beta_{1}^{0}-\beta_{10}\right)$} \\
\hline $\bar{\gamma}$ & $\sigma_{e}$ & .005 & .01 & .015 & .02 & .025 \\
\hline 0.50 & 0.50 & 29686 & 7421 & 3298 & 1855 & 1187 \\
0.80 & 0.50 & 67837 & 16959 & 7537 & 4240 & 2713 \\
0.90 & 0.50 & 93965 & 23491 & 10441 & 5873 & 3759 \\
0.50 & 1.00 & 98296 & 24574 & 10922 & 6143 & 3932 \\
0.80 & 1.00 & 224620 & 56155 & 24958 & 14039 & 8985 \\
0.90 & 1.00 & 311136 & 77784 & 34571 & 19446 & 12445 \\
0.50 & 3.00 & 981128 & 245282 & 109014 & 61321 & 39245 \\
0.80 & 3.00 & 2242020 & 560505 & 249113 & 140126 & 89681 \\
0.90 & 3.00 & 3105562 & 776391 & 345062 & 194098 & 124222 \\
\hline
\end{tabular}


Table 4: Example of Belenzon et al. (2017)

\begin{tabular}{lcccc}
\hline \multirow{2}{*}{ Uniform Sampling } & $(1)$ & $(2)$ & $(3)$ & $(4)$ \\
\hline & Full Sample & $m_{1}$ & $m_{2}\left(m_{1}\right)$ & $m_{3}$ \\
\hline \multirow{2}{*}{ Dummy for eponymous } & $0.031^{* *}$ & $0.035^{* *}$ & $0.031^{* *}$ & $0.031^{* *}$ \\
& $(0.001)$ & $(0.010)$ & $(0.003)$ & $(0.002)$ \\
$\ln ($ assets) & $0.005^{* *}$ & 0.000 & $0.006^{* *}$ & $0.007^{* *}$ \\
& $(0.001)$ & $(0.004)$ & $(0.001)$ & $(0.001)$ \\
$\ln ($ no. shareholders) & $-0.032^{* *}$ & $-0.025^{* *}$ & $-0.030^{* *}$ & $-0.031^{* *}$ \\
Equity dispersion & $(0.001)$ & $(0.007)$ & $(0.002)$ & $(0.002)$ \\
& $-0.012^{* *}$ & -0.007 & $-0.016^{* *}$ & $-0.017^{* *}$ \\
& $(0.001)$ & $(0.011)$ & $(0.003)$ & $(0.003)$ \\
Omitted Covariates & & & & \\
Observations & & 132 & 58 & 54 \\
\hline
\end{tabular}

\begin{tabular}{lcccc}
\hline & $(1)$ & $(2)$ & $(3)$ & $(4)$ \\
Countsketch & Full Sample & $m_{1}$ & $m_{2}$ & $m_{3}$ \\
\hline \multirow{2}{*}{ Dummy for eponymous } & $0.031^{* *}$ & $0.035^{* *}$ & $0.030^{* *}$ & $0.032^{* *}$ \\
& $(0.001)$ & $(0.011)$ & $(0.003)$ & $(0.003)$ \\
$\ln ($ assets) & $0.005^{* *}$ & $0.009^{* *}$ & $0.004^{* *}$ & $0.005^{* *}$ \\
& $(0.001)$ & $(0.003)$ & $(0.001)$ & $(0.001)$ \\
$\ln$ (no. shareholders) & $-0.032^{* *}$ & $-0.028^{* *}$ & $-0.032^{* *}$ & $-0.032^{* *}$ \\
Equity dispersion & $(0.001)$ & $(0.008)$ & $(0.002)$ & $(0.002)$ \\
& $-0.012^{* *}$ & -0.024 & $-0.010^{*}$ & $-0.011^{* *}$ \\
Omitted Covariates & $(0.001)$ & $(0.014)$ & $(0.004)$ & $(0.004)$ \\
& & & & \\
Observations & & 2 & & 1 \\
\hline
\end{tabular}

Robust standard errors in parentheses

** $\mathrm{p}<0.01, * \mathrm{p}<0.05$ 


\section{References}

Achiloptas, D. 2003, Database Friendly Random Projections: Johnson-Lindenstrauss with Binary Coins, Journal of Computer and System Sciences 66(4), 671-687.

Agarwal, P. S. H.-P. and Varadarajan, K. 2004, Approximating Extent Measures of Points, Journal of the ACM 51(4), 606-635.

Ahfock, D., Astle, W. and Richardson, S. 2017, Statistical Properties of Sketching Algorithms, arXiv:1706.03665v1.

Ailon, N. and Chazelle, B. 2009, The Fast Johnson-Lindenstrauss Transform and Approximate Nearest Neighbors, SIAM Journal Computing 39(1), 302-322.

Alon, N., Matias, Y. and Onak, K. 1999, The Space Complexity of Approximating the Frequency Moments, Journal of Computational Systems Science 58(1), 137-147.

Bai, Z. and Yin, Y. 1993, Limit of the Smallest Eigenvalue of a Large Dimensional Sample Covariacne Matrix, Annals of Probability 21:3, 1275-1294.

Belenzon, S., Chatterji, A. and Dailey, B. 2017, Eponymous Enterpreneurs, American Economic Reivew 107:6, 1638-1655.

Boivin, J. and Ng, S. 2006, Are More Data Always Better for Factor Analysis, Journal of Econometrics 132, 169-194.

Boutidis, C. and Gittens, A. 2013, Improved Matrix Algorithms via the Subsampled Randomized Hadamadr Transform, SIAM Journal on Matrix Analysis 34:3, 1301-1340.

Breiman, L. 1999, Pasting Bites Together for Prediction in Large Data Sets and On-Line, Machine Learning 36(2), 85-103. https://www.stat.berkeley.edu/ breiman/pastebite.pdf.

Charika, M., Chen, K. and Farach-Colton, M. 2002, Finding Frequent Items in Data Streams, In Proceedings of International Colloquium on Automata, Languages, and Programming (ICALP) pp. 693-703.

Chawla, N., Hall, L., Bowyer, K. and Kegelmeyer, P. 2004, Learning Ensembles from Bites: A Scalable and Accurate Approach, Journal of Machine Learning Research 5, 421-451.

Chen, S., Varma, R., Singh, A. and Kovacevic, J. 2016, A Statistical Perspective of Sampling Scores for Linear Regresson, IEEE International Symposium on Information theory.

Chi, J. and Ipsen, I. 2018, Randomized Least Squares Regression: Combining Model and Algorithm Induced Uncertainties, xrXiv:1808.05924v1.

Christmann, A., Steinwart, I. and Hubert, M. 2007, Robust Learning from Bites for Data Mining, Computational Statistics and Data Analysis 52, 347-361.

Clarkson, K. and Woodruff, D. 2013, Low Rank Approximation and Regression in Input Sparsity Time, Proceedings of the 45th ACM Symposium on the Theory of Computing.

Cohen, M., Lee, Y., Musco, C., Musco, C., Peng, R. and Sidford, A. 2015, Uniform Sampling for Matrix Approximation, Proceedings of the 46th ACM Symposium on the Theory of Computing pp. 181-190.

Cohen, M., Nelson, J. and Woodruff, D. 2015, Optimal Approximate Matrix Product in Terms of Stable Rank, arXiv:1507.02268. 
Comrode, G., Garofalakis, M., Haas, P. and Jermaine, C. 2011, Synposes for Massive Data: Samples, Histograms, Wavelets,Sketches, Foundations and Trends in Databases 4(1), 1-294.

Conmode, G. and Muthukrishnan, S. 2005, An Improved Data Stream Summary: The Count-Min Sketch and Applications, Journal of Algorithms 55, 29-38.

Cramer, J. S. 1987, Mean and Variance of $R^{2}$ in Small and Moderate Samples, Journal of Econometrics 35, 253-266.

Dahiya, Y., Konomis, D. and Woodruff, D. 2018, An Empirical Evaluation of Sketching for Numerical Linear Algebra, KDD, http://www.cs.cmu.edu/afs/cs/user/dwoodruf/www/dkw18.pdf.

Dasgupta, S. and Gupta, A. 2003, An Elementary Proof of a Theorem of Johnson and Lindenstrauss, Random Structure and Algorithms 22(1), 60-65.

Dasgupta, A., Kumar, R. and Sarlos, T. 2010, A Sparse Lindenstrauss Transform, STOC pp. 341350 .

Deaton, A. and Ng, S. 1998, Parametric and Nonparametric Approaches to Tax Reform, Journal of the American Statistical Association 93(443), 900-909.

Dhillon, P., Lu, Y., Foster, D. and Ungar, L. 2013, New Subsampling Algorithms for Faster Least Squares Regression, Advances in Neural Information Processing Systems (NIPS) 26, 360-368.

Drineas, P. and Mahoney, M. 2005, On the Nystrom Method for Approximating a Gram Matrix for Improved Kernel Based Learning, Journal of Machine Learning Research 6, 2152-2125.

Drineas, P., Kannan, R. and Mahoney, M. 2006, Fast Monte Carlo Algorithms for Matrices I: Approximating Matrix Multiplications, SIAM Journal on Computing 36, 132-157.

Drineas, P., Magdon-Ismail, M., Mahoney, M. and Woodruff, D. 2012, Fast Approximation of Matrix Coherence and Statistical Leverage, Journal of Machine Learning Research 13, 34413472 .

Drineas, P., Mahoney, M. and Muthukrishnan, S. 2006, Sampling Algorithms for L2 Regression and Applications, Proceedings of the 17th Annual ACM-SIAM Symposium on Discrete Algorithms pp. 1127-1136.

Drineas, P., Mahoney, M., Muthukrishnan, S. and Sarlos, T. 2011, Faster Least Squares Approximation, Numerical Mathematics 117, 219-249.

Du Mouchel, W., Volinsky, C., Johnson, T., Cortes, C. and Pregibon, D. 1999, Squashing Flat Files Flatter, Proceedings of the Fifth ACM Conference on Knowledge Discovery and Data Mining pp. $6-15$.

Eriksson-Bique, S., Solberg, M., Stefanelli, M., Warkentin, S., Abbey, R. and Ipsen, I. 2011, Importance Sampling for a Monte Carlo Matrix Multiplication Algorithm with Application to Information Retrieval, SIAM Journal Computing 33(4), 1689-1706.

Fedoruk, J., Schmuland, B., Johnson, J. and Heo, G. 2018, Dimensionality Reduction via the Johnson-Lindenstrauss Lemma: Theoretical Empirical Bounds on Embedding Dimension, Journal of Supercomputing 74:4, 3933-3949.

Geppert, L., Ickstadt, K., Munteanu, A., Quedenfeld, J. and Sohler, C. 2017, Random Projections for Bayesian Regression, Statistics and Computing 27(1), 79-101. 
Ghashami, M., Liberty, E., Phillips, M. and Woodruff, D. 2016, Frequent Directions: Simple and Deterministic Matrix Sketching, SIAM Journal Computing 45(5), 1762-1792.

Heince, C., McWilliams, B. and Meinshausen, N. 2016, Dual Loco: Distributing Statistical Estimation Using Random Projections, 19th International Conference on Artificial Intelligence $\mathbf{5 1}, 875-883$.

Hogben, L. 2007, Handbook of Linear Algebra, Chapman and Hall.

Horvitz, D. and Thompson, D. 1952, A Generalization of Sampling Replacement from a Finite Universe, Journal of the American Statistical Association 47, 663-685.

Indyk, P. and Motwani, R. 1998, Approximate Nearest Neighborhood and the Fast JohnsonLindenstrauss Transform, Proceedings of the 30th ACM Symposium on Theory of Computing pp. 604-613.

Ipsen, I. and Wentworth, T. 2014, The Effect of Coherence on Sampling From Matrices with Orthonormal Columns and Preconditioned Least Squares Problems, Siam Journal of Matrix Analysis and Applicatons 35(4), 1490-1520.

IPUMS 2020, Ruggles, S. and S. Flood and R. Goeken and J. Grover and E. Meyer and J. Pacas and M. Sobek, Vol. Version 10, Minneapolis, p. https://doi.org/10.18128/D010.V10.0.

Johnson, W. and Lindenstauss, J. 1994, Extensions of Lipschitz Maps into a Hilbert Space, Contemporary Mathematics, 26: 189-206.

Jolliffe, I. 1972, Discarding Variables in a Principal Component Analysis: Artificial Data, Applied Statistics 21(2), 160-173.

Kane, D. and Nelson, J. 2014, Sparser Johnson-Lindenstrauss Transforms, Journal of the ACM 61:1, 4.1-4.10.

Li, P., Hastie, T. and Church, K. 2006, Very Sparse Random Projections, KDD pp. 287-296.

Ma, P., Mahoney, M. W. and Yu, B. 2014, A Statistical Perspective on Algorithmic Leveraging, Proceedings of the 31st ICML Conference, Vol. arXiv: 1306.5362.

Madigan, D., Raghavan, N., Dumouchel, W., Nason, M., Posse, C. and Ridgeway, G. 1999, Likelihood-Based Data Squashing: A Modeling Approach to Instance Construction, Technical report, AT and T Labs Ressearch.

Mahoney, M. W. 2011, Randomized Algorithms for Matrices and Data, Foundations and Trends in Machine Learning, http://dx.doi.org/10.1561/2200000035 edn, Vol. 3:2, NOW, pp. 123-224.

Matousek, J. 2008, On Variants of the Johnson-Lindenstrauss Lemma, Random Structure and Algorithms 33(2), 142-156.

McWilliams, B., Krummenacher, C., Lucic, G. and Buhmann, J. 2014, Fast and Robust Least Squares Estimation in Corrupted Linear Models, Advances in Neural Information Processing Systems (NIPS) pp. 415-423.

Meng, X. and Mahoney, M. 2013, Low Distortion Subspace Embeddings in Input-Sparsity time and Applications to Robust Linear Regression, Proceedings of the 45th ACM Symposium on the Theory of Computing.

Mitzenmacher, M. and Upfal, E. 2006, Probability and Computing: Randomized Algorithms and Probabilistic Analysis, Cambridge University Press. 
Nelson, J. and Nguyen, H. 2013a, OSNAP: Faster Numerical Linear Algebra Algorithms via Sparser Subspace Embeddings, Proceedings of the 54th Annual IEEE Symposium on Foundations of Computer Science (FOCS).

Nelson, J. and Nguyen, H. 2013b, Sparsity Lower Bounds for Dimensionality Reducing Maps, STOC pp. 101-110.

Nelson, J. and Nguyen, H. 2014, Lower Bounds for Oblvious Subspace Embeddings, Proceedings of the 41st Interational Colluqium on Automata, Langauges and Programming (ICALP), pp. 883894.

Ng, S. 2017, Opportunities and Challenges: Lessons from Analyzing Terabytes of Scanner Data, in B. Honore, A. Pkes, M. Piazzesi and L. Samuelson (eds), Advances in Economics and Econometrics, Eleventh World Congress of the Econometric Society, Vol. II, Cambridge University Press, pp. $1-34$.

Owen, A. 1990, Empirical Likelihood Ratio Confidence Region, Annals of Statistics 18, 90-120.

Pilanci, M. and Wainwright, M. 2015, Randomized Sketches of Convex Programs with Sharp Guarantees, IEEE Transactions of Information Theory 61(9), 5096-5115.

Pilanci, M. and Wainwright, M. 2016, Iterative Hessian Sketch: and Accurate Solution Approximation for Constrained Least Squares, Journal of Machine Learning Research pp. 1-33.

Portnoy, S. and Koenker, R. 1997, The Gaussian Hare and the Laplacian Tortoise: Computability of Squared-Error versus Absolute-Error Estimation, Statistical Science 12:4, 279-300.

Raskutti, G. and Mahoney, M. 2016, A Statistical Perspective on Randomized Sketching for Ordinary Least Squares, Journal of Machine Learning Research pp. 1-31.

Rudd, P. 2000, An Introduction to Classical Econometric Theory, Oxford University Press.

Sarlos, T. 2006, Improved Approximation Algorithms for Large Matrices via Random Projections, Proceedings of the 47 IEEE Symposium on Foundations of Computer Science, Berkeley, CA, 2006, pp. 143-152.

Vershynin, R. 2017, Four Lectures on Probabilistic Methods for Data Science, arXiv:1612.06661v2.

Wainwright, M. 2019, High-Dimensional Statistics: A Non-Asymptotic Viewpoint, Cambridge Series in Statistical and Probabilistic Mathematics. manuscript in preparation.

Wallace, T. 1972, Weaker Criteria and Tests for Linear Restrictions, Econometrica 40(4), 689-698.

Wang, H., Yang, M. and Stufken, J. 2019, Information-Based Optimal Subdata Selection for Big Data Linear Research, Journal of the American Statistical Association 114:525, 393-405.

Wang, H., Zhu, R. and Ma, P. 2018, Optimal Subsampling for Large Sample Logistic Regression, Journal of the American Statistical Association 113(522), 849-844.

Wang, J., Lee, J., Mahdav, M., Kolar, M. and Srebo, N. 2017, Sketching Meets Random Projection in the Dual: A Provable Recovery Algorithm for Big and High Dimensional Data, Electronic Journal of Statistics 11, 4896-4944.

Wang, S., Gittens, A. and Mahoney, M. 2018, Sketched Ridge Regression: Optimization Perspective, Statistical Perspective, and Model Averaging, Proceedings of the 34th International Conference on Machine Learning 19, 1-50. 
Woodruff, D. 2014, Sketching as a Tool for Numerical Linear Algebra, Foundations and Trends in Theoretical Computer Science 10(1-2), 1-157.

Woolfe, F., Liberty, E., Vladmir, R. and Mark, T. 2008, A Fast Randomized Algorithm for the Approximation of matrices, Applied and Computational Harmonic Analysis 25:3, 335-366.

Yin, Y., Bai, Z. and Krishnaiah, P. 1988, On the Limit of the Largest Eigenvalue of the Largest Dimensional Sample Covariance Matrix, 78:4, 509-521. 